\title{
MODELO PARA ESTIMAÇÃO DA PRODUTIVIDADE DE GRÃOS DE MILHO NO ESTADO DE SÃO PAULO
}

\section{LUÍS GONZAGA MEDEIROS DE FIGUEREDO JÚNIOR}

\begin{abstract}
Tese apresentada à Escola Superior de Agricultura "Luiz de Queiroz", Universidade de São Paulo, para obtenção do título de Doutor em Agronomia, Área de concentração: Irrigação e Drenagem.
\end{abstract}

P I R A C I C A B A

Estado de São Paulo - Brasil

Março - 2004 


\title{
MODELO PARA ESTIMAÇÃO DA PRODUTIVIDADE DE GRÃOS DE MILHO NO ESTADO DE SÃO PAULO
}

\section{LUÍS GONZAGA MEDEIROS DE FIGUEREDO JÚNIOR \\ Engenheiro Agrônomo}

Orientador: Prof. Dr. DURVAL DOURADO NETO

\begin{abstract}
Tese apresentada à Escola Superior de Agricultura "Luiz de Queiroz", Universidade de São Paulo, para obtenção do título de Doutor em Agronomia, Área de concentração: Irrigação e Drenagem.
\end{abstract}

P I R A C I C A B A

Estado de São Paulo - Brasil

Março - 2004 
Dados Internacionais de Catalogação na Publicação (CIP)
DIVISÃO DE BIBLIOTECA E DOCUMENTAÇÃO - ESALQ/USP

Figueredo Júnior, Luís Gonzaga Medeiros de

Modelo para estimação da produtividade de grãos de milho no Estado de São

Paulo / Luís Gonzaga Medeiros de Figueredo Júnior. - - Piracicaba, 2004.

68 p. : il.

Tese (doutorado) - Escola Superior de Agricultura Luiz de Queiroz, 2004.

Bibliografia.

1. Balanço hídrico 2. Desenvolvimento vegetal 3. Época de semeadura 4. Geoprocessamento 5. Grãos 6. Milho 7. Simulação (Estatística) 8. Zoneamento agrícola I. Título

CDD 633.15 
À minha esposa Cleide, pelo carinho e compreensão, e ao filho Luís Neto,

\section{DEDICO}

Aos meus pais e irmãos, pelo apoio e incentivo, 


\title{
DOUTOR DA NOSSA TERRA
}

\author{
Ontem, \\ quando eu só gostava \\ da terra, você, vocês todos \\ sorriram de mim. \\ Hoje, \\ Chamam-me de Doutor. \\ Doutor da minha terra \\ que me enterra, e que \\ enterra quem não é Doutor. \\ Dou todas minhas forças, \\ eu sou minha terra, \\ e quem não é, não é nada. \\ É só terra. \\ E se não a conhece, \\ não será nada não Senhor!
}

Marcos Brito 


\section{AGRADECIMENTOS}

A Deus, por permitir a realização deste curso, o qual só me fez crescer profissional e espiritualmente.

Ao professor Dr. Durval Dourado Neto, pela dedicação e empenho dedicados em sua valiosa orientação.

Ao grande mestre, professor Dr. Nilson Augusto Villa Nova, pelo apoio incondicional na realização do trabalho.

Ao professor Dr. Gerd Sparovek pelo apoio e infra-estrutura disponibilizados para realização do trabalho.

Ao corpo docente ligado ao curso, especialmente aos professores: Dr. Sérgio Nascimento Duarte, Dr. José Antonio Frizzone, Dr. Marcos Vinícius Follegatti, Dr. Tarlei Arriel Botrel, Dr. Quirijn de Jong van Lier e Dr. Antonio Roberto Pereira, pela colaboração durante a realização do curso.

Ao professor Dr. Benito Moreira de Azevedo, pelo incentivo ao ingresso no curso.

Ao professor Dr. Milcíades Gadelha de Lima pela concessão de dados e apoio na realização do trabalho.

Ao professor Dr. Paulo Augusto Manfron, pelas palavras de encorajamento em momentos decisivos.

Ao Engenheiro Agrônomo Alberto Barreto pela contribuição na área de geoprocessamento.

Aos amigos Nildo Dias, Édson Cabral, Edinaldo Mousinho, Marcos Emanuel, René Chipana, Boanerges Júnior e Luiza Duenhas, pela presteza em momentos difíceis. 
Aos colegas de curso Manuel Navarro, Vinícius Maia, Cecília Tojo, Roberta Brushi, Adriana, João Célio, Tales Müller, José Alves Júnior, Tony Araújo e Cláudio Ricardo, pela saudável convivência.

À Universidade de São Paulo, Escola Superior de Agricultura "Luiz de Queiroz", pela oportunidade de realização do curso.

À CAPES, pelo apoio financeiro concedido. 


\section{SUMÁRIO}

Página

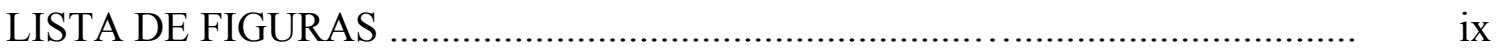

LISTA DE TABELAS ………………………….................................... xi

LISTA DE SÍMBOLOS E ABREVIATURAS ………........................................ xii

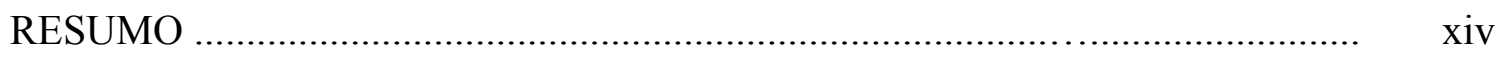

SUMMARY …….......................................................................... xvi

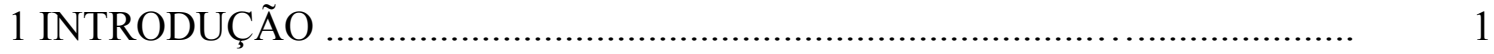

2 REVISÃO DE LITERATURA ……………………..................................

2.1 Cultura de milho: fisiologia da produção ...........................................................

2.2 Elementos do clima e produtividade da cultura de milho ..................................

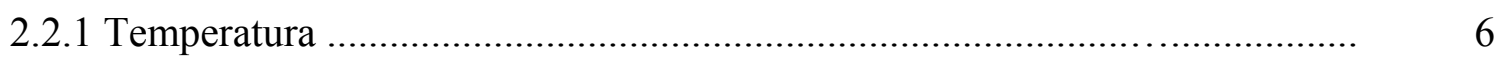

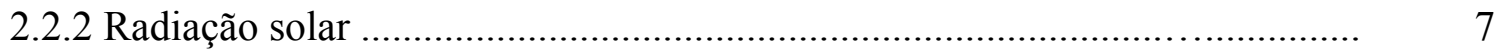

2.2.3 Precipitação pluvial e balanço hídrico ............................................................

2.3 Aptidão agrícola ....................................................................................... 11

2.4 Modelagem em agricultura ………............................................................. 12

2.5 Geoestatística: conceitos e fundamentos gerais .............................................. 15

3 MATERIAL E MÉTODOS _..................................................................... 16

3.1 Desenvolvimento do modelo ……………………...................................... 16

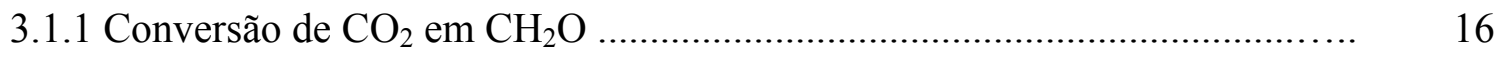

3.1.2 Correção para respiração de manutenção e crescimento $\left(\mathrm{CR}_{\mathrm{MC}}\right)$................ 19

3.1.3 Correção para interceptação de radiação solar (CRs)...................................... 19

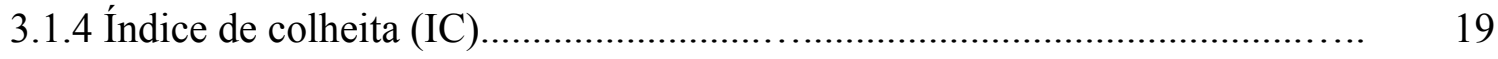

3.1.5 Produtividade potencial de grãos $\left(\mathrm{PP}_{\text {grãos }}\right)$.................................................... 20

3.2 Balanço hídrico........................................................... 20 
3.2.1 Evapotranspiração de referência (ou Potencial)............................. 20

3.2.2 Íncice térmico e coeficiente empírico composto............................ 21

3.2.3 Evapotranspiração máxima.................................................. 21

3.2.4 Capacidade de água disponível........................................ 21

3.2.5 Saldo (S) e negativo acumulado (L) .................................. 22

3.2.6 Variação do armazenamento................................................ 22

3.2.7 Critérios de iniciar o balanço hídrico................................... 22

3.2 .8 Evapotranspiração real............................................... 23

3.2.9 Deficiência hídrica ................................................. 23

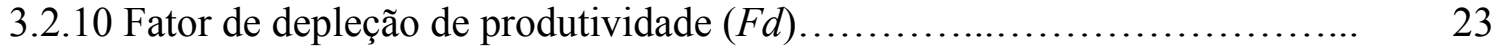

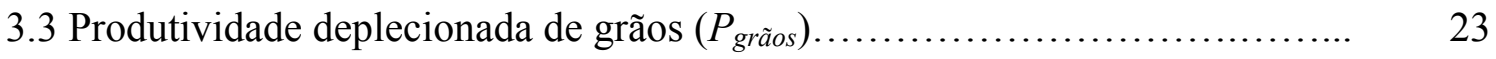

3.4 Avaliação do modelo.................................................. 24

3.5 Análise estatística..................................................... 24

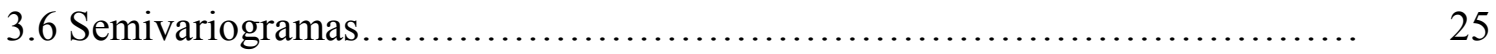

3.7 Elaboração dos mapas.................................................. 26

4 RESULTADOS E DISCUSSÃO.............................................. 29

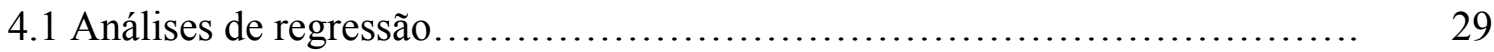

4.2 Variáveis climáticas..................................................... 30

4.3 Estimativas de produtividade potencial de grãos de milho..................... 31

4.4 Deficiência hídrica.................................................. 37

4.5 Estimativas de produtividade deplecionada de grãos de milho................. 38

4.6 Considerações finais...................................................... 57

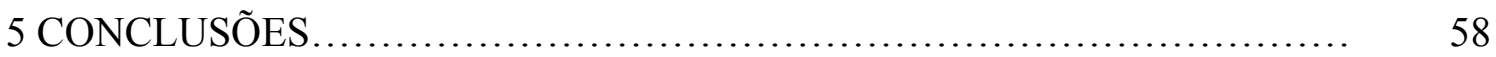

REFERÊNCIAS BIBLIOGRÁFICAS $\quad 59$ 


\section{LISTA DE FIGURAS}

Página

1 Curvas de assimilação de dióxido de carbono (Adc, $\mu \mathrm{L} \mathrm{cm}{ }^{-2} \cdot \mathrm{h}^{-1}$ ) para plantas $\mathrm{C} 4$ em função da radiação solar absorvida (q, cal.cm $\left.{ }^{-2} \cdot \mathrm{min}^{-1}\right)$ e da temperatura $\left(\mathrm{T},{ }^{\circ} \mathrm{C}\right)$ do ar (adaptado de Heemst, 1986)

2 Representação das estações climáticas do Estado de São Paulo com dados de

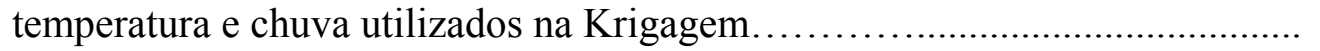

3 Representação dos pontos com dados de radiação solar global utilizados na Krigagem.

4 Temperatura $\left({ }^{\circ} \mathrm{C}\right)$ média dos meses de junho a setembro e dezembro a março no estado de São Paulo

5 Radiação solar global $\left(\right.$ cal.cm $\left.{ }^{-2} \cdot \mathrm{dia}^{-1}\right)$ dos meses de junho a setembro e dezembro a março no Estado de São Paulo

6 Comparação entre produtividades potenciais de grãos de milho observadas $\left(\right.$ Po, kg.ha ${ }^{-1}$ ) (Lima, 1995; Gadioli, 1999; Pioneer, 2002; Forsthofer, 2002) e estimada (Pe, $\left.\mathrm{kg} \cdot \mathrm{ha}^{-1}\right)$ pelo modelo proposto

7 Produtividade potencial $\left(\mathrm{kg} \cdot \mathrm{ha}^{-1}\right)$ de grãos de milho no estado de São Paulo estimada com início de ciclo no primeiro dia dos meses de janeiro, fevereiro, março, abril, maio e junho

8 Produtividade potencial $\left(\mathrm{kg}_{\mathrm{g}} \mathrm{ha}^{-1}\right)$ de grãos de milho no estado de São Paulo estimada com início do ciclo no primeiro dia dos meses de julho, agosto, setembro, outubro, novembro e dezembro

9 Deficiência hídrica $(\mathrm{mm})$ do solo no período referente aos meses de junho a setembro, e dezembro a março no estado de São Paulo 
10 Prodituvidade deplecionada $\left(\mathrm{kg} \cdot \mathrm{ha}^{-1}\right)$ de grãos de milho no estado de São Paulo estimado com época de semeadura no primeiro dia dos meses de janeiro, fevereiro, março, abril, maio e julho................................

11 Produtividade deplecionada $\left(\mathrm{kg} \cdot \mathrm{ha}^{-1}\right)$ de grãos de milho no estado de São Paulo estimado com época de semeadura no primeiro dia dos meses julho, agosto, setembro, outubro, novembro e dezembro.......................... 


\section{LISTA DE TABELAS}

Página

1 Espectro da radiação solar, o seu efeito nas plantas e os instrumentos utilizados na sua medição......................................................................... 8

2 Regiões espectrais de maior importância para as plantas........................ 8

3 Parâmetros referentes aos modelos esféricos ajustados aos semivariogramas dos dados de temperatura utilizados na interpolação por Krigagem.................... 25

4 Parâmetros referentes aos modelos esféricos ajustados aos semivariogramas dos dados de chuva utilizados na interpolação por Krigagem......................... 26

5 Parâmetros referentes aos modelos esféricos ajustados aos semivariogramas dos dados de radiação solar global utilizados na interpolação por Krigagem............. 26

6 Coeficientes e respectivos intervalos de confiança (95\%) das análises de regressão

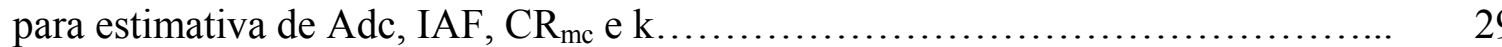

7 Análises de variância das análises de regressão para estimativa de Adc, IAF, CR $_{\mathrm{mc}}$ $\mathrm{e} \mathrm{k}$

8 Produtividades potenciais de grãos de milho observadas $\left(\mathrm{P}_{\mathrm{o}}\right)$ e estimadas $\left(\mathrm{P}_{\mathrm{e}}\right)$ e os

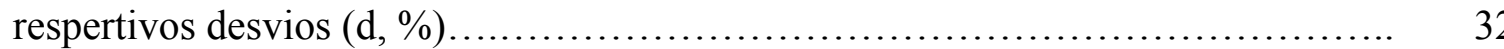

9 Campeões nacionais de produtividade de milho no Brasil no período de 1981 a 1999

10 Genótipos de milho mais recomendados para cada época de semeadura nos 645 municípios do estado de São Paulo. N: normal; P: precoce; S: superprecoce; NR: não recomendável 


\title{
LISTA DE SÍMBOLOS E ABREVIATURAS
}

\author{
$\alpha \quad$ Declinação solar (radianos) \\ $\phi \quad$ Latitude local (radianos) \\ q Radiação solar absorvida (cal.cm $\left.\mathrm{cm}^{-2} \cdot \mathrm{min}^{-1}\right)$ \\ C Duração do ciclo da cultura (dias) \\ $\mathrm{T} \quad$ Temperatura média do ciclo $\left({ }^{\circ} \mathrm{C}\right)$ \\ i Período mensal \\ j Período diário \\ f Período entre a emergência e o florescimento do milho (dias) \\ $\mathrm{H} \quad$ Fotoperíodo (horas) \\ k Coeficiente de extinção da radiação solar \\ u $\quad$ Umidade (\%) \\ Dr Desenvolvimento relativo \\ Fd Fator de depleção de produtividade \\ $\mathrm{T}_{\mathrm{b}} \quad$ Temperatura basal do milho $\left(10^{\circ} \mathrm{C}\right)$ \\ Rs Radiação solar global (cal.cm $\left.{ }^{-2} \cdot \operatorname{dia}^{-1}\right)$ \\ IC Índice de colheita \\ GD Graus-dia ( $\left({ }^{\circ} \mathrm{C}\right.$.dia) \\ $\mathrm{P}_{\mathrm{rc}} \quad$ Poder refletor da cultura \\ $\mathrm{D}_{\mathrm{FR}} \quad$ Duração da fase reprodutiva (dias) \\ $\mathrm{CR}_{\mathrm{s}} \quad$ Correção para interceptação de radiação solar \\ $\mathrm{ET}_{0} \quad$ Evapotranspiração de referência (mm)
}




\begin{tabular}{|c|c|}
\hline I & Índice térmico \\
\hline$a$ & Coeficiente empírico composto \\
\hline $\mathrm{ET}_{\mathrm{m}}$ & Evapotranspiração máxima (mm) \\
\hline $\mathrm{ET}_{\mathrm{r}}$ & Evapotranspiração real (mm) \\
\hline S & Saldo (balanço hídrico) (mm) \\
\hline$L$ & Negativo acumulado (mm) \\
\hline $\mathrm{N}$ & Número de dias para o cálculo de $\mathrm{ET}_{0}$ \\
\hline ppt & Precipitação (ou chuva) (mm) \\
\hline Kc & Coeficiente de cultivo \\
\hline CAD & Capacidade de água disponível (mm) \\
\hline Arm & Armazenamento de água no solo (mm) \\
\hline$\theta_{\mathrm{cc}}$ & Umidade do solo à capacidade de campo $\left(\mathrm{cm}^{3} . \mathrm{cm}^{-3}\right)$ \\
\hline$\theta_{\mathrm{pmp}}$ & Umidade do solo no ponto de murcha permanente $\left(\mathrm{cm}^{3} . \mathrm{cm}^{-3}\right)$ \\
\hline $\mathrm{Ze}$ & Profundidade efetiva do sistema radicular $(\mathrm{cm})$ \\
\hline $\mathrm{Dh}$ & Deficiência hídrica (mm) \\
\hline Exc & Excedente hídrico (mm) \\
\hline $\mathrm{PP}_{\text {grãos }}$ & Produtividade potencial de grãos de milho $\left(\mathrm{kg} \cdot \mathrm{ha}^{-1}\right)$ \\
\hline$P_{\text {grãos }}$ & Produtividade deplecionada de grãos de milho $\left(\mathrm{kg} \cdot \mathrm{ha}^{-1}\right)$ \\
\hline IAF & Índice de área foliar $\left(\mathrm{m}^{2} \cdot \mathrm{m}^{-2}\right)$ \\
\hline Adc & Assimilação de $\mathrm{CO}_{2}\left(\mu 1 . \mathrm{cm}^{-2} \cdot \mathrm{h}^{-1}\right)$ \\
\hline $\mathrm{CR}_{\mathrm{mc}}$ & Correção para respiração de manutenção e crescimento \\
\hline pmp & Ponto de maturidade fisiológica \\
\hline $\mathrm{M}_{\mathrm{CH} 2 \mathrm{O}}$ & Massa bruta de carboidrato produzido $\left(\mathrm{kg} \cdot \mathrm{ha}^{-1}\right)$ \\
\hline
\end{tabular}




\title{
MODELO PARA ESTIMAÇÃO DA PRODUTIVIDADE DE GRÃOS DE MILHO NO ESTADO DE SÃO PAULO
}

\author{
Autor: LUÍS GONZAGA MEDEIROS DE FIGUEREDO JÚNIOR
}

Orientador: Prof. Dr. DURVAL DOURADO NETO

\section{RESUMO}

O presente trabalho tem por objetivo propor um modelo para estimação da produtividade de grãos de milho para o Estado de São Paulo com base nos valores mensais de temperatura, radiação solar e chuva, no intuito de disponibilizar ferramenta para o planejamento regional (identificação das épocas do ano e dos locais mais indicados para o cultivo de milho). O uso de modelos de crescimento e desenvolvimento vegetal possibilita uma economia de tempo, trabalho e quantidade de recursos para tomada de decisões referentes ao manejo no setor agrícola por possibilitar uma previsão do processo de interesse e/ou um melhor entendimento do sistema em estudo. O conhecimento da quantidade de energia solar disponível às plantas, bem como da capacidade de conversão da mesma em energia química metabólica, possibilita prever produtividade de grãos, no caso do milho, quanto à eficiência de conversão em biomassa, através de um modelo geral mecanístico, levando em consideração aspectos agrometeorológicos, fisiológicos, genéticos e edáficos. A assimilação de $\mathrm{CO}_{2}$ pode ser convertida em massa de carboidrato, produzida durante o processo de fotossíntese, em função do índice de área foliar, temperatura e radiação solar absorvida. Estimando-se os valores de radiação solar absorvida, fotoperíodo, índice de área foliar e duração do ciclo, considerando as correções quanto à respiração de manutenção e crescimento, bem como a variação temporal da área foliar, pode-se transformar esse valor em massa líquida de carboidrato total final produzida durante o ciclo. O balanço hídrico foi utilizado com a 
finalidade de estimar a deficiência hídrica durante o ciclo da cultura de milho, sendo adotado o método de Thornthwaite \& Mather (1955), sendo a evapotranspiração de referência estimada pelo método de Thornthwaite (1948). A produtividade deplecionada de grãos de milho foi estimada a partir dos dados de produtividade potencial predita pelo modelo, considerando-se a depleção em função da evapotranspiração relativa (relação entre evapotranspiração real e evapotranspiração da cultura). A partir de dados climáticos obtidos de estações e postos meteorológicos localizados em diversas partes do estado de São Paulo, foram elaborados mapas de superfície no programa TNTmips, com a identificação, por município, das localidades com potencial para o desenvolvimento da cultura de milho. Através destes mapas, é possível identificar restrições quanto à deficiência hídrica, temperatura, radiação solar, produtividade potencial e produtividade de grãos de milho no estado de São Paulo. 


\title{
MODEL TO ESTIMATE CORN YIELD AT SÃO PAULO STATE, BRAZIL
}

\author{
Author: LUÍS GONZAGA MEDEIROS DE FIGUEREDO JÚNIOR \\ Adviser: Dr. DURVAL DOURADO NETO
}

\section{SUMMARY}

The present work has the objective to suggest a model to estimate potential yield and corn yield for São Paulo state based on monthly values of temperature, solar radiation and rainfall, in order to dispose a tool for regional planning (identification of sites and timing during the year more indicated for maize cropping). The use of growing and development models allows savings in time, work and resources needed for making decisions regarding agricultural management, by enabling a preview of the process of interest and/or a better understanding of the system being studied. The knowledge of the available amount of solar energy to plants and the capability of those to convert it into metabolic chemical energy, allows to preview grain yield, in the case of corn, regarding to biomass converting efficiency, through a general mechanistic model, considering agro-meteorological, physiological, genetical and edaphological aspects. The carbon dioxide $\left(\mathrm{CO}_{2}\right)$ assimilation can be converted into mass of carbohydrates produced during photosynthesis process, as a function of leaf area index, air temperature and absorbed solar radiation. By estimating the values of solar radiation, the photoperiod, the leaf area index, and the cycle length, and considering the corrections related to growing and maintenance respiration, as well as the temporal variation of leaf area, it is possible to transform those value into final net mass of total carbohydrate produced during the cycle. The hydric balance, as proposed by Thornthwaite \& Mather (1955), was used with the objective to estimate the hydric deficit during maize cycle, being the reference 
evapotranspiration being estimated by Thornthwaite (1948). Corn yield was estimated up from potential yield predicted by the model, considering the depletion from the relative evapotranspiration (relation between real evapotranspiration and crop evapotranspiration). Starting from climatic data obtained from several meteorological station located at different sites in São Paulo State, Brazil, surface maps were elaborated using TNTmips software, with the identification, by county, of those sites with maize development potential. Through those maps it is possible to identify restriction regarding hydric deficit, temperature, solar radiation, potential yield, and corn yield in São Paulo State. 


\section{INTRODUÇÃO}

Em função do valor nutritivo e dos altos rendimentos alcançados, o milho (Zea mays L.) é um dos cereais mais cultivados no mundo, assumindo grande importância social e econômica. No Brasil, o milho é cultivado em todas as regiões, ocupando uma área de aproximadamente 13 milhões de hectares (Conab, 2004a).

A produtividade média de grãos de milho (safra normal) no Brasil e no Estado de

São Paulo é da ordem de 3.500 kg.ha ${ }^{-1}$ e 4.600 kg.ha ${ }^{-1}$, respectivamente (Conab, 2004b), valores muito abaixo do desejado, considerando seu potencial produtivo, que com a utilização de alto nível tecnológico, ultrapassa $16.000 \mathrm{~kg} \cdot \mathrm{ha}^{-1}$ (Coelho, 2003). Todavia, a cultura de milho vem experimentando importantes avanços nos mais diversos campos da ciência agronômica, possibilitando uma melhor compreensão do processo produtivo e de suas interações com o meio, o que tem gerado aumentos significativos na produtividade, principalmente na região Centro-Sul, responsável por mais de $90 \%$ do total produzido no país (Conab, 2004a).

Novas tecnologias como sistemas de informações geográficas, sensoriamento remoto e modelos de simulação de crescimento, desenvolvimento e produtividade de culturas, vêm sendo desenvolvidas nas últimas décadas, abrindo novos caminhos para pesquisa de planejamento e manejo de práticas agrícolas.

O uso de modelos de culturas para simulações em computadores possibilita uma economia de tempo, trabalho e quantidade de recursos para planejamento e tomada de decisões de manejo no setor agrícola. A utilização de modelos de culturas em associação a técnicas de geoprocessamento facilita a identificação de locais e épocas mais recomendadas para o desenvolvimento das culturas, tornando-se uma importante ferramenta para o zoneamento agrícola e difusão de informações. 
O conhecimento das relações energia-planta é imprescindível para a elaboração de modelos de cultura bem estruturados. Dessa maneira, para que os resultados obtidos sejam satisfatórios, os principais processos fisiológicos da planta, bem como sua interação com as condições do ambiente, devem ser corretamente estimados. Não obstante, a caracterização espaço-temporal de elementos climáticos como temperatura, radiação solar e chuva, é um fator decisivo para a aplicação de um modelo de cultura.

O presente trabalho tem por objetivo propor um modelo para estimação da produtividade de grãos de milho para o Estado de São Paulo com base nos valores mensais de temperatura, radiação solar e chuva, no intuito de disponibilizar ferramenta para o planejamento regional (identificação dos locais e das épocas do ano mais indicados para o cultivo de milho). 


\section{REVISÃO DE LITERATURA}

\subsection{Cultura de milho: fisiologia da produção}

Entre as principais culturas de cereais dos trópicos sub-úmidos e semi-áridos, o milho tem a mais alta produtividade em condições adequadas de água e fertilidade do solo. Porém, é uma das culturas mais sensíveis ao estresse hídrico (Lima, 1995). Além disso, a capacidade da planta em produzir fitomassa seca está diretamente relacionada com a quantidade de energia luminosa disponível e com a capacidade de aproveitamento dessa energia. Por esse motivo, torna-se importante a análise do desenvolvimento da cultura em diferentes situações (Costa, 1994).

O milho, por ser um planta tipo $\mathrm{C} 4$, apresenta características fisiológicas favoráveis no que se refere à eficiência de conversão de carbono mineral (representado pelo gás carbônico da atmosfera) em compostos orgânicos como os carboidratos. Isto ocorre porque no processo fotossintético destas plantas, o $\mathrm{CO}_{2}$ é continuamente concentrado nas células da bainha vascular das folhas e, em seguida, redistribuído para posterior utilização (Salisbury, 1992).

A principal diferença fisiológica entre as plantas que apresentam fotossíntese C3 e C4, está relacionada ao processo de fotorrespiração. As plantas de fotossíntese C3 perdem de 20 a $50 \%$ do carbono fixado devido a fotorrespiração, enquanto as plantas com fotossíntese $\mathrm{C} 4$, como o milho, não apresentam perdas mensuráveis de $\mathrm{CO}_{2}$ neste processo (Floss, 2002).

A respiração é o processo pelo qual compostos altamente energéticos são sintetizados a partir dos carboidratos produzidos na fotossíntese, podendo ser dividida, teoricamente, em respiração de crescimento e de manutenção. A respiração de crescimento é definida como a produção de novos compostos orgânicos, enquanto a de 
manutenção consiste na realocação de outros compostos existentes e está relacionada com o consumo de energia para promover a organização estrutural da planta (Oliveira, 1990).

O rendimento fotossintético da planta representa a interação entre a resposta fotossintética de folhas individuais com a disponibilidade de luz que incide sobre elas (Costa, 1994). A eficiência de interceptação de radiação solar depende da idade da planta, da arquitetura foliar, do arranjo espacial de plantas e da população empregada, ao passo que a eficiência de conversão, depende principalmente da temperatura, do estado nutricional e do equilíbrio hídrico das plantas.

A fotossíntese é função da magnitude da área foliar e da duração das folhas em plena atividade com balanço energético positivo, enquanto a variação temporal da área foliar depende do tipo de cultura. Geralmente, a área foliar aumenta até um máximo, onde permanece por algum tempo, decrescendo em seguida, sobretudo em função da senescência das folhas mais velhas (Pereira \& Machado, 1987). Nesse sentido, Gadioli (1999) acrescenta que a produtividade da cultura será maior quanto mais rápido a planta atingir o índice de área foliar máximo e quanto mais tempo a área foliar permanecer ativa.

A partição dos fotoassimilados é função principalmente do genótipo e das relações de fonte-dreno (Dourado Neto, 1999). O índice de colheita, ou seja, a fração dos grãos produzidos em relação à fitomassa seca total da planta pode indicar a habilidade de um híbrido combinar produtividade e destinar fitomassa seca acumulada aos componentes de interesse econômico (Fancelli \& Dourado Neto, 2000).

\subsection{Elementos do clima e produtividade da cultura de milho}

A importância das condições climáticas, durante a estação de crescimento na produtividade da cultura de milho, é amplamente reconhecida por muitos pesquisadores (Fancelli \& Lima, 1982; Rosenberg et al., 1983; Cruz et al., 1994; Dourado Neto, 1999; Fancelli \& Dourado Neto, 2000). Por outro lado, as características agroclimáticas de várias localidades podem influenciar diferentemente a produtividade da cultura. 
Entende-se por produtividade potencial de uma cultura, a máxima produção por unidade de área obtida por um genótipo altamente produtivo e bem adaptado ao respectivo ambiente, em condições (durante todo o ciclo) de adequado suprimento de água e nutrientes, e sem sofrer limitações por fatores externos tais como pragas e doenças. A produtividade deplecionada da cultura, por sua vez, é a produção por unidade de área em condições tais que pelo menos um fator interfere no processo produtivo, limitando o potencial do genótipo considerado (Doorenbos \& Kassam, 1994).

Dessa maneira, a extensão com que a cultura pode expressar o seu potencial genético é determinada por sua interação com a radiação solar, temperatura do ar, pressão de vapor d'água na atmosfera, velocidade do vento e características físicohídricas do solo (Rosenberg et al., 1983). A quantificação da relação entre a produtividade da cultura e variáveis agroclimáticas, permite que o impacto dessas variáveis na produtividade, durante o ciclo da cultura, seja avaliado (Mondragón, 1990).

O milho, sendo uma planta de origem tropical, exige, durante o seu ciclo vegetativo, calor e água para se desenvolver e produzir satisfatoriamente, proporcionando produtividades compensadoras. Independente da tecnologia aplicada, o período de tempo e as condições climáticas em que a cultura é submetida constituem-se em preponderantes fatores de produção.

A quantificação dos elementos do clima, portanto, pode ser utilizada no ajuste de modelos de simulação de desenvolvimento e crescimento de culturas, tornando-se um importante instrumento para pesquisa, planejamento e monitoramento de culturas (Pandolfo, 1995).

A utilização de modelos probabilísticos adequados para estimativa da distribuição de chuva, radiação solar e temperatura, permite obter precisão matemática e resultados consistentes, mesmo em séries históricas mais curtas de dados (Camargo et al., 1985; Frizzone et al., 1985; Alfonsi et al., 1995). Entretanto, em alguns casos não é possível dispor de séries históricas de dados climáticos. Nessas circunstâncias, a média passa a ser o parâmetro utilizado. 


\subsubsection{Temperatura}

A temperatura expressa, em valores numéricos, o nível de energia interna em função da temperatura do ar naquele momento, possibilitando trocas com o sistema e o meio, provocando estímulos, ativando ou desativando funções vitais, tais como a respiração e transpiração (Ometto, 1981).

A elevação da temperatura contribui para a redução da taxa fotossintética líquida em função do aumento da respiração, interferindo diretamente na produção. Assim, temperaturas elevadas prevalecentes no período noturno (superior a $24^{\circ} \mathrm{C}$ ) promovem um consumo energético demasiado, em função do incremento da respiração celular, ocasionando menor saldo de fotoassimilados, com conseqüente queda na produtividade da cultura de milho (Dourado Neto, 1999). Em contrapartida, a maioria dos materiais (híbridos ou variedades cultivadas) existentes atualmente não se desenvolve em temperaturas inferiores a $10{ }^{\circ} \mathrm{C}$, que é considerada a temperatura basal para a espécie (Villa Nova et al., 1972).

No desenvolvimento do milho, a duração do ciclo em dias tem demonstrado inconsistência. Isso se deve ao fato de que a duração de subperíodos e dos ciclos da planta estão associados às variações das condições ambientais, e não ao número de dias dos meses. De forma generalizada, a temperatura apresenta-se como o elemento climático mais importante para predizer os eventos fenológicos da cultura (Gadioli, 1999).

Tendo em vista o sucesso na predição de datas de ocorrência dos estádios de desenvolvimento da cultura do milho, modeladores têm assumido que o conceito unidade térmica é universalmente aplicável (Lima, 1995). Conforme Villa Nova et al. (1972), a quantidade de energia exigida por uma cultura tem sido expressa em graus-dia, ou unidades térmicas de desenvolvimento, exigência térmica, exigência calórica. A base teórica para essa técnica é que, dos processos envolvidos no desenvolvimento da cultura, todos são sensíveis à temperatura do ar, cabendo enfatizar que a resposta das plantas à temperatura do ar obedece a limites (inferior e superior) e é extensiva ao desenvolvimento total da cultura. 
Os genótipos de milho são classificados comercialmente em normais, precoces e superprecoces, conforme suas exigências calóricas no período compreendido entre a emergência e o florescimento (fase vegetativa). Os genótipos normais apresentam exigências térmicas de 890 a $1200^{\circ} \mathrm{C}$.dia, os precoces, de 831 a $890^{\circ} \mathrm{C}$.dia, e os superprecoces, de 780 a $830^{\circ} \mathrm{C}$.dia (Embrapa, 2004).

\subsubsection{Radiação solar}

A radiação solar que alcança a superfície terrestre pode ser separada basicamente em dois componentes, um quando a radiação é direta, outro quando é refletida ou espalhada por nuvens e pela atmosfera (Goudriaan \& Laar, 1992). A radiação independente de ser direta ou difusa pode ser separada em duas bandas, ou seja, radiação de baixo comprimento de onda $(<3000 \mathrm{~nm})$ e radiação térmica $(>3000 \mathrm{~nm})$.

As radiações de baixo comprimento de onda são subdivididas ainda em bandas, as quais produzem diferentes efeitos sobre as plantas (Tabela 1 e Tabela 2). A faixa de radiação dita visível (400 a $700 \mathrm{~nm}$ ) tem especial interesse porque estas radiações são fotossinteticamente ativas (Kubins, 1971).

O balanço de energia radiante, denominado de radiação líquida, vem a ser o saldo de radiação sobre uma superfície (Ometto, 1981). Algumas considerações sobre a importância do balanço de energia e a radiação líquida na determinação do fluxo de vapor d'água na atmosfera são feitas por Villanueva (1987) e diversos foram os estudos desenvolvidos por pesquisadores voltados ao monitoramento do saldo de radiação, bem como aos aspectos de sua partição nos mais variados sistemas agrícolas. Esses fatores são de grande importância para verificação da quantidade e distribuição de luz na cobertura vegetal.

A radiação solar é praticamente a única fonte de energia para os processos fisiológicos e bioquímicos que ocorrem nos vegetais. Sendo assim, a produção final de fitomassa seca de uma planta depende, em última instância, da eficiência com que as folhas convertem energia radiante em energia química por meio da fotossíntese (Assis \& Mendez, 1989). 
Tabela 1. Espectro da radiação solar, o seu efeito nas plantas e os instrumentos utilizados na sua medição

\begin{tabular}{cccc}
\hline Nome & Faixa $(\mathrm{nm})$ & Efeitos nas plantas & Instrumentos \\
\hline Ultravioleta & $<280$ & Morte rápida & Prejudicial \\
& $280-315$ & Morfogênico & Termófilo com filtros espectrais \\
Visível & $415-400$ & Fotossintético & Medidor de fluxo quântico \\
Vermelho & $610-700$ & Morfogênico & Termófilo com filtros \\
Infravermelho & $700-800$ & Morfogênico & Termófilo com filtros \\
Ondas-curtas & $300-3000$ & $\begin{array}{c}\text { Fotossintético, } \\
\text { Morfogênico, balanço } \\
\text { energético }\end{array}$ & Termófilo coberto com vidro \\
$\begin{array}{c}\text { Terrestre } \\
\text { (térmica) }\end{array}$ & $3 \times 10^{3}-10^{5}$ & Balanço energético & Termófilo com filtros \\
$\begin{array}{c}\text { Radiação total e } \\
\text { líquida }\end{array}$ & $300-10^{5}$ & Balanço energético e & Termófilos com polietileno \\
\hline
\end{tabular}

Fonte: Kubins (1971)

Tabela 2. Regiões espectrais de maior importância para as plantas

\begin{tabular}{|c|c|c|}
\hline Região espectral (nm) & Caráter de absorção & Efeito fisiológico \\
\hline \multicolumn{3}{|l|}{ Ultravioleta } \\
\hline$<280$ & Pelo protoplasma & Letal em grandes quantidades \\
\hline $280-315$ & Pelo protoplasma & $\begin{array}{l}\text { Grande efeito morfogênico; estimula } \\
\text { algumas biossínteses; grande efeito nos } \\
\text { processos fisiológicos }\end{array}$ \\
\hline $315-400$ & $\begin{array}{l}\text { Pelas clorofilas e } \\
\text { protoplasma }\end{array}$ & $\begin{array}{l}\text { Sem nenhum efeito específico, pequeno } \\
\text { efeito na fotossíntese }\end{array}$ \\
\hline \multicolumn{3}{|l|}{$\begin{array}{c}\text { Radiação ativa } \\
\text { fotossinteticamente }\end{array}$} \\
\hline $400-510$ & $\begin{array}{c}\text { Forte, pelas clorofilas e } \\
\text { carotenóides }\end{array}$ & $\begin{array}{l}\text { Grande efeito na fotossíntese; grande } \\
\text { efeito morfogênico }\end{array}$ \\
\hline $510-610$ & Menos que o anterior & Pequeno efeito na fotossíntese \\
\hline $610-720$ & Forte, pelas clorofilas & Grande efeito na fotossíntese \\
\hline \multicolumn{3}{|l|}{ Infravermelho } \\
\hline $720-1000$ & Fraca & Estimula o processo de elongação \\
\hline$>1000$ & Pela água dos tecidos & $\begin{array}{c}\text { Sem nenhum efeito sobre os processos } \\
\text { fotoquímicos ou bioquímicos; } \\
\text { convertido a calor }\end{array}$ \\
\hline
\end{tabular}

Fonte: Kubins (1971) 
Com relação ao fotoperíodo, o milho é considerado como planta neutra ou de dias curtos (Reichardt, 1987; Doorenbos \& Kassam, 1994). Seu desenvolvimento é, porém, muito afetado pela quantidade de radiação solar, e as maiores produtividades são alcançadas em condições de altas radiações, em virtude de pertencer ao grupo de plantas “C4”, o que lhe confere alta produtividade biológica (Fancelli \& Dourado Neto, 2000).

\subsubsection{Precipitação pluvial e balanço hídrico}

Nas regiões tropicais, a precipitação pluvial ou chuva, é a forma principal pela qual a água retorna da atmosfera para a superfície terrestre após os processos de evaporação e condensação, completando, assim, o ciclo hidrológico. A quantidade e a distribuição de chuvas que ocorrem anualmente em uma região determinam o tipo de vegetação natural e o tipo de exploração agrícola possível (Pereira et al., 2002).

A freqüência de chuvas é sem dúvida um dos elementos climáticos mais importantes que afetam a produtividade de determinada cultura (Alfonsi et al., 1995). Dessa maneira, o conhecimento da probabilidade de ocorrência de chuvas é importante para manejo das culturas e planejamento da irrigação (Cruz et al., 1994; Alfonsi et al., 1997). Para tanto, é necessário dispor de uma série de dados de chuva no local ou região. Um método simples de calcular a probabilidade de ocorrência de chuva a partir de uma seqüência de valores medidos, segundo Thom (1966), baseia-se na ordenação crescente ou decrescente de valores. Se a ordenação for crescente, a probabilidade corresponderá a um valor igual ou menor que o limite escolhido; se for decrescente, estima-se o inverso.

A quantidade de água que se encontra disponível no solo durante as fases fenológicas da cultura é fundamental para o sucesso da produção agrícola. Dessa maneira, Fancelli \& Dourado Neto (2000) justificam que a necessidade de suprimento hídrico para o pleno desenvolvimento dos vegetais, decorre das múltiplas funções que ela desempenha na fisiologia das plantas, pois, praticamente todos os processos metabólicos são influenciados pelo conteúdo de água.

Os processos que envolvem fluxo de água (infiltração, redistribuição, evaporação e absorção pelas plantas) são interdependentes e, quase sempre, ocorrem simultaneamente. Reichardt \& Timm (2004) consideram que para estudar o ciclo da 
água em um solo vegetado, é necessário considerar o balanço hídrico, que se refere ao somatório das quantidades de água que entram e saem de um volume de solo, num dado intervalo de tempo, sendo o resultado a quantidade líquida de água que nele permanece disponível às plantas.

O balanço hídrico é estimado com a finalidade de se conhecer deficiência e/ou excedente hídricos durante o ciclo de uma cultura, constituindo-se uma ferramenta muito útil para a recomendação ou não do seu cultivo em determinada região, bem como para definir época de semeadura (Thornthwaite \& Mather, 1955). Assim, um balanço entre a precipitação e a evapotranspiração, pode indicar de forma mais consistente as disponibilidades hídricas de uma região no decorrer do ano, auxiliando sobremaneira no planejamento efetivo da cultura em função do local escolhido, bem como sobre a necessidade do uso de irrigação em determinada época do ano (Fancelli \& Lima, 1982).

Na elaboração do balanço hídrico climatológico, o primeiro passo é a seleção da capacidade de água disponível - CAD, ou seja, a lâmina de água correspondente ao intervalo de umidade entre a capacidade de campo - CC e o ponto de murcha permanente - PMP (Pereira et al., 2002). Como o balanço hídrico é mais utilizado para fins de caracterização da disponibilidade hídrica de uma região em bases climatológicas e comparativas, a seleção da CAD é feita em função do tipo de cultura ao qual se quer aplicá-lo e do tipo de solo (Thornthwaite \& Mather, 1955).

Para suprir as exigências hídricas da cultura do milho, segundo Fancelli \& Lima (1982), deve ocorrer um mínimo de $200 \mathrm{~mm}$ de precipitação no verão, sem a necessidade de irrigação. Reichardt (1987) comenta que genótipos de ciclo normal exigem de 500 e $800 \mathrm{~mm}$ de água por ciclo. Entretanto, Fancelli \& Dourado Neto (2000), destacam que tal limitação encontra-se mais diretamente condicionada à distribuição, do que à quantidade total de chuvas ocorrida no período.

O consumo de água pela cultura do milho varia de acordo com a localização geográfica e condições climáticas. Segundo Castro (1979) foi observado um consumo médio de 4,7 mm por dia em Piracicaba - SP, enquanto Saunders et al. (1984), obtiveram um consumo médio de 5,8 $\mathrm{mm}$ por dia no município de Quixadá - CE. Outras observações experimentais referentes a essa cultura, evidenciam índices diários de 
consumo de água próximos a $10 \mathrm{~mm}$, quando as plantas encontram-se submetidas a condições de intenso calor e baixa umidade relativa do ar (Daker, 1969).

A cultura do milho apresenta três estádios de desenvolvimento mais sensíveis ao déficit hídrico: o primeiro é a iniciação floral e desenvolvimento da inflorescência, quando o número potencial de grãos é determinado; o segundo refere-se ao subperíodo de fertilização, quando o potencial de produção é estabelecido; o terceiro é o do enchimento de grãos (Paterniani, 1978). Por outro lado, as fases mais sensíveis ao excedente hídrico são a germinação, a diferenciação do pendão e, do espigamento até a maturação fisiológica (Mondragón, 1990).

A relação entre a produtividade relativa e a evapotranspiração relativa (razão entre a evapotranspiração real e a evapotranspiração máxima) vem sendo utilizada em modelos de simulação de culturas que utilizam disponibilidades energética (Doorenbos \& Kassam, 1994) e hídrica (Chang, 1968, citado por Medeiros et al., 1991), assumindo grande importância no planejamento das culturas irrigadas. Uma vez estabelecida a curva de resposta entre evapotranspiração relativa e produtividade, é possível avaliar a eficiência da irrigação sobre o rendimento, o que permite obter uma estimativa com e sem o uso da irrigação, a partir de dados do balanço hídrico.

\subsection{Aptidão agrícola}

Uma região apresenta várias sub-regiões com distintas condições de solo e clima e, portanto, com potenciais distintos para a exploração econômica de determinada cultura (Ramalho Filho \& Beek, 1995).

A aptidão agrícola dos solos para a agricultura, segundo Fancelli \& Lima (1982), é influenciada por quatro fatores distintos e diretamente ligados a uma atividade agrícola racional, ou seja: (i) fertilidade; (ii) disponibilidade de água; (iii) susceptibilidade à mecanização e (iv) deficiência de aeração. O melhoramento de quaisquer desses fatores, por sua vez poderá ser extremamente dependente do grau de conhecimento técnico do agricultor, das condições sócio-econômicas predominantes na região, do complexo climático local, das oportunidades de mercado, do destino da produção, além de outros relativos à situação reinante no momento. 
Fancelli \& Dourado Neto (2000) acrescentam que para o milho, bem como para qualquer outra cultura, evidencia-se a necessidade da ocorrência de um conjunto de fatores edafoclimáticos plenamente interligados, de forma a propiciar um desenvolvimento adequado à planta, conferindo rendimento compensatório.

A elaboração de modelos eficientes que permitem obter a previsão de safra e a indicação da cultura de milho para uma determinada região está na dependência do conhecimento das exigências calóricas dessa cultura e das condições dos elementos do clima (radiação solar, temperatura e precipitação pluvial, principalmente), bem como da correta interpretação de levantamento de solos.

\subsection{Modelagem em agricultura}

Reynolds (1979) definiu modelo como sendo uma equação ou conjunto de equações que representam um processo. Para De Wit (1982), modelo é a representação simplificada de um sistema, enquanto simulação é a arte de construir modelos matemáticos. Em ciência, modelos são ferramentas que ajudam a conceituar, integrar e generalizar o conhecimento científico (Leal \& De-Polli, 1999).

Existem diversas classificações propostas para diferenciar os modelos. Uma delas divide os modelos em matemáticos e de simulação. O primeiro tipo refere-se a representações matemáticas de um fenômeno, enquanto o segundo engloba um ou mais modelos matemáticos, representando fenômenos mais complexos (Barros, 1998).

Os modelos matemáticos podem ser de três tipos: (i) empíricos, baseados em dados puramente observados; (ii) estocásticos, em que o processo é descrito pelas leis de probabilidade e (iii) mecanísticos, que consideram as leis físicas, químicas e biológicas no processo, sendo os mais versáteis dentre os tipos de modelos matemáticos (Pautian et al., 1992).

Os modelos de simulação, conforme Addiscott (1993) podem ser divididos em: (i) determinísticos, em que um conjunto de eventos leva a um resultado único e definido e (ii) estocásticos, em que a incerteza é considerada na sua estrutura. Essas duas categorias mencionadas podem ainda dividir os modelos de simulação em: (i) 
mecanísticos, que procuram descrever os mecanismos envolvidos no processo e (ii) funcionais, que descrevem apenas os aspectos gerais do processo.

Pesquisadores de diversas partes do mundo vêm usando vários modelos de culturas, testando a habilidade na simulação de eventos fenológicos, produção de biomassa e produtividade de grãos (Dourado Neto, 1999).

Segundo Thornley (1976), inúmeras vantagens podem ser conseguidas com o uso de modelos bem elaborados, considerando que os mesmos devem resumir convenientemente uma gama de informações, permitindo progressos no conhecimento da planta e suas interações com o ambiente, além de esclarecer pontos em que o conhecimento seja limitado.

Os modelos de simulação de crescimento e previsão de produtividade de culturas permitem fazer simulações de longo prazo, sendo realizadas geralmente a um baixo custo, utilizando-se características do solo e práticas de manejo da cultura durante o período de dados climatológicos históricos disponíveis para determinado local (Muchov et al., 1991).

A previsão de produtividade torna-se mais precisa quando os modelos de simulação são usados para estimar a produção em grandes áreas (Lozarda \& Angelocci, 1999). Por outro lado, Hoogenboom (2000) afirma que a utilização de modelos, com fins de predição, pode ter aplicações, tanto previamente à semeadura, como durante o crescimento e desenvolvimento da cultura, podendo essa informação ser usada ao nível de propriedades rurais ou de instituições governamentais para planejamento de políticas agrícolas.

Diversos autores desenvolveram modelos que simulam o desenvolvimento da planta, o acúmulo de fitomassa seca nos diferentes componentes da planta e o índice de área foliar, em função de parâmetros fenológicos e climáticos (Keulen et al., 1982; Keulen \& Wolf, 1986; Spitters et al., 1986a,b). Cada um destes modelos simula parte do processo de produção da planta ou cultura, sendo desenvolvidos em condições climáticas específicas. Apesar de sua importância para a pesquisa e a agricultura, sua aplicação pode tornar-se limitada quando se pretende fazer estimativas em condições distintas daquelas em que tais modelos foram desenvolvidos. 
O modelo "Water and Agrochemical Vadose Environment" (WAVE) foi desenvolvido em condições climáticas mais amplas. Esse modelo é parte de um projeto do Instituto de Pesquisas científicas na agricultura e indústria da Bélgica, sendo resultado de inúmeras pesquisas que objetivaram o desenvolvimento, a calibração e validação de modelos matemáticos que descrevem a quantidade de matéria e energia no sistema solo-planta-atmosfera, e consistem de diferentes módulos de simulação do crescimento de plantas e movimento da água, solutos, calor e nitrogênio no solo (Vanclooster et al., 1994).

Uma equipe interdisciplinar do Departamento de Agricultura dos Estados Unidos desenvolveu o modelo Crop-Environment Resource Synthesis (CERES)-Maize com o objetivo de quantificar os efeitos independentes e interativos do genótipo, clima e atributos do solo no crescimento e produção de milho. O referido modelo simula os principais processos fisiológicos da planta, incluindo: fotossíntese; respiração; acumulação e partição de fitomassa; fenologia; crescimento de folhas, caules e raízes; extração de água do solo e, evapotranspiração (Hoogenboom, 2000).

Os modelos WAVE e CERES-Maize, são capazes de simular satisfatoriamente em distintas situações (Vanclooster et al., 1994, Lima, 1995; Soler, 2000). Contudo, estes modelos são bastante complexos e sua aplicação requer uma grande quantidade de parâmetros de entrada, tornando sua utilização limitada pela dificuldade na obtenção dos dados requeridos nas simulações.

Com base nas relações energia-planta, um procedimento mais simples foi desenvolvido por Doorenbos \& Kassam (1994) para estimativa da produtividade potencial de diversas culturas, incluindo o milho. Simulações realizadas com a utilização deste modelo, requerem uma quantidade relativamente pequena de dados de entrada, o que facilita sua aplicação (Villa Nova et al., 2001). O modelo desenvolvido no presente trabalho tem por base as equações apresentadas por Doorenbos \& Kassam (1994) para estimativa da produtividade potencial da cultura de milho. 


\subsection{Geoestatística: conceitos e fundamentos gerais}

A estatística clássica utiliza parâmetros como média e desvio padrão para representar um fenômeno, e baseia-se na hipótese de que as variações de um local para outro são aleatórias (Gomes \& Garcia, 2002). Quando uma determinada propriedade varia de um local para outro com algum grau de organização ou continuidade, expresso pela dependência espacial, deve-se utilizar um enfoque relativamente novo, ou seja, a geoestatística (Oliver \& Webster, 1991; Gaston et al., 2001). Este enfoque tem como base, a teoria das variáveis regionalizadas, que é a aplicação do formalismo das funções aleatórias para o reconhecimento e a estimativa de fenômenos naturais (Matheron, 1971).

Para determinar qual dessas duas áreas da estatística é mais adequada para cada caso, a ferramenta utilizada é o semivariograma, que expressa a dependência espacial entre as amostras (Gonçalves, 1997; Frogbrook et al., 2002). O ajuste de modelos matemáticos aos pontos de um semivariograma, segundo Goovaerts (1999), normalmente é feito pelos métodos da minimização do quadrado dos desvios, assumindo normalidade e independência dos resíduos e homogeneidade de variâncias.

Para Webster (1985), um modelo matemático a ser ajustado ao semivariograma, necessita incluir três parâmetros: um intercepto ou "efeito pepita", um "patamar", atingido após uma distância correspondente ao "alcance". Além disto, a forma da curva deve se ajustar aos pontos experimentais na região de crescimento da função, ou seja, para a distância entre zero e o alcance.

No caso de haver dependência espacial, pode-se estimar valores da propriedade em estudo para os locais não amostrados na área considerada, por meio de técnicas de interpolação. O método da krigagem, bastante utilizado em ciência agronômica (Goovaerts, 1999; Viana da Mata et al., 1999; Deeggle \& Ribeiro Júnior, 2000; Cerri, 2003), utiliza um interpolador não tendencioso e de variância mínima que assegura a melhor estimativa dos dados, por ser de variância mínima e por assegurar que o somatório dos pesos é igual à unidade. Essa estimativa tem como base os dados amostrais da variável regionalizada e as propriedades estruturais do semivariograma obtido a partir destes dados (Gonçalves, 1997). 


\section{MATERIAL E MÉTODOS}

\subsection{Desenvolvimento do modelo}

Baseado nas relações entre dados agroclimáticos e a conversão de energia solar que resulta em produção de fitomassa seca, elaborou-se o modelo para estimar a produtividade da cultura de milho.

\subsubsection{Conversão de $\mathrm{CO}_{2}$ em $\mathrm{CH}_{2} \mathrm{O}$}

A fixação de dióxido de carbono $\left(\mathrm{CO}_{2}\right)$ pelas plantas, para a produção bruta de carboidrato $\left(\mathrm{CH}_{2} \mathrm{O}\right)$ está relacionada com a fração da radiação fotossinteticamente ativa do espectro solar, de acordo com a seguinte equação:

$$
\mathrm{CO}_{2}+\mathrm{H}_{2} \mathrm{O}+\text { energia solar } \rightarrow \mathrm{CH}_{2} \mathrm{O}+\mathrm{O}_{2}
$$

A assimilação de $\mathrm{CO}_{2}$ pelas plantas $\mathrm{C} 4$ praticamente cessa com baixos valores de energia, e varia também em função da temperatura. A relação de dependência entre a fixação de $\mathrm{CO}_{2}$ pela cultura de milho, radiação solar absorvível e temperatura (Figura 1), foi construída com base em dados experimentais obtidos por Heemst (1986), que quantificou o processo em câmaras de controle interno de temperatura e irradiância. A partir destes dados chegou-se ao modelo para estimativa da assimilação de $\mathrm{CO}_{2}$ em plantas C4, expresso pela equação:

$$
A d c=a \cdot e^{-\frac{1}{2}\left\{\frac{1}{c}\left[\ln \left(\frac{q}{b}\right)\right]^{2}+\frac{1}{f}\left[\ln \left(\frac{T}{d}\right)\right]^{2}\right\}}
$$

em que $A d c$ se refere à assimilação de $\mathrm{CO}_{2}\left(\mu \mathrm{L} \cdot \mathrm{cm}^{-2} \cdot \mathrm{h}^{-1}\right), q$ à radiação solar absorvida $\left(\right.$ cal.cm $\left.{ }^{-2} \cdot \min ^{-1}, 0<q \leq 0,4\right), T$ à temperatura média do ciclo $\left({ }^{\circ} \mathrm{C}, 15 \leq T \leq 35\right)$, e $a, b, c$, $d, f$ aos parâmetros empíricos determinados através de análise de regressão múltipla 
$\left(a=262,9636617 \mu \mathrm{L} \cdot \mathrm{cm}^{-2} \cdot \mathrm{h}^{-1} ; b=0,468547664 \mathrm{cal} \cdot \mathrm{cm}^{-2} \cdot \mathrm{min}^{-1} ; c=1,230198161 ;\right.$ $\left.d=33,54420813^{\circ} \mathrm{C} ; f=0,538660895\right)$.

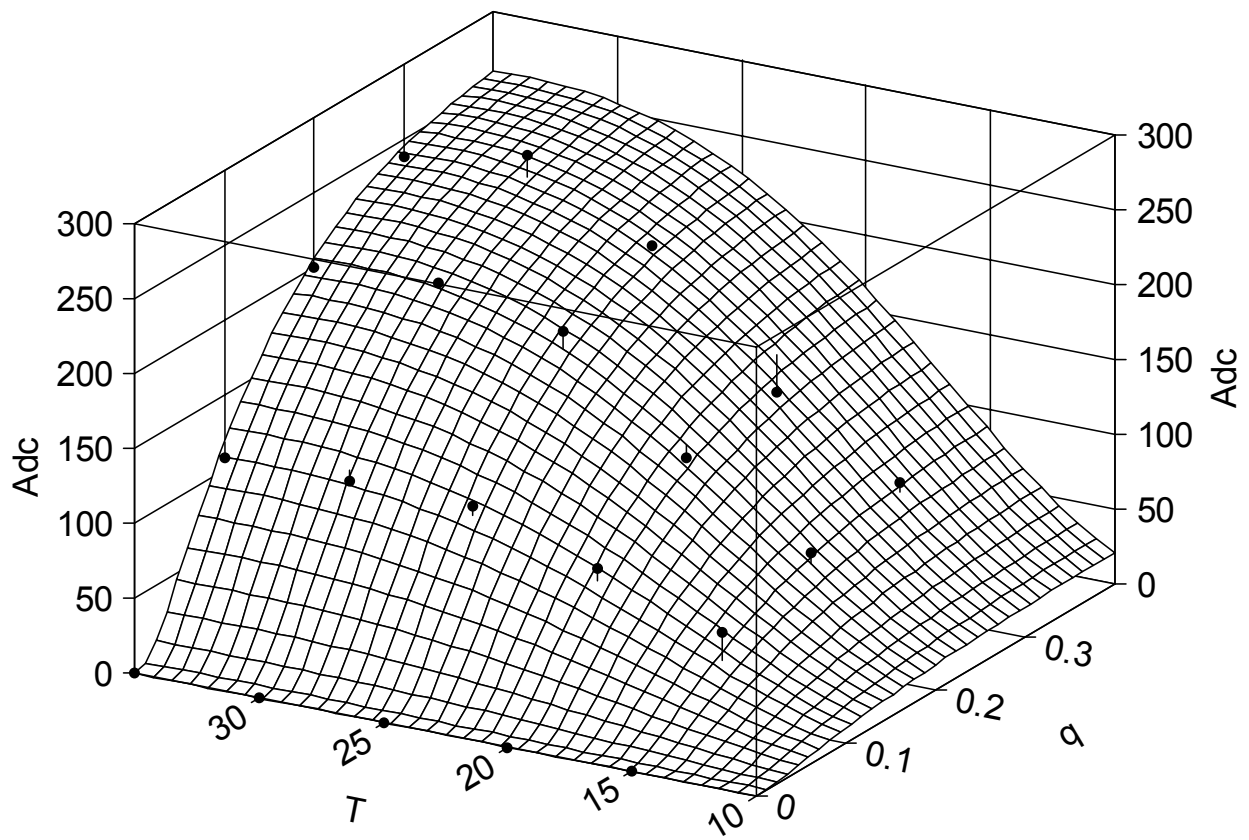

Figura 1 - Curvas de assimilação de dióxido de carbono (Adc, $\left.\mu \mathrm{L} \cdot \mathrm{cm}^{-2} \cdot \mathrm{h}^{-1}\right)$ para plantas $\mathrm{C} 4$ em função da radiação solar absorvida $\left(\mathrm{q}, \mathrm{cal} \cdot \mathrm{cm}^{-2} \cdot \mathrm{min}^{-1}\right)$ e da temperatura $\left(\mathrm{T},{ }^{\circ} \mathrm{C}\right)$ do ar (adaptado de Heemst, 1986)

Sendo as massas moleculares de $\mathrm{CO}_{2}=44 \mathrm{~g} \mathrm{~mol}^{-1}$ e de $\mathrm{CH}_{2} \mathrm{O}=30 \mathrm{~g} \mathrm{~mol}^{-1}$, a assimilação de dióxido de carbono $\left(\mu \mathrm{L} . \mathrm{cm}^{-2} \cdot \mathrm{h}^{-1}\right)$ pode ser convertida em massa bruta de carboidrato produzido ( $\mathrm{MP}_{\mathrm{CH} 2 \mathrm{O}}, \mathrm{g} \cdot \mathrm{h}^{-1} \cdot \mathrm{cm}^{-2}$ de folha), a partir da equação geral dos gases e de dados climáticos (temperatura e radiação solar absorvida).

Considerando-se a massa bruta de carboidrato produzido como sendo o valor médio diário para a duração do ciclo inteiro ( $\mathrm{DC}_{p m f}$, dias), estimado a partir do número de graus-dia, da emergência ao ponto de maturidade fisioológica $\left(G D_{p m f},{ }^{\circ} \mathrm{C} . d i a\right)$, conhecendo-se o fotoperíodo médio do ciclo $\left(H\right.$, horas.dia $\left.{ }^{-1}\right)$ e o índice de área foliar médio no ciclo $\left(I A F_{m}, \mathrm{~m}^{2} \cdot \mathrm{m}^{-2}\right)$, estimado em função do desenvolvimento relativo da cultura $\left(D r_{j}\right)$, pode-se estimar o rendimento de carboidrato total $\left(M_{C H 2 O}, \mathrm{~kg} \cdot \mathrm{ha}^{-1} \cdot \mathrm{ciclo}^{-1}\right)$, através das seguintes equações: 


$$
\begin{gathered}
M_{C_{2} O}=\frac{36,585 \cdot A d c \cdot I A F_{m} \cdot D C_{p m f} \cdot H}{T+273} \\
D C_{p m f}=\frac{G D_{p m f}}{\left(T-T_{b}\right)} \\
\alpha=\frac{\pi}{180} \operatorname{sen}\left[\frac{\pi}{180} \operatorname{sen}(d j+283) \frac{360}{365} \cdot\right] \cdot 23,5 \\
H=\frac{24}{\pi} \arccos [-\operatorname{tg}(\alpha) \cdot t g(\phi) \cdot] \\
I A F_{j}=e^{a+b \cdot D r_{j}+c \cdot \sqrt{D r_{j}}} \\
I A F_{m}=\left(\frac{\sum_{j=1}^{D C_{p m f}} I A F_{j}}{D C_{p m f}}\right) \cdot F C_{I A F} \\
D r_{j}=\frac{G D_{j}}{G D_{p m f}}=\frac{\sum_{j j=1}^{j}\left(T_{j j}-T b\right)}{\sum_{j j=1}^{j}\left(T_{j j}-T b\right)}
\end{gathered}
$$

em que $T_{b}$ se refere à temperatura basal da cultura $\left(10^{\circ} \mathrm{C}\right.$, Villa Nova et al., 1972), $T_{j j}$ à temperatuta $\left({ }^{\circ} \mathrm{C}\right)$ média diária, $\alpha$ à declinação solar (radianos) no dia juliano $(d j)$ mediano do ciclo, $\phi$ à latitude (radianos) do local, $G D_{p m f}$ ao número de graus-dia, da emergência ao ponto de maturidade fisiológica, e $a, b, c$ aos parâmetros empíricos determinados em análise de regressão $\left(\mathrm{a}=-7,63393 \mathrm{~m}^{2} \cdot \mathrm{m}^{-2} ; \mathrm{b}=-15,55692 \mathrm{~m}^{2} \cdot \mathrm{m}^{-2}\right.$; $\mathrm{c}=24,176556 \mathrm{~m}^{2} \cdot \mathrm{m}^{-2}$ ), a partir de dados obtidos por Lima (1995) para genótipos de milho com exigências calóricas alta, média e baixa. Considerando que ocorre uma variação linear do IAF para populações $\left(P_{o}\right)$ entre 4 e 6,5 plantas.m ${ }^{-2}$ (Basanta, 1999), utiliza-se um fator de correção $\left(F C_{I A F}\right)$ para ajustar o $I A F_{m}$, expresso por:

$$
F C_{I A F}=0,11764 . P_{o}+0,2915
$$

Para se transformar a massa bruta de carboidrato total final $\left(\mathrm{M}_{\mathrm{CH} 2 \mathrm{O}}\right)$ em massa de matéria seca dos diferentes órgãos (grãos, folhas, raízes, p.e.), é necessário que se façam algumas correções. 
Com base no conceito de De Wit (1965, 1982), concebido para estimar produtividade potencial de uma cultura através da energia disponível no local considerado, e a partir de dados experimentais obtidos na literatura, chegou-se às correções necessárias para estimar a produtividade potencial da cultura de milho.

\subsubsection{Correção para respiração de manutenção e crescimento (CR $\left(\mathbf{R}_{\mathrm{MC}}\right)$}

A fitomassa seca consumida nos processos de manutenção e crescimento ao longo do desenvolvimento depende principalmente da temperatura média do ar (T), sendo a correção utilizada para estimar o saldo (fotossíntese líquida) expressa por:

$$
C R_{M C}=a+b \cdot T+c T^{2}
$$

em que $a, b, c$, se referem aos parâmetros empíricos determinados em análise de regressão com base em dados obtidos de Doorenbos \& Kassam (1994), sendo $a=-0,545 ; b=0,0786^{\circ} \mathrm{C}^{-1} ; c=-0,0013^{\circ} \mathrm{C}^{-2}\left(15 \leq T \leq 35^{\circ} \mathrm{C}\right)$.

\subsubsection{Correção para interceptação de radiação solar (CRs)}

Pelo princípio da Lei de Beer-Bouguer-Lambert (Ometto, 1981), é possível estimar a interceptação da radiação solar por uma comunidade de plantas, sendo a correção da radiação solar média absorvida obtida em função do índice de área foliar médio $\left(I A F_{m}\right)$ da cultura durante o ciclo.

$$
\begin{gathered}
C R_{s}=1-e^{-k \cdot I A F_{m}} \\
\mathrm{k}=\mathrm{a}+\mathrm{b} \cdot \sqrt{\mathrm{IAF}}
\end{gathered}
$$

em que $\mathrm{k}$ se refere ao coeficiente de extinção da radiação, e $a, b$, aos parâmetros empíricos determinados em análise de regressão com base em dados obtidos de Doorenbos \& Kassam (1994), sendo $a=0,27697 \mathrm{~m}^{2} \cdot \mathrm{m}^{-2}$ e $b=-0,06097 \mathrm{~m} \cdot \mathrm{m}^{-1}$.

\subsection{4 Índice de colheita (IC)}

Refere-se à fração de fitomassa seca do órgão de interesse (normalmente grãos) colhido em relação à Fitomassa seca total $\left(\mathrm{FS}_{\mathrm{t}}\right)$ elaborada, a qual é obtida em função de dados experimentais. De acordo com dados relatados na literatura (Doorenbos \& Kassam, 1994; Lima, 1995; Gadioli, 1999; Barros, 1998; Sá, 2001), o índice de colheita 
para milho (grãos) varia de 0,3 a 0,5 , sendo o valor de 0,4 considerado satisfatório para obtenção de alta produtividade.

\subsubsection{Produtividade potencial de grãos $\left(P P_{g r}\right)$}

A produtividade potencial de grãos foi estimada considerando-se uma população de 6,5 plantas.m ${ }^{-2}\left(\mathrm{Po}_{\max }\right)$ e índice de colheita de $0,4\left(\mathrm{IC}_{\max }\right)$.

$$
P P_{\text {grãos }}=\frac{M_{\mathrm{CH}_{2} \mathrm{O}} \cdot C R_{m c} \cdot C R_{s} \cdot I C_{\max }}{1-u}
$$

em que $u$ se refere à umidade do grão após secagem, que normalmente situa-se em 13\% $\left(0,13 \mathrm{~g} \cdot \mathrm{g}^{-1}\right)$.

\subsection{Balanço hídrico}

Para elaboração do balanço hídrico, adotou-se o modelo proposto por Dourado Neto et al. (1991), que permite uma variação do coeficiente de evapotranspiração da cultura $(K c)$ e de profundidade efetiva do sistema radicular $(Z e)$ para qualquer distribuição de dados climatológicos disponíveis, utilizando o método de Thornthwaite \& Mather (1955), com algumas modificações. A escolha desse método foi baseada em resultados obtidos por Camargo (1962) e Camargo \& Sentelhas (1995), que demostraram sua viabilidade de uso para as condições do estado de São Paulo.

\subsubsection{Evapotranspiração de referência (ou Potencial)}

Evapotranspiração de referência $\left(E T_{0 i}, \mathrm{~mm}_{\text {.período }}{ }^{-1}\right)$ é definida como sendo "a perda total de água, na forma de vapor normal de uma superfície natural bem suprida de água à atmosfera, ocorrendo quando a folhagem vegetal se mantém turgescente e quando o processo decorre dependente de atributos do clima, não havendo restrição de água ou cobertura vegetal”. Calcula-se $E T_{0 i}$ utilizando-se a seguinte equação (Thornthwaite, 1948):

$$
E T_{0 i}=\gamma \cdot\left(\sigma \cdot \frac{T_{i}}{I}\right)^{a} \cdot\left(\frac{H_{i}}{12}\right) \cdot N_{i}
$$

em que $T_{i}$ refere-se à temperatura média $\left({ }^{\circ} \mathrm{C}\right), \gamma$ e $\sigma$ aos parâmetros empíricos $(\gamma=0,53$

mm.dia ${ }^{-1}$ e $\left.\sigma=10^{\circ} \mathrm{C}^{-1}\right), I$ ao índice térmico, $a$ ao coeficiente empírico composto, $N_{i}$ ao 
número de dias e $H_{i}$ ao número possível de horas de brilho solar no dia mediano do iésimo período.

\subsection{2 Índice térmico e coeficiente empírico composto}

O índice térmico $(I)$ é calculado pela seguinte equação:

$$
I=\beta_{1} \cdot \sum_{i=1}^{12} T_{i}^{\beta_{2}}
$$

em que $T_{i}$ se refere à temperatura $\left({ }^{\circ} \mathrm{C}\right)$ média diária do j-ésimo mês e $\beta_{1}$ e $\beta_{2}$ ao parâmetro empírico $\left(\beta_{1}=0,08745^{\circ} \mathrm{C}^{-\beta 2}\right.$ e $\left.\beta_{2}=1,514\right)$.

O coeficiente empírico composto $(a)$ é calculado da seguinte maneira:

$$
a=a_{0}+a_{1} \cdot I+a_{2} \cdot I^{2}+a_{3} \cdot I^{3}
$$

em que $a_{0}=0,49239 ; a_{1}=0,01792 ; a_{2}=-0,0000771 ; a_{3}=0,000000675$.

\subsubsection{Evapotranspiração máxima}

A evapotranspiração máxima $\left(E T m_{i}\right)$ representa a maior troca de gases possíveis entre a cultura e a atmosfera, e corresponde à máxima produtividade da cultura. Estimase a evapotranspiração máxima da cultura multiplicando-se a evapotranspiração de referência $\left(E T o_{i}\right)$ pelo coeficiente de evapotranspiração da cultura $\left(K c_{i}\right)$ no i-ésimo período, ou seja:

$$
E T_{m_{i}}=E T_{0_{i}} \cdot K_{c_{i}}
$$

em que $K c_{i}$ é obtido na literatura (Doorenbos \& Pruitt, 1997) através de valores tabelados em função do estádio fenológico da cultura. No presente trabalho considerouse valor um médio $(K c=1)$ para o coeficiente de cultivo durante o ciclo da cultura.

\subsubsection{Capacidade de água disponível}

Capacidade de água disponível (CAD, mm) é o armazenamento máximo de água disponível para a cultura, podendo ser calculada utilizando a seguinte expressão:

$$
C A D_{i}=\left(\theta_{c c}-\theta_{p m p}\right) \cdot Z e_{i} \cdot 10
$$

em que $Z e_{i}$ é a profundidade efetiva do sistema radicular $(\mathrm{cm})$ no i-ésimo período, $\theta_{c c}$ é a capacidade de campo $\left(\mathrm{cm}^{3} \cdot \mathrm{cm}^{-3}\right)$ e $\theta_{\text {pmp }}$ é o ponto de murcha permanente $\left(\mathrm{cm}^{3} \cdot \mathrm{cm}^{-3}\right)$. 
Nas estimativas realizadas no presente trabalho foi utilizado um valor médio padrão $(C A D=50 \mathrm{~mm})$ para o cálculo do balanço hídrico.

\subsubsection{Saldo (S) e negativo acumulado (L)}

O saldo do armazenamento é obtido pela diferença entre a precipitação e a evapotranspiração máxima da cultura no i-ésimo período.

$$
S_{i}=p p t_{i}-E T_{m i}
$$

O negativo acumulado pode ser entendido como a diferença acumulada das chuvas e da evapotranspiração potencial até o período em questão, o qual é estimado utilizando o seguinte procedimento:

Se $S_{i}<0$ :

$$
\begin{gathered}
L_{i}=L_{i-1}+\left(p p t_{i}-E T_{0_{i}}\right) \\
\operatorname{Arm}_{i}=C A D_{i} \cdot e^{\left(\frac{L_{i}}{C A D_{i}}\right)}
\end{gathered}
$$

Se $S_{i} \geq 0$ :

$$
\begin{gathered}
L_{i}=-C A D \cdot \ln \left(\frac{\text { Arm }_{i}}{C A D_{i}}\right) \\
\operatorname{Arm}_{i}=\operatorname{Arm}_{i-1}+S_{i}
\end{gathered}
$$

em que $L_{i}$ se refere ao negativo acumulado (mm), $A r m_{i}$ ao armazenamento $(\mathrm{mm})$ e $C A D_{i}$ à capacidade de água disponível $(\mathrm{mm})$ no i-ésimo período.

\subsubsection{Variação do armazenamento}

A variação do armazenamento é obtida pela diferença entre o armazenamento do período em questão $\left(\operatorname{Arm}_{i}\right)$ e o armazenamento do período anterior $\left(\operatorname{Arm}_{i-1}\right)$ :

$$
\Delta \mathrm{Arm}_{i}=\mathrm{Arm}_{i}-\mathrm{Arm}_{i-1}
$$

\subsubsection{Critérios de iniciar o balanço hídrico}

Para iniciar o balanço hídrico, Thornthwaite \& Mather (1955) consideram que o solo se encontra na capacidade de campo no final do período úmido $(L=0 ; A r m=C A D)$. Os autores assumiram que a evapotranspiração varia linearmente 
com o armazenamento de água no solo, e que o armazenamento varia exponencialmente com o negativo acumulado.

\subsubsection{Evapotranspiração real}

A evapotranspiração real é definida como a perda de água pelas plantas que realmente ocorre em função da disponibilidade de água no solo. Há duas situações distintas para o seu cálculo:

$$
\begin{gathered}
\operatorname{ETr}_{i}=\operatorname{ETm}_{i}\left(\operatorname{se} S_{i} \geq 0\right) \\
\operatorname{ETr}_{i}=\operatorname{ppt}_{i}+\Delta \operatorname{Arm}_{i}\left(\operatorname{se} S_{i}<0\right)
\end{gathered}
$$

em que $\mathrm{ppt}_{\mathrm{i}}$ se refere à chuva.

\subsubsection{Deficiência hídrica}

Deficiência hídrica $\left(D h_{i}\right)$ é definida como a diferença entre a $E T r_{i}$ e a $E T m_{i}$. Existe então deficiência hídrica nos períodos em que a $\operatorname{ETr}_{\mathrm{i}}$ é menor que a $\mathrm{ETm}_{\mathrm{i}}$, em conseqüência da soma da chuva e da variação de água armazenada no perfil do solo ocorridos, não suprir a demanda evapotranspiratória.

$$
\begin{gathered}
D h_{i}=0\left(\text { se } S_{i} \geq 0\right) \\
D h_{i}=E \operatorname{ETm}_{i}-E \operatorname{Err}_{i}\left(\operatorname{se} S_{i}<0\right)
\end{gathered}
$$

\subsubsection{Fator de depleção de produtividade $(F d)$}

A depleção de produtividade causada pela deficiência hídrica no solo é calculada pela relação entre o somatório da evapotranspiração real $\left(E_{T r}\right)$ e o somatório da evapotranspiração máxima $\left(E T m_{j}\right)$ da cultura no j-ésimo período, ou seja:

$$
F d=\frac{\sum_{j=1}^{D C_{p m f}} E T r_{j}}{\sum_{j=1}^{D C_{p m f}} E T m_{j}}
$$

\subsection{Produtividade deplecionada de grãos $\left(\boldsymbol{P}_{\text {grãos }}\right)$}

A estimação da produtividade deplecionada (em função da evapotranspiração relativa) de grãos de milho no presente trabalho foi realizada considerando o índice de 
colheita e a população de plantas como dependentes do fator de depleção de produtividade, ou seja:

$$
\begin{gathered}
P o=P o_{\text {min }}+\frac{P o_{\text {max }}-P o_{\text {min }}}{1-P o_{\text {miun }}} .\left(F d-F d_{\text {min }}\right) \\
I C=I C_{\text {min }}+\frac{I C_{\text {max }}-I C_{\text {min }}}{1-I C_{\text {min }}} .\left(F d-F d_{\text {min }}\right)
\end{gathered}
$$

em que $P o_{\min }$ se refere à população mínima $\left(4\right.$ plantas.m $\left.{ }^{-2}\right), P o_{\max }$ à população máxima $\left(6,5\right.$ plantas.m $\left.{ }^{-2}\right), I C_{\min }$ ao índice de colheita mínimo $(0,3), I C_{\max }$ ao índice de colheita máximo $(0,4)$, considerados no presente modelo, e $F d_{\min }$ ao fator de depleção mínimo encontrado durante o cálculo do fator de depleção. Dessa maneira, a redução da população de plantas causada pela deficiência hídrica no solo, afeta diretamente o índice de área foliar, utilizado no cálculo da massa bruta de carboidrato $\left(\mathrm{M}_{\mathrm{CH} 2 \mathrm{O}}\right)$.

A produtividade deplecionada de grãos de milho foi estimada utilizando-se a seguinte expressão:

$$
P_{\text {grãos }}=\frac{M_{\mathrm{CH}_{2} \mathrm{O}} \cdot C R_{m c} \cdot C R_{s} \cdot I C}{1-u}
$$

\subsection{Avaliação do modelo}

O modelo proposto foi avaliado a partir de estimativas de produtividade potencial de grãos de milho comparadas aos dados obtidos em experimentos realizados por Lima (1995), Gadioli (1999), Forsthofer et al. (2002) e Pioneer (2002).

Em cada trabalho foram avaliados genótipos de milho com diferentes exigências calóricas, semeados em épocas distintas. Os dados foram coletados em ensaios experimentais realizados nos municípios de Piracicaba, SP (Lima, 1995), Taubaté, SP (Gadioli, 1999), Porto Alegre, RS (Forsthofer et al., 2002) e Tupaciguara, MG e Barreiras, BA (Pioneer, 2002).

\subsection{Análise estatística}

Os modelos obtidos a partir de análises de regressão para estimativa da assimilação de dióxido de carbono (Equação 2), do índice de área foliar (Equação 7), do 
coeficiente de respiração de manutenção e crescimento (Equação 11) e do coeficiente de extinção da radiação solar (Equação 13) foram analisados por meio da significância (intervalo de confiança) e da análise de variância (Teste F) de seus parâmetros.

A análise estatística para avaliação do modelo de estimação da produtividade de grãos de milho consistiu na comparação dos valores de produtividade potencial obtidos nas simulações com os observados, utilizando modelo linear.

\subsection{Semivariogramas}

Para análise dos dados climáticos utilizados na elaboração dos mapas de produtividade potencial e produtividade deplecionada, utilizou-se a teoria das variáveis regionalizadas (Matheron, 1971), cuja ferramenta base é o semivariograma, definido como:

$$
\gamma(h)=\frac{1}{2 N(h)} \sum_{i=1}^{N(h)}\left[v\left(x_{i}\right)-v\left(x_{i}+h\right)\right]^{2}
$$

sendo $v\left(x_{i}\right)$ o valor da propriedade medida no local $x_{i}$ e $N(h)$ o número de pares de dados separados pelo vetor $h$.

O programa computacional TNTmips foi utilizado para construir os semivariogramas experimentais de cada variável climática e para escolha do modelo matemático teórico que melhor se ajustasse aos dados dos semivariogramas experimentais (Tabelas 3, 4 e 5).

Tabela 3. Parâmetros referentes aos modelos esféricos ajustados aos semivariogramas dos dados de temperatura utilizados na interpolação por Krigagem

\begin{tabular}{cccc}
\hline Mês & Patamar & Mês & Patamar \\
\hline janeiro & 2,160948 & julho & 2,528692 \\
fevereiro & 2,310111 & agosto & 3,139174 \\
março & 2,441744 & setembro & 3,274524 \\
abril & 2,455585 & outubro & 2,846164 \\
maio & 2,431168 & novembro & 2,427983 \\
junho & 2,647437 & dezembro & 2,534323 \\
\hline
\end{tabular}

$\mathrm{n}^{\mathrm{o}}$ de intervalos: 54; comprimento do intervalo: 0,183541; alcance: 2,936653 
Tabela 4. Parâmetros referentes aos modelos esféricos ajustados aos semivariogramas dos dados de chuva utilizados na interpolação por Krigagem

\begin{tabular}{cccccc}
\hline Mês & Alcance & Patamar & Mês & Alcance & Patamar \\
\hline Janeiro & 2,753112 & 1582,578258 & julho & 3,120193 & 452,613548 \\
fevereiro & 3,487275 & 1239,258784 & agosto & 3,303734 & 464,119093 \\
Março & 3,303734 & 1847,217986 & setembro & 3,303734 & 655,847120 \\
Abril & 3,487275 & 1376,878847 & outubro & 3,487275 & 832,152494 \\
Maio & 3,303734 & 712,778211 & novembro & 2,753112 & 1141,795107 \\
Junho & 2,753112 & 478,536553 & dezembro & 2,753112 & 1815,866568 \\
\hline
\end{tabular}

$\mathrm{n}^{\circ}$ de intervalos: 54; comprimento do intervalo: 0,183541

Tabela 5. Parâmetros referentes aos modelos esféricos ajustados aos semivariogramas dos dados de radiação solar global utilizados na interpolação por Krigagem

\begin{tabular}{cccccc}
\hline Mês & Alcance & Patamar & Mês & Alcance & Patamar \\
\hline Janeiro & 3,797104 & 314,254220 & julho & 3,586154 & 1462,894785 \\
fevereiro & 3,586154 & 563,590034 & agosto & 3,797104 & 746,317904 \\
Março & 2,953303 & 502,015293 & setembro & 3,586154 & 1770,234911 \\
Abril & 3,375204 & 972,287086 & outubro & 3,586154 & 1811,885674 \\
Maio & 3,586154 & 435,491407 & novembro & 4,008054 & 761,459393 \\
Junho & 3,586154 & 952,596545 & dezembro & 4,429955 & 544,785177 \\
\hline
\end{tabular}

$\mathrm{n}^{\mathrm{o}}$ de intervalos: 45; comprimento do intervalo: 0,210950

\subsection{Elaboração dos mapas}

Os dados de temperatura e chuva referentes a 272 municípios (265 municípios paulistas e 7 municípios de estados vizinhos, Figura 2) foram obtidos no Departamento de Ciências Exatas - ESALQ/USP ${ }^{1}$, sendo originados pelo Departamento de Águas e energia elétrica de São Paulo - DAEE e Instituto Agronômico de Campinas - IAC. Foram utilizados valores médios mensais obtidos a partir de séries de dados variando de 15 a 80 anos de coleta, dependendo da localidade. Para as demais localidades do estado

\footnotetext{
${ }^{1}$ http://ce.esalq.usp.br/bhbrasil
} 
de São Paulo, os valores médios utilizados foram estimados por meio de interpolação por Krigagem, a partir dos dados disponíveis.

Os dados de radiação solar global (média mensal) foram obtidos do Laboratório de Energia Solar/INMET, gerados pelo modelo físico BRAZILSR, com base em dados do satélite geoestacionário GOES-8. Os dados foram gerados em malhas de $0,5^{\circ}$ de latitude x $0,5^{\circ}$ de longitude, conforme apresentado na Figura 3.

Os mapas de superfície dos valores de temperatura, precipitação pluvial e radiação solar global foram elaborados por meio de interpolação por Krigagem ordinária, em malhas com espaçamento de $2 \times 2 \mathrm{~km}$, utilizando-se o mapa de contorno do estado de São Paulo como referência.

A partir dos valores médios mensais de temperatura, radiação solar global e precipitação pluvial obtidos por meio de Krigagem, para os 645 municípios do estado de São Paulo, processaram-se as simulações no software elaborado em Visual Basic para estimativa de produtividade potencial e produtividade deplecionada de grãos de milho e deficiência hídrica no solo. Esses atributos, por sua vez, foram exportados para o programa TNTmips para confecção dos mapas de superfície dos valores de produtividade potencial e produtividade deplecionada de grãos de milho e deficiência hídrica no solo para o Estado de São Paulo. 


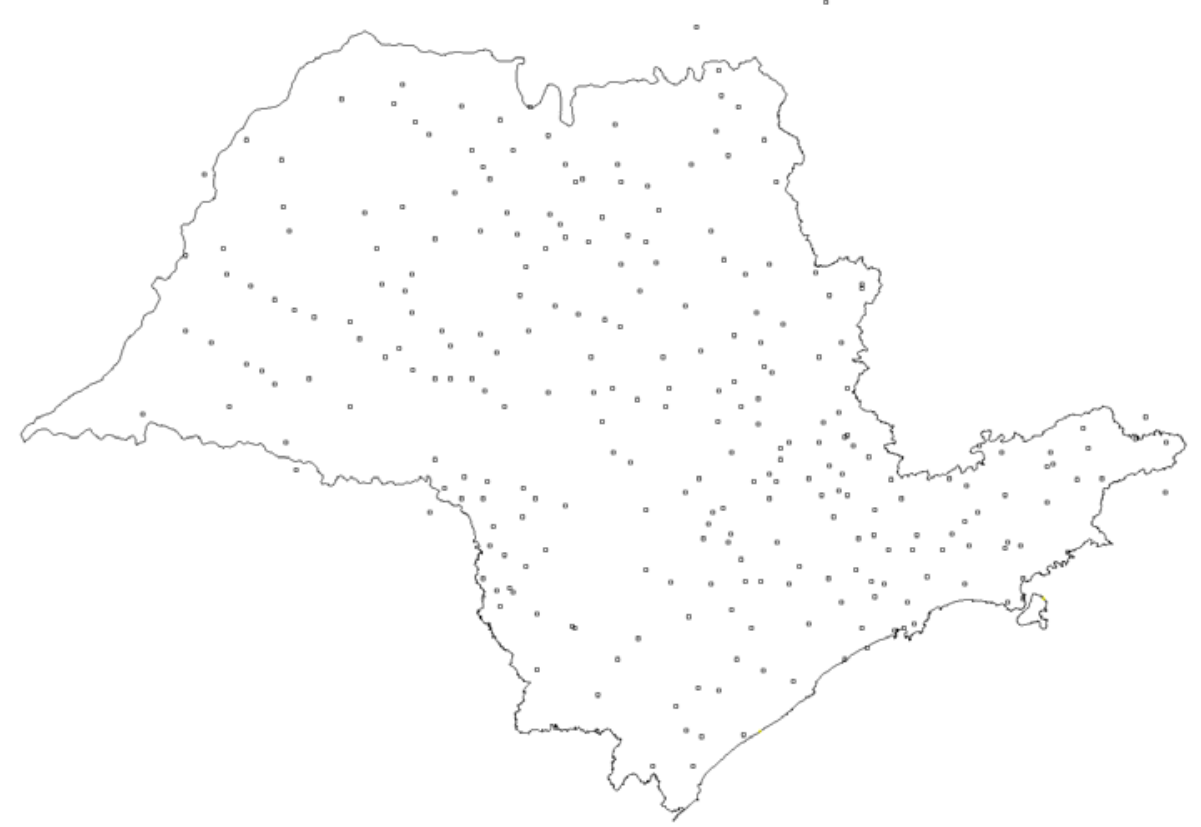

Figura 2 - Representação das estações climáticas com dados de temperatura e chuva utilizados na Krigagem

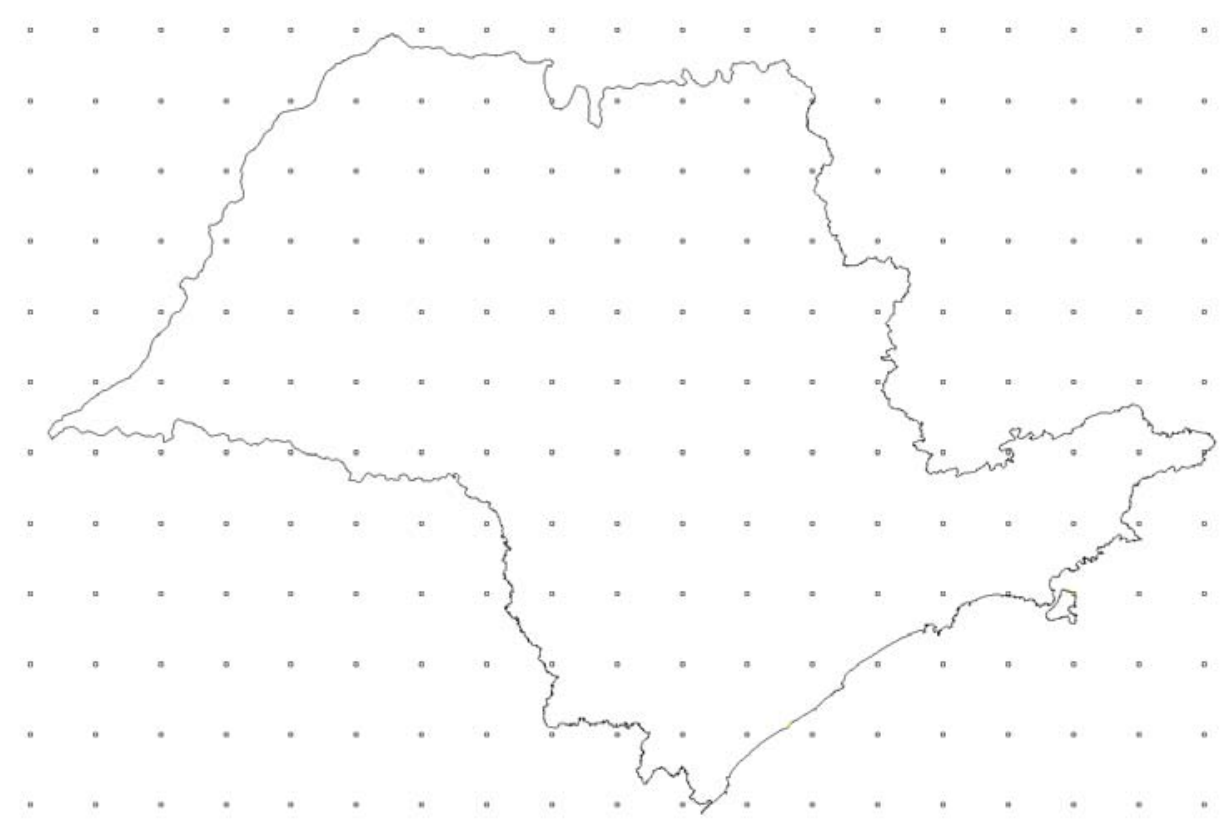

Figura 3 - Representação dos pontos com dados de radiação solar global utilizados na Krigagem 


\section{RESULTADOS E DISCUSSÃO}

\subsection{Análises de regressão}

Com base em dados obtidos na literatura, foram elaborados modelos para estimativa da assimilação de dióxido de carbono (Adc, Equação 2), do índice de área foliar (IAF, Equação 7), do coeficiente de respiração de manutenção e crescimento ( $\mathrm{CR}_{\mathrm{mc}}$, Equação 11) e do coeficiente de extinção da radiação solar (k, Equação 13), a partir de análises de regressão, sendo analisados por meio da significância (intervalo de confiança) e da análise de variância (Teste F) de seus parâmetros (Tabela 6 e Tabela 7).

Tabela 6. Coeficientes e respectivos intervalos de confiança (95\%) das análises de regressão para estimativa de $\mathrm{Adc}, \mathrm{IAF}, \mathrm{CR}_{\mathrm{mc}}$ e $\mathrm{k}$

\begin{tabular}{cccc}
\hline Coeficiente & \multicolumn{2}{c}{ Valor } & \multicolumn{2}{c}{ Intervalo de confiança } \\
& \multicolumn{2}{c}{ Assimilação de dióxido de carbono (Adc) } \\
\hline a & 262,963616 & 238,457017 & 287,470216 \\
b & 0,468548 & 0,260334 & 0,676761 \\
c & 1,230198 & 0,88544 & 1,574956 \\
d & 33,544208 & 29,87975 & 37,208666 \\
e & 0,538661 & 0,444727 & 0,632595 \\
& Índice de área foliar (IAF) \\
a & $-7,633931$ & $-10,213314$ & $-5,054548$ \\
b & $-15,55692$ & $-19,790184$ & $-11,323656$ \\
c & 24,176556 & 17,524381 & 30,828732 \\
& Coeficiente de respiração de manutenção e crescimento $\left(\mathrm{CR}_{\mathrm{mc}}\right)$ \\
$\mathrm{a}$ & $-0,545$ & $-0,72312$ & $-0,36688$ \\
$\mathrm{~b}$ & 0,0786 & 0,061242 & 0,095972 \\
$\mathrm{c}$ & $-0,0013$ & $-0,001702$ & $-0,00094$ \\
& Coeficiente de extinção da radiação solar (k) & \\
$\mathrm{a}$ & 0,27697 & 0,222437 & 0,331503 \\
$\mathrm{~b}$ & $-0,060968$ & $-0,092452$ & $-0,029483$ \\
\hline
\end{tabular}


Tabela 7. Análises de variância das análises de regressão para estimativa de Adc, IAF,

\begin{tabular}{|c|c|c|c|c|}
\hline $\mathrm{CV}$ & GL & QM & $\mathrm{F}_{\mathrm{c}}$ & $\mathrm{F}_{\mathrm{tab}}(0,01)$ \\
\hline \multicolumn{5}{|c|}{ Assimilação de dióxido de carbono (Adc) } \\
\hline Modelo & 4 & 47837,434 & 282,99 & 4,43 \\
\hline Erro & 20 & 169,045 & & \\
\hline Total & 24 & & & \\
\hline \multicolumn{5}{|c|}{ Índice de área foliar (IAF) } \\
\hline Modelo & 2 & 23,39197 & 245,46 & 9,55 \\
\hline Erro & 7 & 0,095299 & & \\
\hline Total & 9 & & & \\
\hline \multicolumn{5}{|c|}{ Coeficiente de respiração de manutenção e crescimento $\left(\mathrm{CR}_{\mathrm{mc}}\right)$} \\
\hline Modelo & 2 & 0,100533 & 296,3 & 30,81 \\
\hline Erro & 3 & 0,000339 & & \\
\hline Total & 5 & & & \\
\hline \multicolumn{5}{|c|}{ Coeficiente de extinção da radiação solar (k) } \\
\hline Modelo & 1 & 0,003521 & 37,42 & 34,12 \\
\hline Erro & 3 & 0,0000941 & & \\
\hline Total & 4 & & & \\
\hline
\end{tabular}

$\mathrm{F}_{\mathrm{c}}=$ valor de $\mathrm{F}$ calculado na análise de variância; $\mathrm{F}_{\mathrm{tab}}(0,01)=$ valor de $\mathrm{F}$ tabelado ao nível de significância de $1 \%$.

Os modelos selecionados atenderam aos critérios estabelecidos para escolha dos mesmos, ou seja, todos os parâmetros foram significativos, e o valor de $\mathrm{F}$ calculado foi sempre maior que o $\mathrm{F}$ tabelado com nível de significância de 1\%. Desta maneira, as Equações 2, 7, 11 e 13 podem ser utilizadas para estimativas de produtividade da cultura de milho com o procedimento apresentado.

\subsection{Variáveis climáticas}

Nas Figuras 4 e 5, pode-se observar os valores médios de temperatura e radiação solar global, respectivamente, no Estado de São Paulo, para os períodos que apresentaram os valores extremos (inferior e superior) destas variáveis. 

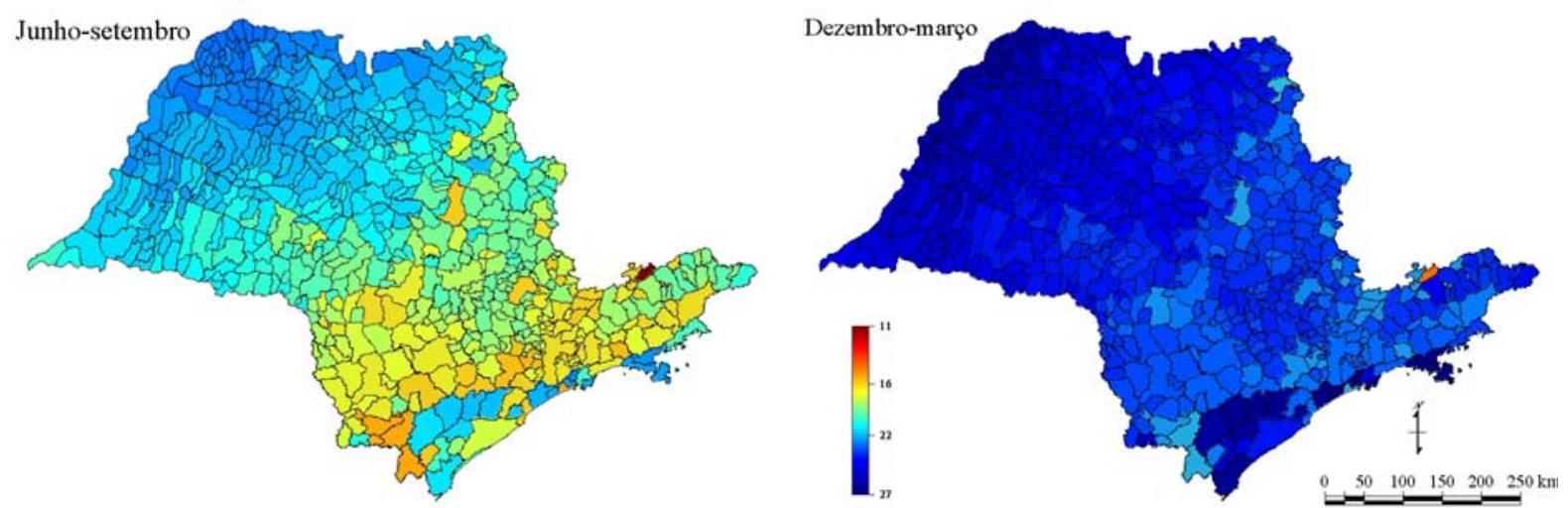

Figura 4 - Temperatura $\left({ }^{\circ} \mathrm{C}\right)$ média do ar nos meses de junho a setembro e dezembro a março no estado de São Paulo
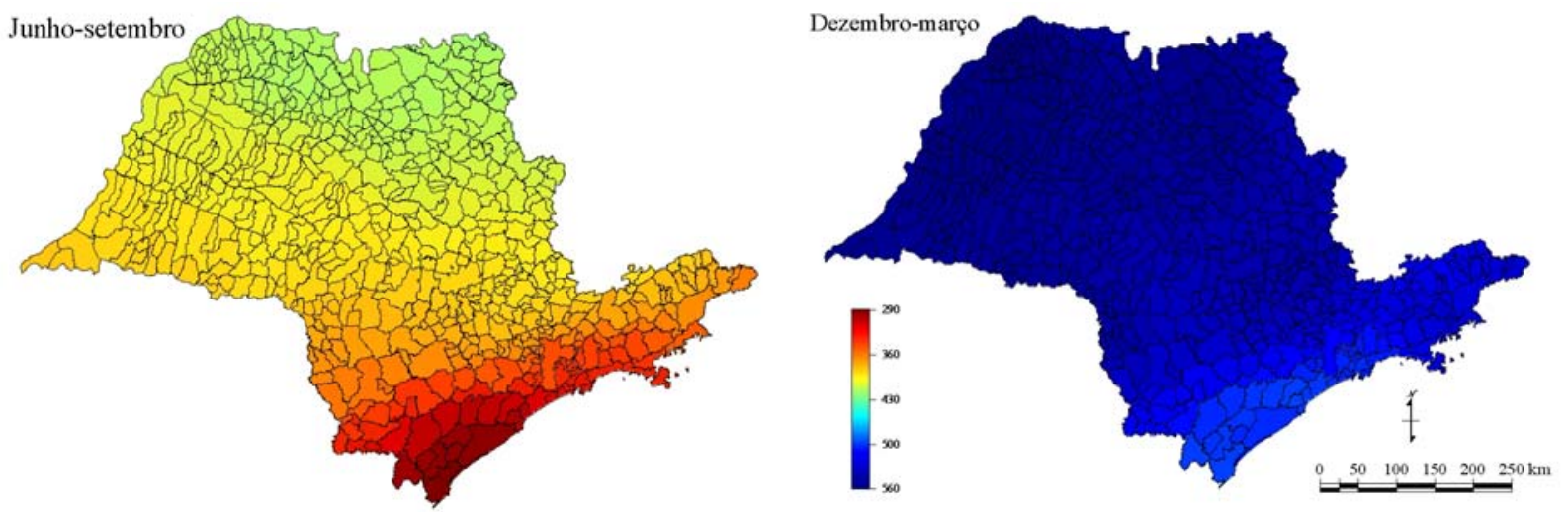

Figura 5 - Radiação solar global $\left(\right.$ cal. $\left.\mathrm{cm}^{-2} \cdot \mathrm{dia}^{-1}\right)$ nos meses de junho a setembro e dezembro a março no Estado de São Paulo

\subsection{Estimativas de produtividade potencial de grãos de milho}

O modelo proposto foi avaliado a partir de estimativas de produtividade potencial de grãos de milho comparadas aos dados obtidos em experimentos realizados por Lima (1995), Gadioli (1999), Forsthofer (2002) e Pioneer (2002), sendo o resumo da avaliação apresentado na Figura 6 e na Tabela 8. A comparação realizada é possível, porque tanto os valores estimados, quanto os valores observados, referem-se à produtividade potencial de grãos de milho em condições adequadas de suprimento de água e nutrientes, sem sofrer injúrias por pragas e doenças, e sem competição com plantas daninhas. 


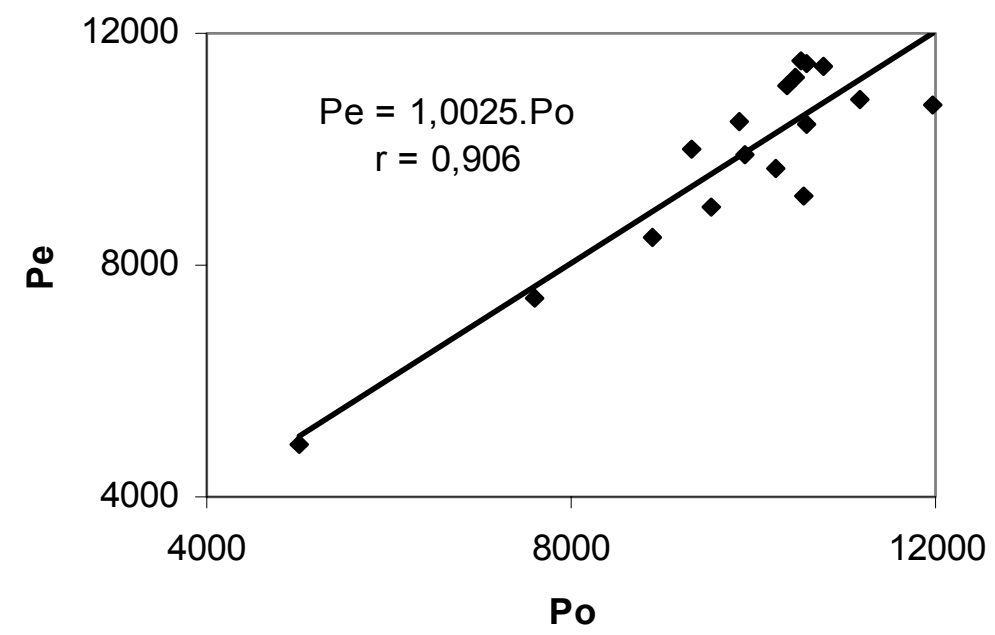

Figura 6 - Comparação entre produtividades potenciais de grãos de milho observadas $\left(\mathrm{P}_{\mathrm{o}}, \mathrm{kg}_{\mathrm{h}} \mathrm{h}^{-1}\right)$ (Lima, 1995; Gadioli, 1999; Pioneer, 2002; Forsthofer et al., 2002) e estimada $\left(\mathrm{P}_{\mathrm{e}}, \mathrm{kg} \cdot \mathrm{ha}^{-1}\right)$ pelo modelo proposto

Tabela 8. Produtividades potenciais de grãos de milho observadas $\left(\mathrm{P}_{\mathrm{o}}\right)$ e estimadas $\left(\mathrm{P}_{\mathrm{e}}\right) \mathrm{e}$ os respectivos desvios $(\mathrm{d}, \%)$

\begin{tabular}{ccccc}
\hline Fonte & Local & $\mathrm{P}_{\mathrm{o}}\left(\mathrm{kg} \cdot \mathrm{ha}^{-1}\right)$ & $\mathrm{P}_{\mathrm{e}}\left(\mathrm{kg} \cdot \mathrm{ha}^{-1}\right)$ & $\mathrm{d}^{1}$ \\
\hline Lima (1995) & Piracicaba-SP & 11.157 & 10.873 & $-2,55$ \\
Lima (1995) & Piracicaba-SP & 10.765 & 11.413 & 6,02 \\
Lima (1995) & Piracicaba-SP & 10.577 & 11.458 & 8,33 \\
Lima (1995) & Piracicaba-SP & 11.961 & 10.762 & $-10,02$ \\
Lima (1995) & Piracicaba-SP & 10.470 & 11.222 & 7,18 \\
Lima (1995) & Piracicaba-SP & 10.508 & 11.511 & 9,55 \\
Gadioli (1999) & Taubaté-SP & 10.553 & 9.181 & $-13,00$ \\
Gadioli (1999) & Taubaté-SP & 9.859 & 10.470 & 6,20 \\
Gadioli (1999) & Taubaté-SP & 10.258 & 9.662 & $-5,81$ \\
Gadioli (1999) & Taubaté-SP & 10.570 & 10.413 & $-1,49$ \\
Gadioli (1999) & Taubaté-SP & 9.321 & 10.019 & 7,49 \\
Gadioli (1999) & Taubaté-SP & 9.551 & 8.985 & $-5,93$ \\
Forsthofer et al. (2002) & Porto Alegre-RS & 7.610 & 7.446 & $-2,16$ \\
Forsthofer et al. (2002) & Porto Alegre-RS & 10.380 & 11.100 & 6,94 \\
Forsthofer et al. (2002) & Porto Alegre-RS & 5.023 & 4.900 & $-2,45$ \\
Pioneer (2002) & Tapacigura-MG & 8.900 & 8.500 & $-4,49$ \\
Pioneer (2002) & Barreiras-BA & 9.900 & 9.908 & 0,08 \\
\hline
\end{tabular}

${ }^{1} d(\%)=\frac{P e-P o}{P o} \times 100$ 
De acordo as simulações realizadas, pode-se observar que os erros de estimativa ultrapassaram 10\% apenas em duas situações (Tabela 8). Os resultados indicam que as produtividades potenciais estimadas pelo modelo proposto apresentaram ajuste satisfatório aos dados utilizados (Figura 6). Para calibração do modelo, foram utilizadas produtividades potenciais (observadas) de grãos de milho variando de 5.023 a 11.961 kg.ha ${ }^{-1}$, em cinco diferentes localidades, onde os desvios variaram de $-13 \%$ (subestimativa) em Taubaté, SP a 9,55\% (superestimativa) em Piracicaba, SP (Tabela 8).

Os mapas com atribuição de valores para as estimativas de produtividade potencial de grãos de milho, com épocas de início do ciclo (emergência das plântulas) de janeiro a dezembro no Estado de São Paulo, são apresentados nas Figuras 7 e 8.

Vale ressaltar que o início do ciclo corresponde à fase de emergência das plântulas, sendo a duração do ciclo o período entre a emergência e o ponto de maturidade fisiológica.

De acordo com os resultados das estimativas de produtividade potencial de milho no estado de São Paulo, pode-se perceber que, para todo o estado, as maiores produtividades foram observadas com início do ciclo a partir do mês de outubro até janeiro. Isto porque o ciclo da cultura coincide com as épocas em que a temperatura do ar e a radiação solar estão mais elevadas. Por outro lado, a diminuição dos valores de temperatura do ar e de radiação solar a partir de fevereiro, causa uma redução na produtividade potencial (Figuras 7 e 8 ).

Cruz et al. (1994) comentam que o cultivo de milho, com semeadura no período de fevereiro a abril (safrinha), é comum entre os produtores que procuram um melhor preço para o produto, porém com obtenção de produtividades mais baixas.

De acordo com estes resultados, portanto, pode-se inferir que o modelo proposto apresenta coerência nas estimativas realizadas, tendo em vista que as épocas de início do ciclo mais recomendadas, são aquelas em que ocorrem as maiores produtividades potenciais de grãos de milho no Estado de São Paulo (Conab, 2004b), ou seja, na safra normal, quando a disponibilidade de energia e água no sistema é mais elevada.

As áreas que apresentaram os maiores valores de produtividade potencial de grãos de milho são aquelas situadas na região Centro-Oeste do estado, para todas as 
épocas simuladas, indicando que estas localidades apresentam maior potencial para o cultivo de milho.
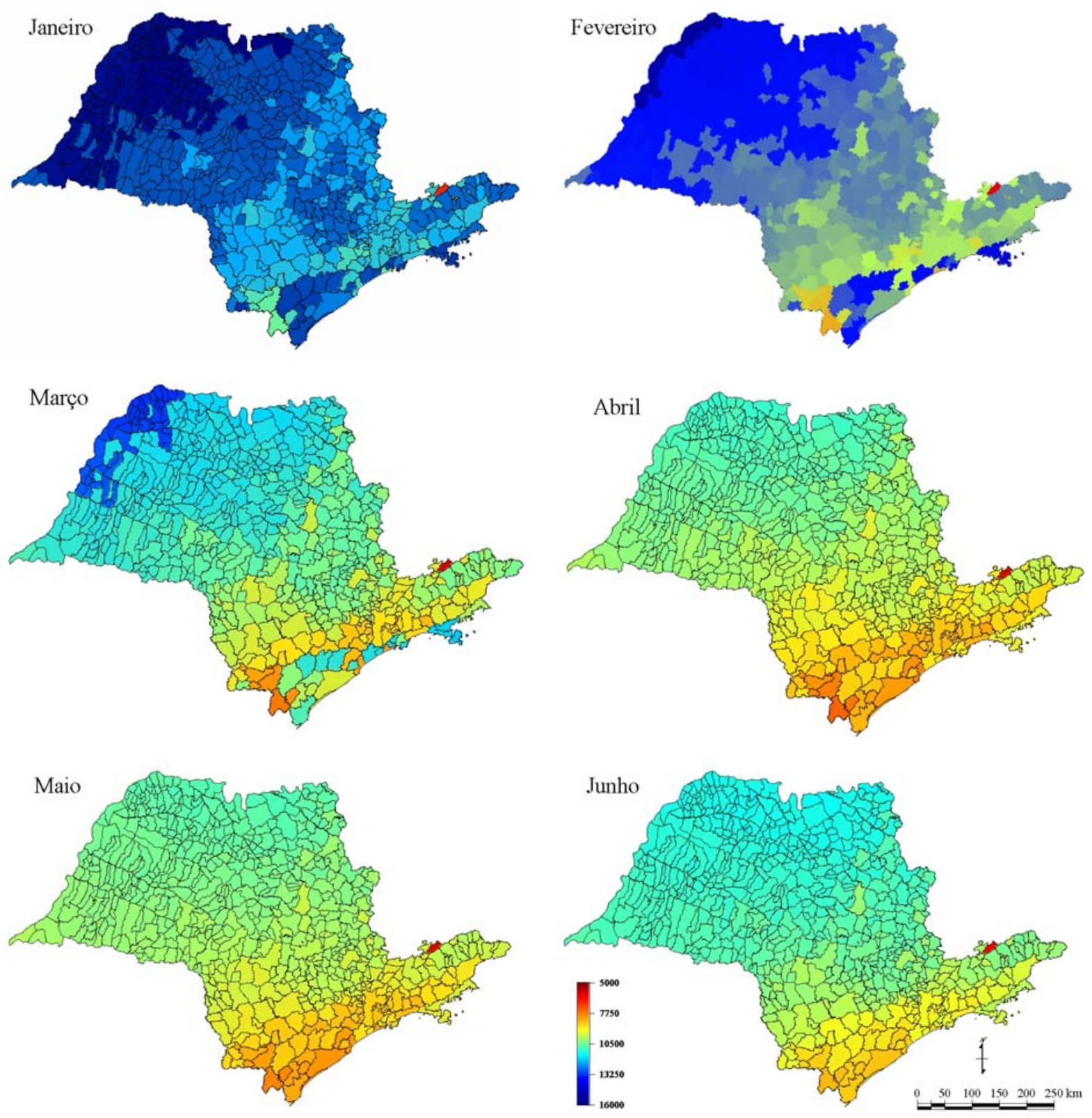

Figura 7 - Produtividade potencial $\left(\mathrm{kg} \cdot \mathrm{ha}^{-1}\right)$ de grãos de milho no estado de São Paulo estimada com início do ciclo no primeiro dia dos meses de janeiro, fevereiro, março, abril, maio e junho 

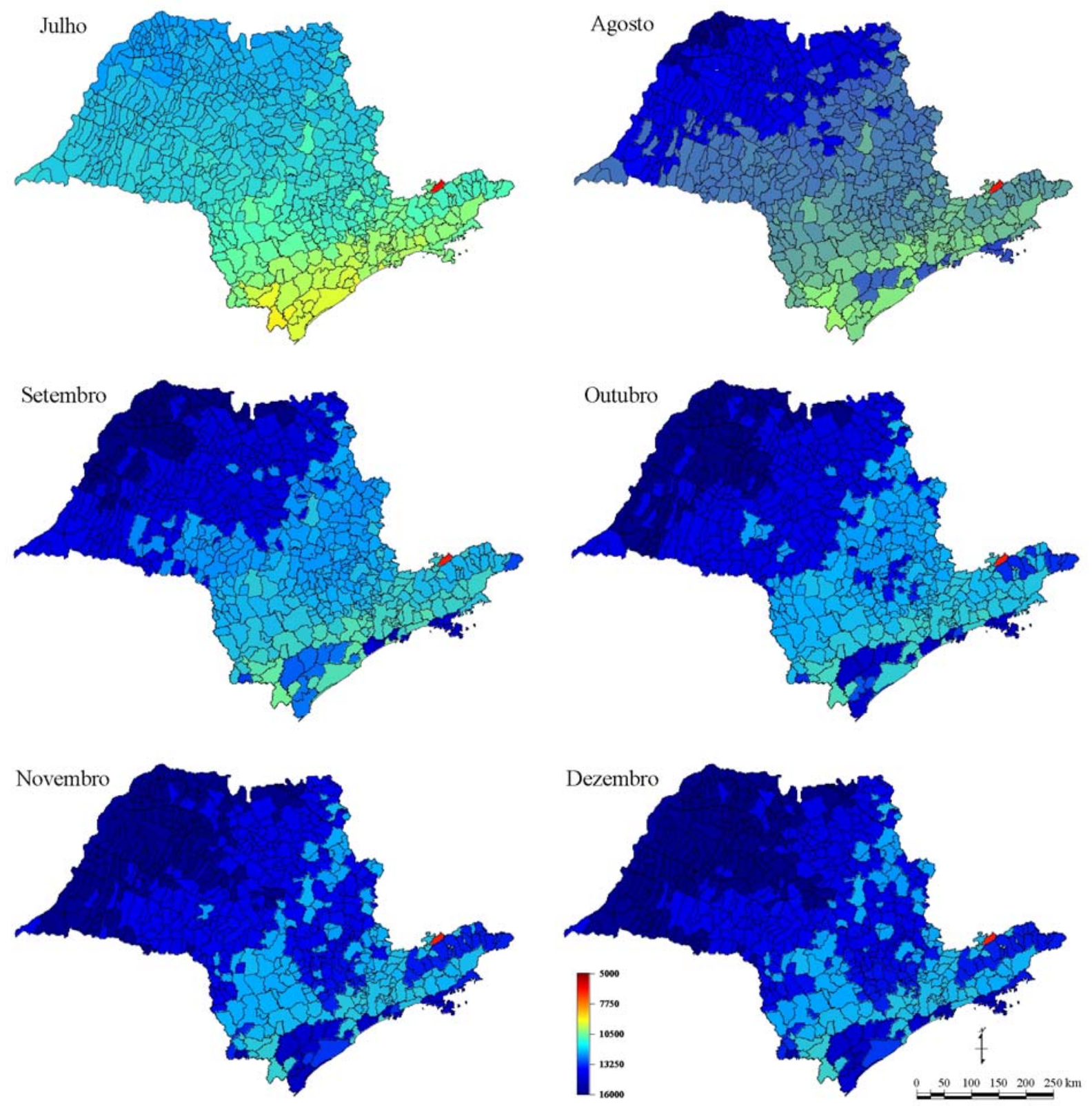

Figura 8 - Produtividade potencial $\left(\mathrm{kg}_{\mathrm{h}} \mathrm{ha}^{-1}\right)$ de grãos de milho no estado de São Paulo estimada com início do ciclo no primeiro dia dos meses de julho, agosto, setembro, outubro, novembro e dezembro

O potencial de produtividade de grãos de milho, conforme estudos teóricos, com simulações feitas com o uso de computadores, para as condições do cinturão do milho

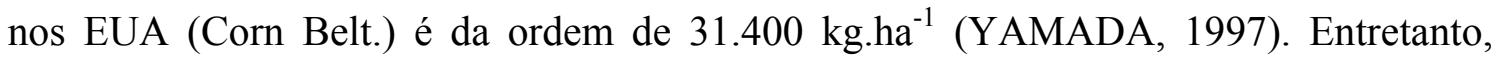


poucos dados são disponíveis relatando produtividades recordes de milho no campo. De acordo com Vyn (2001), há relatos do agricultor Herman Warsaw do Estado de Illinois, EUA, que em 1985 obteve $23.200 \mathrm{~kg} \cdot \mathrm{ha}^{-1}$, e do agricultor Francis Child do Estado de Iowa, EUA, que em 1999 obteve o recorde de $24.700 \mathrm{~kg} \cdot \mathrm{ha}^{-1}$.

No Brasil, a partir da década de 70, foram instituídos os concursos de produtividade de milho, coordenados pelas instituições oficiais de assistência técnica, pesquisa e firmas produtoras de sementes. Além do caráter educacional e da transferência de tecnologias aos agricultores, busca-se também, com base nas tecnologias disponíveis, a obtenção de altas produtividades de milho (Coelho et al., 2003). Na Tabela 9 são apresentados os resultados dos campeões de produtividade de milho no Brasil.

Tabela 9. Campeões nacionais de produtividade de milho no Brasil no período de 1981 a 1999

\begin{tabular}{cllc}
\hline $\begin{array}{c}\text { Ano } \\
\text { agrícola }\end{array}$ & \multicolumn{1}{c}{ Agricultor } & \multicolumn{1}{c}{ Local } & Produtividade $\left({\left.\mathrm{kg} . h \mathrm{ha}^{-1}\right)}^{\mathrm{c}}\right.$ \\
\hline $1981 / 1982$ & Walter Bernades & Alegre, ES & 14.677 \\
$1982 / 1983$ & Ailton Novais & Pratápolis, MG & 13.436 \\
$1983 / 1984$ & José A.B. Cardoso & Batatais, SP & 15.138 \\
$1984 / 1985$ & José G. Cerqueira & Codisburgo, MG & 14.110 \\
$1985 / 1986$ & Marcelo C. Madeira & Divinolândia, MG & 15.563 \\
$1986 / 1987$ & Bauke D. Dijkstra & Ponta Grossa, PR & 15.777 \\
$1987 / 1988$ & Lister F. Fernandes & Ituverava, SP & 16.058 \\
$1988 / 1989$ & Sebastião A. Silva & Coromandel, MG & 14.666 \\
$1989 / 1990$ & Nercy S. Santos & Bonito, MS & 15.665 \\
$1990 / 1991$ & Sebastião G. Souza & Bonito, MS & 15.738 \\
$1991 / 1992$ & Romildo F. Dias & Capinópolis, MG & 15.740 \\
$1992 / 1993$ & Antonio P. Marques & Sabinópolis, MG & 15.990 \\
$1993 / 1994$ & Geraldo N. Lacerda & Virginópolis, MG & 16.828 \\
$1994 / 1995$ & David G. Nascimento & P. do Rio Grande, MG & 15.389 \\
$1995 / 1996$ & Ademar B. Melo & Carmo do Cajuru, MG & 15.786 \\
$1996 / 1997$ & Geniplo F. Silva & Carmo do Cajuru, MG & 13.989 \\
$1997 / 1998$ & Lázaro E. Rabelo & Coromel, MG & 12.750 \\
$1998 / 1999$ & Paulo C. Cabral & Alterosa, MG & 13.369 \\
\hline
\end{tabular}

Fonte: Coelho et al. (2003)

Com base nesses resultados, observa-se que o recorde de produtividade de grãos de milho no Brasil é de $16.800 \mathrm{~kg} \cdot \mathrm{ha}^{-1}$, obtido no município de Virginópolis, MG, no 
ano de 1994. No Estado de São Paulo, a produtividade máxima alcançada foi de 16.058 kg.ha ${ }^{-1}$, na safra 1987/1988. Esses resultados, portanto, confirmam o potencial produtivo da cultura do milho estimado pelo presente modelo, demonstrando que a ordem de grandeza dos valores simulados para o Estado de São Paulo, está de acordo com as observações de campo registradas por agricultores.

Vale ressaltar que, a interpretação dos resultados apresentados nas Figuras 7 e 8, deve estar associada aos resultados apresentados na Tabela 10. Isto porque se adotou como critério de recomendação do cultivo de milho em cada época de semeadura, a ocorrência do florescimento da cultura em até 75 dias, estimado a partir das exigências calóricas dos genótipos classificados como normal $\left(950^{\circ} \mathrm{C}\right.$.dia $)$, precoce $\left(850^{\circ} \mathrm{C}\right.$.dia $)$ ou superprecoce $\left(750^{\circ} \mathrm{C}\right.$.dia). O genótipo é recomendado considerando-se 60 dias como sendo ótimo para o período entre a emergência e o florescimento, selecionando-se aquele que apresenta soma calórica mais próxima deste período. Na Tabela 10, portanto, há indicação do genótipo mais recomendado quanto ao ciclo para cada município do estado de São Paulo, nas doze épocas de semeadura simuladas.

\subsection{Deficiência hídrica}

Na Figura 9 pode-se observar os valores médios de deficiência hídrica no Estado de São Paulo, para os períodos que apresentaram os valores extremos (inferior e superior) desta variável.
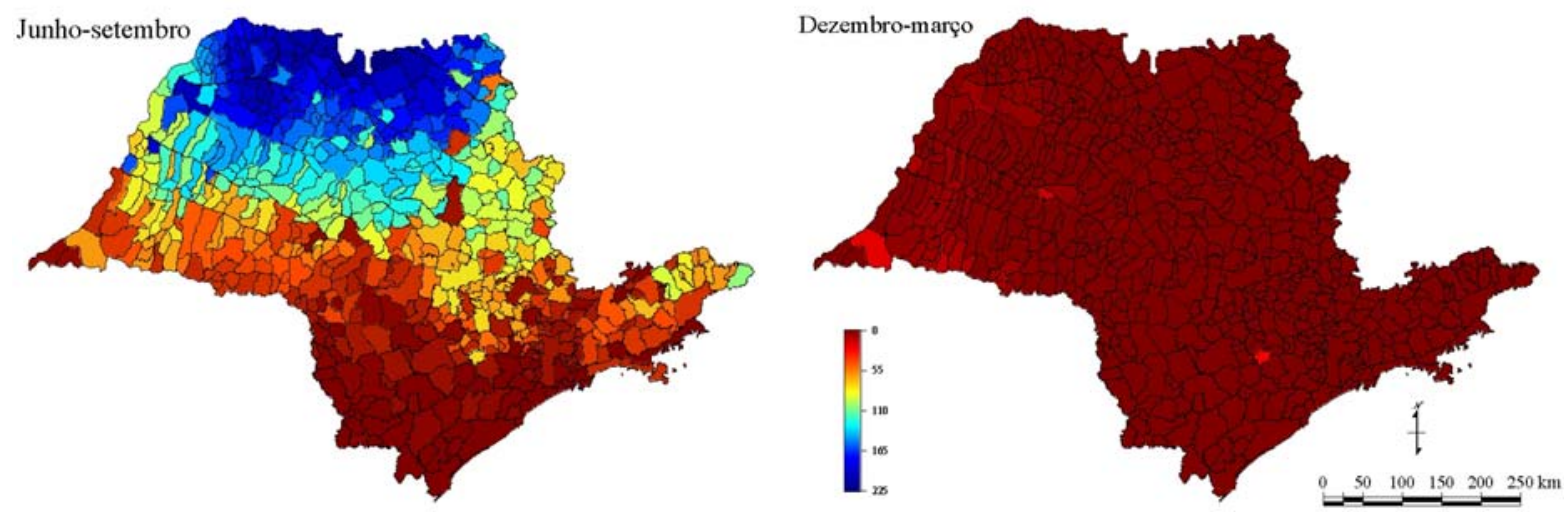

Figura 9 - Deficiência hídrica $(\mathrm{mm})$ do solo no período referente aos meses de junho a setembro, e dezembro a março no estado de São Paulo 
A deficiência hídrica no solo como fator limitante da produtividade de grãos de milho é bastante pesquisada (Castro, 1979; Matzenauer et al., 1995; Doorenbos \& Kassam, 1994; Alfonsi et al., 1997), sendo seu conhecimento imprescindível para o manejo da cultura. Os períodos de maior (dezembro a março) e menor (junho a setembro) oferta hídrica no solo são apresentados (Figura 9) como indicativo das épocas mais apropriadas para cada regime de cultivo, ou seja, sequeiro ou irrigado, respectivamente.

Durante o período compreendido entre os meses de dezembro a março, foram observados baixos valores de deficiência hídrica no solo, para todo o estado, com valores sempre inferiores a $25 \mathrm{~mm}$ (Figura 9).

As áreas que apresentaram os maiores valores de deficiência hídrica no solo, no período de junho a setembro (Figura 9), são aquelas situadas na região Noroeste do estado, indicando que estas localidades apresentam limitações para o cultivo de milho no regime de sequeiro com início do ciclo entre os meses de abril a setembro.

\subsection{Estimativas de produtividade deplecionada de grãos de milho}

As estimativas de produtividade deplecionada de grãos de milho com início de ciclo de janeiro a dezembro no Estado de São Paulo são apresentadas nas Figuras 10 e 11.

De acordo com os resultados das estimativas de produtividade deplecionada de grãos de milho, pode-se perceber que os maiores valores, para todo o estado de São Paulo, foram observados com épocas de semeadura nos meses de outubro, novembro e dezembro. Isto ocorre porque, tanto a disponibilidade de energia no sistema é elevada (Figuras 5 e 6) nesta época do ano, resultando em altas produtividades potenciais (Figuras 7 e 8), como a deficiência hídrica nos meses de dezembro a março é relativamente baixa (Figura 9) em grande parte do estado, propiciando o atendimento hídrico da cultura nas fases mais sensíveis ao estresse hídrico. Por outro lado, a elevação da deficiência hídrica observada nos meses de junho a setembro (Figura 9), causou uma redução na produtividade deplecionada para os ciclos iniciados a partir de março até setembro, especialmente nas áreas situadas na região Noroeste do estado. 

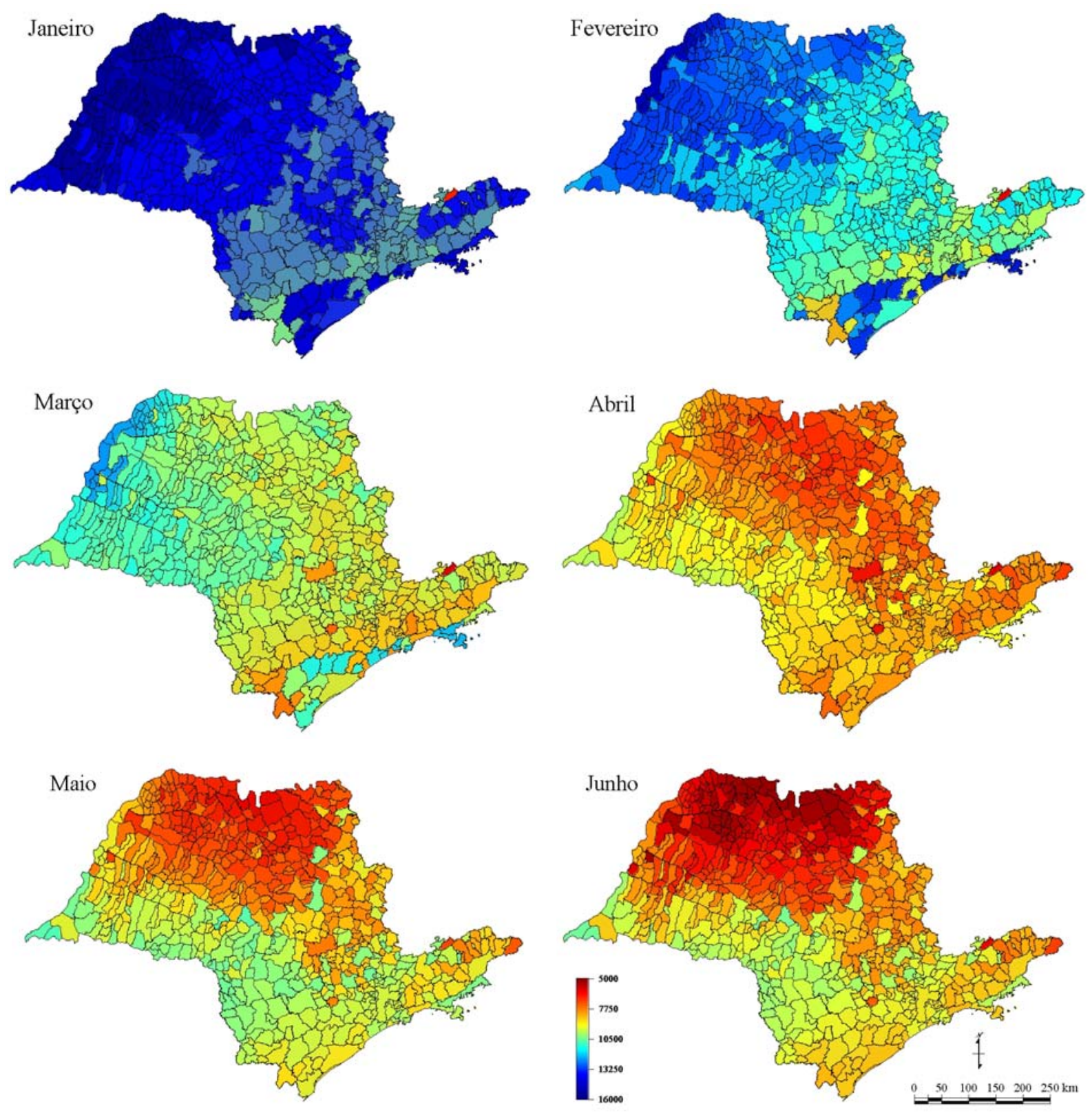

Figura 10 - Produtividade deplecionada $\left(\mathrm{kg} \cdot \mathrm{ha}^{-1}\right)$ de grãos de milho no estado de São Paulo estimada com início do ciclo no primeiro dia dos meses de janeiro, fevereiro, março, abril, maio e junho 

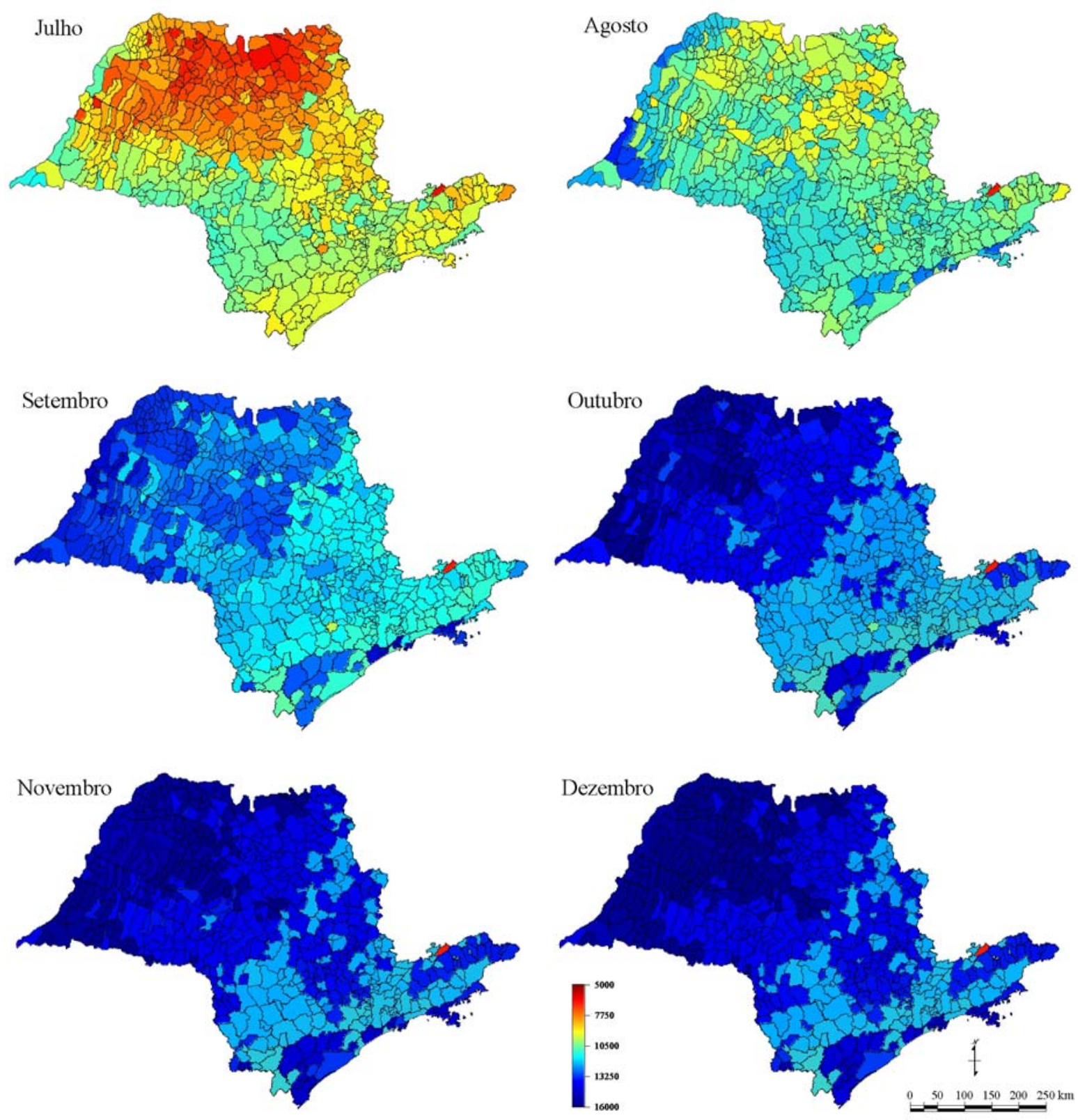

Figura 11 - Produtividade deplecionada $\left(\mathrm{kg} \cdot \mathrm{ha}^{-1}\right)$ de grãos de milho no estado de São Paulo estimada com início do ciclo no primeiro dia dos meses julho, agosto, setembro, outubro, novembro e dezembro

Estes resultados, portanto, corroboram com aqueles encontrados por Alfonsi et al. (1997), que encontraram as maiores probabilidades de atendimento hídrico para o milho semeado em outubro, novembro e dezembro em seis municípios paulistas. 
Fazendo-se uma comparação dos resultados de produtividade potencial com os de produtividade deplecionada para início de ciclo nos meses de agosto e setembro, pode-se perceber que o fator limitante do processo produtivo nesta época do ano seria a disponibilidade hídrica, já que os valores de produtividade potencial (função de temperatura e radiação solar) são relativamente elevados, especialmente na região Noroeste do estado.

Desta maneira, a exploração da cultura do milho com início do ciclo a partir do mês de agosto, pode ser viável em muitas localidades do estado de São Paulo, com a utilização de irrigação. Informações pessoais de alguns pesquisadores e produtores relatam que o cultivo de milho nesta época do ano está se tornando prática comum no estado de São Paulo. 
Tabela 10. Genótipos de milho mais recomendados para cada início de ciclo nos municípios do Estado de São Paulo, durante os doze meses do ano. N: normal; P: precoce; S: superprecoce; NR: não recomendável

\begin{tabular}{|c|c|c|c|c|c|c|c|c|c|c|c|c|}
\hline \multirow{2}{*}{ Município } & \multicolumn{12}{|c|}{ Meses } \\
\hline & Jan & $\mathrm{Fev}$ & Mar & Abr & Mai & Jun & Jul & Ago & Set & Out & Nov & Dez \\
\hline Adamantina & $\mathrm{P}$ & $\mathrm{P}$ & $\mathrm{S}$ & $\mathrm{S}$ & $\mathrm{S}$ & $\mathrm{S}$ & $\mathrm{S}$ & $\mathrm{S}$ & $\mathrm{P}$ & $\mathrm{P}$ & $\mathrm{P}$ & $\mathrm{N}$ \\
\hline Adolfo & $\mathrm{N}$ & $\mathrm{P}$ & $\mathrm{S}$ & $\mathrm{S}$ & $\mathrm{S}$ & $\mathrm{S}$ & $\mathrm{S}$ & $\mathrm{P}$ & $\mathrm{P}$ & $\mathrm{N}$ & $\mathrm{N}$ & $\mathrm{N}$ \\
\hline Aguai & $\mathrm{S}$ & $\mathrm{S}$ & $\mathrm{S}$ & NR & NR & NR & $\mathrm{S}$ & $\mathrm{S}$ & $\mathrm{S}$ & $\mathrm{S}$ & $\mathrm{P}$ & $\mathrm{P}$ \\
\hline Águas da Prata & $\mathrm{P}$ & $\mathrm{S}$ & $\mathrm{S}$ & NR & NR & $\mathrm{S}$ & $\mathrm{S}$ & $\mathrm{S}$ & $\mathrm{S}$ & $\mathrm{P}$ & $\mathrm{P}$ & $\mathrm{P}$ \\
\hline Águas de Lindóia & $\mathrm{S}$ & $\mathrm{S}$ & NR & NR & NR & NR & $\mathrm{S}$ & $\mathrm{S}$ & $\mathrm{S}$ & $\mathrm{S}$ & $\mathrm{S}$ & $\mathrm{S}$ \\
\hline Águas de Santa Barbara & $\mathrm{P}$ & $\mathrm{S}$ & $\mathrm{S}$ & NR & NR & $\mathrm{S}$ & $\mathrm{S}$ & $\mathrm{S}$ & $\mathrm{S}$ & $\mathrm{P}$ & $\mathrm{P}$ & $\mathrm{P}$ \\
\hline Águas de Sao Pedro & $\mathrm{S}$ & $\mathrm{S}$ & $\mathrm{S}$ & NR & NR & NR & $\mathrm{S}$ & $\mathrm{S}$ & $\mathrm{S}$ & $\mathrm{S}$ & $\mathrm{P}$ & $\mathrm{P}$ \\
\hline Agudos & $\mathrm{P}$ & $\mathrm{S}$ & $\mathrm{S}$ & NR & NR & NR & $\mathrm{S}$ & $\mathrm{S}$ & $\mathrm{S}$ & $\mathrm{P}$ & $\mathrm{P}$ & $\mathrm{P}$ \\
\hline Alambari & $\mathrm{S}$ & $\mathrm{S}$ & NR & NR & NR & NR & $\mathrm{S}$ & $\mathrm{S}$ & $\mathrm{S}$ & $\mathrm{S}$ & $\mathrm{S}$ & $\mathrm{S}$ \\
\hline Alfredo Marcondes & $\mathrm{N}$ & $\mathrm{P}$ & $\mathrm{S}$ & $\mathrm{S}$ & $\mathrm{S}$ & $\mathrm{S}$ & $\mathrm{S}$ & $\mathrm{P}$ & $\mathrm{P}$ & $\mathrm{N}$ & $\mathrm{N}$ & $\mathrm{N}$ \\
\hline Altair & $P$ & $\mathrm{P}$ & $\mathrm{S}$ & $\mathrm{S}$ & $\mathrm{S}$ & $\mathrm{S}$ & $\mathrm{S}$ & $\mathrm{P}$ & $\mathrm{P}$ & $\mathrm{P}$ & $\mathrm{P}$ & $\mathrm{P}$ \\
\hline Altinópolis & $\mathrm{S}$ & $\mathrm{S}$ & NR & NR & NR & NR & $\mathrm{S}$ & $\mathrm{S}$ & $\mathrm{S}$ & $\mathrm{S}$ & $\mathrm{S}$ & $\mathrm{S}$ \\
\hline Alto Alegre & $\mathrm{P}$ & $\mathrm{S}$ & $\mathrm{S}$ & $\mathrm{S}$ & $\mathrm{S}$ & $\mathrm{S}$ & $\mathrm{S}$ & $\mathrm{S}$ & $\mathrm{P}$ & $\mathrm{P}$ & $\mathrm{P}$ & $\mathrm{P}$ \\
\hline Alumínio & $\mathrm{P}$ & $\mathrm{S}$ & NR & NR & NR & NR & $\mathrm{S}$ & $\mathrm{S}$ & $\mathrm{S}$ & $\mathrm{S}$ & $\mathrm{P}$ & $\mathrm{P}$ \\
\hline Álvares Florence & $\mathrm{N}$ & $\mathrm{P}$ & $\mathrm{S}$ & $\mathrm{S}$ & $\mathrm{S}$ & $\mathrm{S}$ & $\mathrm{S}$ & $\mathrm{P}$ & $\mathrm{N}$ & $\mathrm{N}$ & $\mathrm{N}$ & $\mathrm{N}$ \\
\hline Álvares Machado & $\mathrm{N}$ & $\mathrm{P}$ & $\mathrm{S}$ & $\mathrm{S}$ & $\mathrm{S}$ & $\mathrm{S}$ & $\mathrm{S}$ & $\mathrm{P}$ & $\mathrm{P}$ & $\mathrm{N}$ & $\mathrm{N}$ & $\mathrm{N}$ \\
\hline Álvaro de Carvalho & $\mathrm{P}$ & $\mathrm{P}$ & $\mathrm{S}$ & $\mathrm{S}$ & $\mathrm{S}$ & $\mathrm{S}$ & $\mathrm{S}$ & $\mathrm{S}$ & $\mathrm{P}$ & $\mathrm{P}$ & $\mathrm{P}$ & $\mathrm{P}$ \\
\hline Alvinlândia & $\mathrm{P}$ & $\mathrm{S}$ & $\mathrm{S}$ & NR & NR & $\mathrm{S}$ & $\mathrm{S}$ & $\mathrm{S}$ & $\mathrm{S}$ & $\mathrm{P}$ & $\mathrm{P}$ & $\mathrm{P}$ \\
\hline Americana & $\mathrm{P}$ & $\mathrm{S}$ & $\mathrm{S}$ & NR & NR & $\mathrm{S}$ & $\mathrm{S}$ & $\mathrm{S}$ & $\mathrm{S}$ & $\mathrm{P}$ & $\mathrm{P}$ & $\mathrm{P}$ \\
\hline Américo Brasiliense & $\mathrm{S}$ & $\mathrm{S}$ & NR & NR & NR & NR & $\mathrm{S}$ & $\mathrm{S}$ & $\mathrm{S}$ & $\mathrm{S}$ & $\mathrm{S}$ & $\mathrm{S}$ \\
\hline Américo de Campos & $\mathrm{P}$ & $\mathrm{P}$ & $\mathrm{S}$ & $\mathrm{S}$ & $\mathrm{S}$ & $\mathrm{S}$ & $\mathrm{S}$ & $\mathrm{P}$ & $\mathrm{P}$ & $\mathrm{N}$ & $\mathrm{N}$ & $\mathrm{N}$ \\
\hline Amparo & $\mathrm{S}$ & $\mathrm{S}$ & NR & NR & NR & NR & $\mathrm{S}$ & $\mathrm{S}$ & $\mathrm{S}$ & $\mathrm{S}$ & $\mathrm{S}$ & $\mathrm{P}$ \\
\hline Analândia & $\mathrm{S}$ & $\mathrm{S}$ & NR & NR & NR & NR & $\mathrm{S}$ & $\mathrm{S}$ & $\mathrm{S}$ & $\mathrm{S}$ & $\mathrm{S}$ & $\mathrm{P}$ \\
\hline Andradina & $\mathrm{N}$ & $\mathrm{P}$ & $\mathrm{S}$ & $\mathrm{S}$ & $\mathrm{S}$ & $\mathrm{S}$ & $\mathrm{S}$ & $\mathrm{P}$ & $\mathrm{N}$ & $\mathrm{N}$ & $\mathrm{N}$ & $\mathrm{N}$ \\
\hline Angatuba & $\mathrm{S}$ & $\mathrm{S}$ & NR & NR & NR & NR & NR & $\mathrm{S}$ & $\mathrm{S}$ & $\mathrm{S}$ & $\mathrm{S}$ & $\mathrm{S}$ \\
\hline Anhembi & $\mathrm{P}$ & $\mathrm{S}$ & $\mathrm{S}$ & NR & NR & NR & $\mathrm{S}$ & $\mathrm{S}$ & $\mathrm{S}$ & $\mathrm{S}$ & $\mathrm{P}$ & $\mathrm{P}$ \\
\hline Anhumas & $\mathrm{N}$ & $\mathrm{P}$ & $\mathrm{S}$ & $\mathrm{S}$ & $\mathrm{S}$ & $\mathrm{S}$ & $\mathrm{S}$ & $\mathrm{P}$ & $\mathrm{P}$ & $\mathrm{P}$ & $\mathrm{N}$ & $\mathrm{N}$ \\
\hline Aparecida & $\mathrm{P}$ & $\mathrm{S}$ & $\mathrm{S}$ & NR & NR & NR & $\mathrm{S}$ & $\mathrm{S}$ & $\mathrm{S}$ & $\mathrm{S}$ & $\mathrm{P}$ & $\mathrm{P}$ \\
\hline Aparecida D'oeste & $\mathrm{N}$ & $\mathrm{P}$ & $\mathrm{P}$ & $\mathrm{S}$ & $\mathrm{S}$ & $\mathrm{S}$ & $\mathrm{P}$ & $\mathrm{P}$ & $\mathrm{N}$ & $\mathrm{N}$ & $\mathrm{N}$ & $\mathrm{N}$ \\
\hline Apiaí & $\mathrm{S}$ & NR & NR & NR & NR & NR & NR & NR & NR & $\mathrm{S}$ & $\mathrm{S}$ & $\mathrm{S}$ \\
\hline Aracariguama & $\mathrm{P}$ & $\mathrm{S}$ & $\mathrm{S}$ & NR & NR & NR & $\mathrm{S}$ & $\mathrm{S}$ & $\mathrm{S}$ & $\mathrm{S}$ & $\mathrm{P}$ & $\mathrm{P}$ \\
\hline Aracatuba & $\mathrm{N}$ & $\mathrm{P}$ & $\mathrm{S}$ & $\mathrm{S}$ & $\mathrm{S}$ & $\mathrm{S}$ & $\mathrm{S}$ & $\mathrm{P}$ & $\mathrm{P}$ & $\mathrm{N}$ & $\mathrm{N}$ & $\mathrm{N}$ \\
\hline Aracoiaba da Serra & $\mathrm{S}$ & $\mathrm{S}$ & NR & NR & NR & NR & NR & $\mathrm{S}$ & $\mathrm{S}$ & $\mathrm{S}$ & $\mathrm{S}$ & $\mathrm{P}$ \\
\hline Aramina & $\mathrm{P}$ & $\mathrm{S}$ & $\mathrm{S}$ & $\mathrm{S}$ & $\mathrm{S}$ & $\mathrm{S}$ & $\mathrm{S}$ & $\mathrm{P}$ & $\mathrm{P}$ & $\mathrm{P}$ & $\mathrm{P}$ & $\mathrm{P}$ \\
\hline Arandu & $\mathrm{P}$ & $\mathrm{S}$ & $\mathrm{S}$ & NR & NR & NR & $\mathrm{S}$ & $\mathrm{S}$ & $\mathrm{S}$ & $\mathrm{S}$ & $\mathrm{P}$ & $\mathrm{P}$ \\
\hline Arapei & $\mathrm{P}$ & $\mathrm{S}$ & $\mathrm{S}$ & NR & NR & NR & $\mathrm{S}$ & $\mathrm{S}$ & $\mathrm{S}$ & $\mathrm{P}$ & $\mathrm{P}$ & $\mathrm{P}$ \\
\hline Araraquara & $\mathrm{S}$ & $\mathrm{S}$ & $\mathrm{S}$ & NR & NR & $\mathrm{S}$ & $\mathrm{S}$ & $\mathrm{S}$ & $\mathrm{S}$ & $\mathrm{S}$ & $\mathrm{P}$ & $\mathrm{P}$ \\
\hline Araras & $\mathrm{P}$ & $\mathrm{S}$ & $\mathrm{S}$ & NR & NR & NR & $\mathrm{S}$ & $\mathrm{S}$ & $\mathrm{S}$ & S & $\mathrm{P}$ & $\mathrm{P}$ \\
\hline Arco-Íris & $\mathrm{P}$ & $\mathrm{P}$ & $\mathrm{S}$ & $\mathrm{S}$ & $\mathrm{S}$ & $\mathrm{S}$ & $\mathrm{S}$ & $\mathrm{P}$ & $\mathrm{P}$ & $\mathrm{P}$ & $\mathrm{N}$ & $\mathrm{N}$ \\
\hline Arealva & $\mathrm{P}$ & $\mathrm{P}$ & $\mathrm{S}$ & $\mathrm{S}$ & $\mathrm{S}$ & $\mathrm{S}$ & $\mathrm{S}$ & $\mathrm{S}$ & $\mathrm{P}$ & $\mathrm{P}$ & $\mathrm{P}$ & $\mathrm{P}$ \\
\hline Areias & $\mathrm{P}$ & $\mathrm{S}$ & $\mathrm{S}$ & NR & NR & NR & $\mathrm{S}$ & $\mathrm{S}$ & $\mathrm{S}$ & $\mathrm{P}$ & $\mathrm{P}$ & $\mathrm{P}$ \\
\hline Areiopolis & $\mathrm{P}$ & $\mathrm{S}$ & $\mathrm{S}$ & NR & NR & $\mathrm{S}$ & $\mathrm{S}$ & $\mathrm{S}$ & $\mathrm{S}$ & $\mathrm{P}$ & $\mathrm{P}$ & $\mathrm{P}$ \\
\hline Ariranha & $\mathrm{P}$ & $\mathrm{P}$ & $\mathrm{S}$ & $\mathrm{S}$ & $\mathrm{S}$ & $\mathrm{S}$ & $\mathrm{S}$ & $\mathrm{P}$ & $\mathrm{P}$ & $\mathrm{P}$ & $\mathrm{P}$ & $\mathrm{P}$ \\
\hline
\end{tabular}


Tabela 10. Genótipos de milho mais recomendados para cada início de ciclo nos municípios do Estado de São Paulo, durante os doze meses do ano. N: normal; P: precoce; S: superprecoce; NR: não recomendável

\begin{tabular}{|c|c|c|c|c|c|c|c|c|c|c|c|c|}
\hline \multirow{2}{*}{ Município } & \multicolumn{12}{|c|}{ Meses } \\
\hline & Jan & Fev & Mar & Abr & Mai & Jun & Jul & Ago & Set & Out & Nov & Dez \\
\hline Artur Nogueira & $\mathrm{S}$ & $\mathrm{S}$ & $\mathrm{S}$ & NR & NR & $\mathrm{S}$ & $\mathrm{S}$ & $\mathrm{S}$ & $\mathrm{S}$ & $\mathrm{S}$ & $\mathrm{P}$ & $\mathrm{P}$ \\
\hline Arujá & $\mathrm{S}$ & NR & NR & NR & NR & NR & NR & $\mathrm{S}$ & $\mathrm{S}$ & $\mathrm{S}$ & $\mathrm{S}$ & $\mathrm{S}$ \\
\hline Asasia & $\mathrm{N}$ & $\mathrm{N}$ & $\mathrm{P}$ & $\mathrm{S}$ & $\mathrm{S}$ & $\mathrm{S}$ & $\mathrm{P}$ & $\mathrm{N}$ & $\mathrm{N}$ & $\mathrm{N}$ & $\mathrm{N}$ & $\mathrm{N}$ \\
\hline Assis & $\mathrm{P}$ & $\mathrm{S}$ & $\mathrm{S}$ & $\mathrm{S}$ & $\mathrm{S}$ & $\mathrm{S}$ & $\mathrm{S}$ & $\mathrm{S}$ & $\mathrm{P}$ & $\mathrm{P}$ & $\mathrm{P}$ & $\mathrm{P}$ \\
\hline Atibaia & $\mathrm{S}$ & NR & NR & NR & NR & NR & NR & $\mathrm{S}$ & $\mathrm{S}$ & $\mathrm{S}$ & $\mathrm{S}$ & $\mathrm{S}$ \\
\hline Auriflama & $\mathrm{N}$ & $\mathrm{P}$ & $\mathrm{S}$ & $\mathrm{S}$ & $\mathrm{S}$ & $\mathrm{S}$ & $\mathrm{P}$ & $\mathrm{P}$ & $\mathrm{N}$ & $\mathrm{N}$ & $\mathrm{N}$ & $\mathrm{N}$ \\
\hline Avaí & $\mathrm{P}$ & $\mathrm{P}$ & $\mathrm{S}$ & $\mathrm{S}$ & $\mathrm{S}$ & $\mathrm{S}$ & $\mathrm{S}$ & $\mathrm{S}$ & $\mathrm{P}$ & $\mathrm{P}$ & $\mathrm{P}$ & $\mathrm{P}$ \\
\hline Avanhangava & $\mathrm{P}$ & $\mathrm{P}$ & $\mathrm{S}$ & $\mathrm{S}$ & $\mathrm{S}$ & $\mathrm{S}$ & $\mathrm{S}$ & $\mathrm{P}$ & $\mathrm{P}$ & $\mathrm{P}$ & $\mathrm{N}$ & $\mathrm{N}$ \\
\hline Avaré & $\mathrm{S}$ & NR & NR & NR & NR & NR & NR & NR & $\mathrm{S}$ & $\mathrm{S}$ & $\mathrm{S}$ & $\mathrm{S}$ \\
\hline Bady Bassitt & $\mathrm{P}$ & $\mathrm{P}$ & $\mathrm{S}$ & $\mathrm{S}$ & $\mathrm{S}$ & $\mathrm{S}$ & $\mathrm{S}$ & $\mathrm{P}$ & $\mathrm{P}$ & $\mathrm{P}$ & $\mathrm{N}$ & $\mathrm{N}$ \\
\hline Balbinos & $\mathrm{P}$ & $\mathrm{P}$ & $\mathrm{S}$ & $\mathrm{S}$ & $\mathrm{S}$ & $\mathrm{S}$ & $\mathrm{S}$ & $\mathrm{S}$ & $\mathrm{P}$ & $\mathrm{P}$ & $\mathrm{P}$ & $\mathrm{N}$ \\
\hline Bálsamo & $\mathrm{P}$ & $\mathrm{S}$ & $\mathrm{S}$ & $\mathrm{S}$ & $\mathrm{S}$ & $\mathrm{S}$ & $\mathrm{S}$ & $\mathrm{P}$ & $\mathrm{P}$ & $\mathrm{P}$ & $\mathrm{P}$ & $\mathrm{P}$ \\
\hline Bananal & $\mathrm{P}$ & $\mathrm{S}$ & $\mathrm{S}$ & $\mathrm{S}$ & $\mathrm{S}$ & $\mathrm{S}$ & $\mathrm{S}$ & $\mathrm{S}$ & $\mathrm{P}$ & $\mathrm{P}$ & $\mathrm{P}$ & $\mathrm{P}$ \\
\hline Barão de Antonina & $\mathrm{P}$ & $\mathrm{S}$ & NR & NR & NR & NR & $\mathrm{S}$ & $\mathrm{S}$ & $\mathrm{S}$ & $\mathrm{S}$ & $\mathrm{P}$ & $\mathrm{P}$ \\
\hline Barbosa & $\mathrm{N}$ & $\mathrm{P}$ & $\mathrm{S}$ & $\mathrm{S}$ & $\mathrm{S}$ & $\mathrm{S}$ & $\mathrm{S}$ & $\mathrm{P}$ & $\mathrm{P}$ & $\mathrm{N}$ & $\mathrm{N}$ & $\mathrm{N}$ \\
\hline Bariri & $\mathrm{P}$ & $\mathrm{P}$ & $\mathrm{S}$ & $\mathrm{S}$ & $\mathrm{S}$ & $\mathrm{S}$ & $\mathrm{S}$ & $\mathrm{S}$ & $\mathrm{P}$ & $\mathrm{P}$ & $\mathrm{P}$ & $\mathrm{N}$ \\
\hline Barra Bonita & $\mathrm{P}$ & $\mathrm{S}$ & $\mathrm{S}$ & $\mathrm{S}$ & $\mathrm{S}$ & $\mathrm{S}$ & $\mathrm{S}$ & $\mathrm{S}$ & $\mathrm{S}$ & $\mathrm{P}$ & $\mathrm{P}$ & $\mathrm{P}$ \\
\hline Barra do Chapéu & $\mathrm{S}$ & $\mathrm{S}$ & NR & NR & NR & NR & NR & $\mathrm{S}$ & $\mathrm{S}$ & $\mathrm{S}$ & $\mathrm{S}$ & $\mathrm{P}$ \\
\hline Barra do Turvo & $\mathrm{S}$ & NR & NR & NR & NR & NR & NR & NR & NR & $\mathrm{S}$ & $\mathrm{S}$ & $\mathrm{S}$ \\
\hline Barretos & $\mathrm{P}$ & $\mathrm{P}$ & $\mathrm{S}$ & $\mathrm{S}$ & $\mathrm{S}$ & $\mathrm{S}$ & $\mathrm{S}$ & $\mathrm{P}$ & $\mathrm{P}$ & $\mathrm{P}$ & $\mathrm{P}$ & $\mathrm{P}$ \\
\hline Barrinha & $\mathrm{P}$ & $\mathrm{S}$ & $\mathrm{S}$ & $\mathrm{S}$ & $\mathrm{S}$ & $\mathrm{S}$ & $\mathrm{S}$ & $\mathrm{S}$ & $\mathrm{P}$ & $\mathrm{P}$ & $\mathrm{P}$ & $\mathrm{P}$ \\
\hline Barueri & $\mathrm{S}$ & $\mathrm{S}$ & NR & NR & NR & NR & $\mathrm{S}$ & $\mathrm{S}$ & $\mathrm{S}$ & $\mathrm{S}$ & $\mathrm{S}$ & $\mathrm{S}$ \\
\hline Bastos & $P$ & $P$ & $\mathrm{~S}$ & $\mathrm{~S}$ & $\mathrm{~S}$ & $\mathrm{~S}$ & $\mathrm{~S}$ & $\mathrm{P}$ & $\mathrm{P}$ & $\mathrm{P}$ & $\mathrm{N}$ & $\mathrm{N}$ \\
\hline Batatais & $\mathrm{P}$ & $\mathrm{S}$ & $\mathrm{S}$ & $\mathrm{S}$ & $\mathrm{S}$ & $\mathrm{S}$ & $\mathrm{S}$ & $\mathrm{S}$ & $\mathrm{P}$ & $\mathrm{P}$ & $\mathrm{P}$ & $\mathrm{P}$ \\
\hline Bauru & $\mathrm{P}$ & $\mathrm{S}$ & $\mathrm{S}$ & NR & NR & $\mathrm{S}$ & $\mathrm{S}$ & $\mathrm{S}$ & $\mathrm{S}$ & $\mathrm{P}$ & $\mathrm{P}$ & $\mathrm{P}$ \\
\hline Bebedouro & $\mathrm{P}$ & $\mathrm{S}$ & $\mathrm{S}$ & $\mathrm{S}$ & $\mathrm{S}$ & $\mathrm{S}$ & $\mathrm{S}$ & $\mathrm{P}$ & $\mathrm{P}$ & $\mathrm{P}$ & $\mathrm{P}$ & $\mathrm{P}$ \\
\hline Bento de Abreu & $\mathrm{N}$ & $\mathrm{P}$ & $\mathrm{S}$ & $\mathrm{S}$ & $\mathrm{S}$ & $\mathrm{S}$ & $\mathrm{S}$ & $\mathrm{P}$ & $\mathrm{N}$ & $\mathrm{N}$ & $\mathrm{N}$ & $\mathrm{N}$ \\
\hline Bernardino de Campo & $\mathrm{S}$ & $\mathrm{S}$ & NR & NR & NR & NR & NR & $\mathrm{S}$ & $\mathrm{S}$ & $\mathrm{S}$ & $\mathrm{S}$ & $\mathrm{S}$ \\
\hline Bertioga & $\mathrm{S}$ & $\mathrm{S}$ & NR & NR & NR & NR & NR & $\mathrm{S}$ & $\mathrm{S}$ & $\mathrm{S}$ & $\mathrm{S}$ & $\mathrm{S}$ \\
\hline Bilac & $\mathrm{P}$ & $\mathrm{P}$ & $\mathrm{S}$ & $\mathrm{S}$ & $\mathrm{S}$ & $\mathrm{S}$ & $\mathrm{S}$ & $\mathrm{P}$ & $\mathrm{P}$ & $\mathrm{P}$ & $\mathrm{N}$ & $\mathrm{N}$ \\
\hline Birigui & $\mathrm{N}$ & $\mathrm{P}$ & $\mathrm{S}$ & $\mathrm{S}$ & $\mathrm{S}$ & $\mathrm{S}$ & $\mathrm{S}$ & $\mathrm{P}$ & $\mathrm{P}$ & $\mathrm{N}$ & $\mathrm{N}$ & $\mathrm{N}$ \\
\hline Biritiba-Mirim & $\mathrm{S}$ & $\mathrm{S}$ & NR & NR & NR & NR & NR & $\mathrm{S}$ & $\mathrm{S}$ & $\mathrm{S}$ & $\mathrm{S}$ & $\mathrm{S}$ \\
\hline Boa Esperança do Sul & $\mathrm{P}$ & $\mathrm{P}$ & $\mathrm{S}$ & $\mathrm{S}$ & $\mathrm{S}$ & $\mathrm{S}$ & $\mathrm{S}$ & $\mathrm{P}$ & $\mathrm{P}$ & $\mathrm{P}$ & $\mathrm{N}$ & $\mathrm{N}$ \\
\hline Bocaina & $\mathrm{P}$ & $\mathrm{P}$ & $\mathrm{S}$ & $\mathrm{S}$ & $\mathrm{S}$ & $\mathrm{S}$ & $\mathrm{S}$ & $\mathrm{P}$ & $\mathrm{P}$ & $\mathrm{P}$ & $\mathrm{N}$ & $\mathrm{N}$ \\
\hline Bofete & $\mathrm{S}$ & $\mathrm{S}$ & NR & NR & NR & NR & $\mathrm{S}$ & $\mathrm{S}$ & $\mathrm{S}$ & $\mathrm{S}$ & $\mathrm{S}$ & $\mathrm{S}$ \\
\hline Boituva & $\mathrm{S}$ & $\mathrm{S}$ & NR & NR & NR & NR & NR & $\mathrm{S}$ & $\mathrm{S}$ & $\mathrm{S}$ & $\mathrm{S}$ & $\mathrm{P}$ \\
\hline Bom Jesus dos Perdões & $\mathrm{S}$ & $\mathrm{S}$ & NR & NR & NR & NR & NR & $\mathrm{S}$ & $\mathrm{S}$ & $\mathrm{S}$ & $\mathrm{S}$ & $\mathrm{S}$ \\
\hline Bom Sucesso de Itararé & $\mathrm{P}$ & $\mathrm{S}$ & NR & NR & NR & NR & NR & $\mathrm{S}$ & $\mathrm{S}$ & $\mathrm{S}$ & $\mathrm{P}$ & $\mathrm{P}$ \\
\hline Bora & $\mathrm{P}$ & $\mathrm{P}$ & $\mathrm{S}$ & $\mathrm{S}$ & $\mathrm{S}$ & $\mathrm{S}$ & $\mathrm{S}$ & $\mathrm{S}$ & $\mathrm{P}$ & $\mathrm{P}$ & $\mathrm{P}$ & $\mathrm{P}$ \\
\hline Boraceia & $\mathrm{P}$ & $\mathrm{P}$ & $\mathrm{S}$ & $\mathrm{S}$ & $\mathrm{S}$ & $\mathrm{S}$ & $\mathrm{S}$ & $\mathrm{S}$ & $\mathrm{P}$ & $\mathrm{P}$ & $\mathrm{N}$ & $\mathrm{N}$ \\
\hline Borborema & $\mathrm{N}$ & $\mathrm{P}$ & $\mathrm{S}$ & $\mathrm{S}$ & $\mathrm{S}$ & $\mathrm{S}$ & $\mathrm{S}$ & $\mathrm{P}$ & $\mathrm{P}$ & $\mathrm{P}$ & $\mathrm{N}$ & $\mathrm{N}$ \\
\hline Borebi & $\mathrm{P}$ & $\mathrm{S}$ & $\mathrm{S}$ & NR & NR & NR & $\mathrm{S}$ & $\mathrm{S}$ & $\mathrm{S}$ & $\mathrm{P}$ & $\mathrm{P}$ & $\mathrm{P}$ \\
\hline Botucatu & $\mathrm{S}$ & $\mathrm{S}$ & NR & NR & NR & NR & NR & $\mathrm{S}$ & $\mathrm{S}$ & $\mathrm{S}$ & $\mathrm{S}$ & $\mathrm{S}$ \\
\hline
\end{tabular}


Tabela 10. Genótipos de milho mais recomendados para cada início de ciclo nos municípios do Estado de São Paulo, durante os doze meses do ano. N: normal; P: precoce; S: superprecoce; NR: não recomendável

\begin{tabular}{|c|c|c|c|c|c|c|c|c|c|c|c|c|}
\hline \multirow{2}{*}{ Município } & \multicolumn{12}{|c|}{ Meses } \\
\hline & Jan & $\mathrm{Fev}$ & Mar & Abr & Mai & Jun & Jul & Ago & Set & Out & Nov & Dez \\
\hline Bragança Paulista & $\mathrm{S}$ & $\mathrm{S}$ & $\mathrm{S}$ & NR & NR & NR & $\mathrm{S}$ & $\mathrm{S}$ & $\mathrm{S}$ & $\mathrm{S}$ & $\mathrm{P}$ & $\mathrm{P}$ \\
\hline Braúna & $\mathrm{P}$ & $\mathrm{P}$ & $\mathrm{S}$ & $\mathrm{S}$ & $\mathrm{S}$ & $\mathrm{S}$ & $\mathrm{S}$ & $\mathrm{S}$ & $\mathrm{P}$ & $\mathrm{P}$ & $\mathrm{P}$ & $\mathrm{P}$ \\
\hline Brejo Alegre & $\mathrm{N}$ & $\mathrm{P}$ & $\mathrm{S}$ & $\mathrm{S}$ & $\mathrm{S}$ & $\mathrm{S}$ & $\mathrm{S}$ & $\mathrm{P}$ & $\mathrm{P}$ & $\mathrm{N}$ & $\mathrm{N}$ & $\mathrm{N}$ \\
\hline Brodosqui & $\mathrm{P}$ & $\mathrm{S}$ & $\mathrm{S}$ & $\mathrm{S}$ & $\mathrm{S}$ & $\mathrm{S}$ & $\mathrm{S}$ & $\mathrm{S}$ & $\mathrm{P}$ & $\mathrm{P}$ & $\mathrm{P}$ & $\mathrm{P}$ \\
\hline Brotas & $\mathrm{P}$ & $\mathrm{S}$ & $\mathrm{S}$ & NR & NR & NR & $\mathrm{S}$ & $\mathrm{S}$ & $\mathrm{S}$ & $\mathrm{S}$ & $\mathrm{P}$ & $\mathrm{P}$ \\
\hline Buri & $\mathrm{S}$ & $\mathrm{S}$ & NR & NR & NR & NR & NR & $\mathrm{S}$ & $\mathrm{S}$ & $\mathrm{S}$ & $\mathrm{S}$ & $\mathrm{S}$ \\
\hline Buritama & $\mathrm{N}$ & $\mathrm{P}$ & $\mathrm{S}$ & $\mathrm{S}$ & $\mathrm{S}$ & $\mathrm{S}$ & $\mathrm{S}$ & $\mathrm{P}$ & $\mathrm{N}$ & $\mathrm{N}$ & $\mathrm{N}$ & $\mathrm{N}$ \\
\hline Buritizal & $\mathrm{S}$ & $\mathrm{S}$ & $\mathrm{S}$ & $\mathrm{S}$ & $\mathrm{S}$ & $\mathrm{S}$ & $\mathrm{S}$ & $\mathrm{S}$ & $\mathrm{S}$ & $\mathrm{P}$ & $\mathrm{P}$ & $\mathrm{P}$ \\
\hline Cabrália Paulista & $\mathrm{P}$ & $\mathrm{S}$ & $\mathrm{S}$ & NR & NR & $\mathrm{S}$ & $\mathrm{S}$ & $\mathrm{S}$ & $\mathrm{S}$ & $\mathrm{S}$ & $\mathrm{P}$ & $\mathrm{P}$ \\
\hline Cabreúva & $\mathrm{P}$ & $\mathrm{S}$ & $\mathrm{S}$ & NR & NR & NR & $\mathrm{S}$ & $\mathrm{S}$ & $\mathrm{S}$ & $\mathrm{P}$ & $\mathrm{P}$ & $\mathrm{P}$ \\
\hline Caçapava & $\mathrm{P}$ & $\mathrm{S}$ & $\mathrm{S}$ & NR & NR & NR & $\mathrm{S}$ & $\mathrm{S}$ & $\mathrm{S}$ & $\mathrm{S}$ & $\mathrm{P}$ & $\mathrm{P}$ \\
\hline Cachoeira Paulista & $\mathrm{P}$ & $\mathrm{S}$ & $\mathrm{S}$ & NR & NR & NR & $\mathrm{S}$ & $\mathrm{S}$ & $\mathrm{S}$ & $\mathrm{S}$ & $\mathrm{P}$ & $\mathrm{P}$ \\
\hline Caconde & $\mathrm{S}$ & $\mathrm{S}$ & NR & NR & NR & NR & $\mathrm{S}$ & $\mathrm{S}$ & $\mathrm{S}$ & $\mathrm{S}$ & $\mathrm{S}$ & $\mathrm{S}$ \\
\hline Cafelândia & $\mathrm{P}$ & $\mathrm{P}$ & $\mathrm{S}$ & $\mathrm{S}$ & $\mathrm{S}$ & $\mathrm{S}$ & $\mathrm{S}$ & $\mathrm{P}$ & $\mathrm{P}$ & $\mathrm{P}$ & $\mathrm{N}$ & $\mathrm{N}$ \\
\hline Caiabu & $\mathrm{N}$ & $\mathrm{P}$ & $\mathrm{S}$ & $\mathrm{S}$ & $\mathrm{S}$ & $\mathrm{S}$ & $\mathrm{S}$ & $\mathrm{P}$ & $\mathrm{P}$ & $\mathrm{N}$ & $\mathrm{N}$ & $\mathrm{N}$ \\
\hline Caieiras & $\mathrm{S}$ & $\mathrm{S}$ & NR & NR & NR & NR & NR & $\mathrm{S}$ & $\mathrm{S}$ & $\mathrm{S}$ & $\mathrm{S}$ & $\mathrm{S}$ \\
\hline Caiuá & $\mathrm{N}$ & $\mathrm{P}$ & $\mathrm{S}$ & $\mathrm{S}$ & $\mathrm{S}$ & $\mathrm{S}$ & $\mathrm{S}$ & $\mathrm{P}$ & $\mathrm{P}$ & $\mathrm{N}$ & $\mathrm{N}$ & $\mathrm{N}$ \\
\hline Cajamar & $\mathrm{S}$ & $\mathrm{S}$ & NR & NR & NR & NR & $\mathrm{S}$ & $\mathrm{S}$ & $\mathrm{S}$ & $\mathrm{S}$ & $\mathrm{P}$ & $\mathrm{P}$ \\
\hline Cajati & $\mathrm{S}$ & NR & NR & NR & NR & NR & NR & NR & $\mathrm{S}$ & $\mathrm{S}$ & $\mathrm{S}$ & $\mathrm{S}$ \\
\hline Cajobi & $\mathrm{P}$ & $\mathrm{S}$ & $\mathrm{S}$ & $\mathrm{S}$ & $\mathrm{S}$ & $\mathrm{S}$ & $\mathrm{S}$ & $\mathrm{S}$ & $\mathrm{P}$ & $\mathrm{P}$ & $\mathrm{P}$ & $\mathrm{P}$ \\
\hline Cajuru & $\mathrm{S}$ & $\mathrm{S}$ & NR & NR & NR & NR & $\mathrm{S}$ & $\mathrm{S}$ & $\mathrm{S}$ & $\mathrm{S}$ & $\mathrm{S}$ & $\mathrm{S}$ \\
\hline $\begin{array}{l}\text { Campina do Monte } \\
\text { Alegre }\end{array}$ & $\mathrm{S}$ & $\mathrm{S}$ & NR & NR & NR & NR & NR & $\mathrm{S}$ & $\mathrm{S}$ & $\mathrm{S}$ & $\mathrm{S}$ & $\mathrm{S}$ \\
\hline Campinas & $\mathrm{S}$ & NR & NR & NR & NR & NR & NR & NR & $\mathrm{S}$ & $\mathrm{S}$ & $\mathrm{S}$ & S \\
\hline Campo Limpo Paulista & $\mathrm{S}$ & $\mathrm{S}$ & NR & NR & NR & NR & NR & $\mathrm{S}$ & $\mathrm{S}$ & $\mathrm{S}$ & $\mathrm{S}$ & $\mathrm{S}$ \\
\hline Campos do Jordão & NR & NR & NR & NR & NR & NR & NR & NR & NR & NR & NR & NR \\
\hline Campos Novos Paulista & $\mathrm{P}$ & $\mathrm{S}$ & $\mathrm{S}$ & $\mathrm{S}$ & $\mathrm{S}$ & $\mathrm{S}$ & $\mathrm{S}$ & $\mathrm{S}$ & $\mathrm{S}$ & $\mathrm{P}$ & $\mathrm{P}$ & $\mathrm{P}$ \\
\hline Cananéia & $\mathrm{N}$ & $\mathrm{N}$ & $\mathrm{P}$ & $\mathrm{S}$ & $\mathrm{S}$ & $\mathrm{S}$ & $\mathrm{S}$ & $\mathrm{S}$ & $\mathrm{P}$ & $\mathrm{N}$ & $\mathrm{N}$ & $\mathrm{N}$ \\
\hline Canas & $\mathrm{S}$ & $\mathrm{NR}$ & NR & NR & NR & NR & NR & $\mathrm{S}$ & $\mathrm{S}$ & $\mathrm{S}$ & $\mathrm{S}$ & S \\
\hline Cândido Mota & $\mathrm{P}$ & $\mathrm{S}$ & $\mathrm{S}$ & $\mathrm{S}$ & NR & $\mathrm{S}$ & $\mathrm{S}$ & $\mathrm{S}$ & $\mathrm{S}$ & $\mathrm{P}$ & $\mathrm{P}$ & $\mathrm{P}$ \\
\hline Cândido Rodrigues & $\mathrm{P}$ & $\mathrm{P}$ & $\mathrm{S}$ & $\mathrm{S}$ & $\mathrm{S}$ & $\mathrm{S}$ & $\mathrm{S}$ & $\mathrm{P}$ & $\mathrm{P}$ & $\mathrm{P}$ & $\mathrm{P}$ & $\mathrm{P}$ \\
\hline Canitar & $\mathrm{P}$ & $\mathrm{S}$ & $\mathrm{S}$ & NR & NR & $\mathrm{S}$ & $\mathrm{S}$ & $\mathrm{S}$ & $\mathrm{S}$ & $\mathrm{P}$ & $\mathrm{P}$ & $\mathrm{P}$ \\
\hline Capão Bonito & $\mathrm{S}$ & $\mathrm{S}$ & NR & NR & NR & NR & NR & $\mathrm{S}$ & $\mathrm{S}$ & $\mathrm{S}$ & $\mathrm{S}$ & $\mathrm{S}$ \\
\hline Capela do Alto & $\mathrm{P}$ & $\mathrm{S}$ & $\mathrm{S}$ & NR & NR & NR & $\mathrm{S}$ & $\mathrm{S}$ & $\mathrm{S}$ & $\mathrm{S}$ & $\mathrm{P}$ & $\mathrm{P}$ \\
\hline Capivari & $\mathrm{P}$ & $\mathrm{S}$ & $\mathrm{S}$ & NR & NR & $\mathrm{S}$ & $\mathrm{S}$ & $\mathrm{S}$ & $\mathrm{S}$ & $\mathrm{P}$ & $\mathrm{P}$ & $\mathrm{P}$ \\
\hline Caraguatatuba & $\mathrm{N}$ & $\mathrm{N}$ & $\mathrm{P}$ & $\mathrm{S}$ & $\mathrm{S}$ & $\mathrm{S}$ & $\mathrm{S}$ & $\mathrm{P}$ & $\mathrm{N}$ & $\mathrm{N}$ & $\mathrm{N}$ & $\mathrm{N}$ \\
\hline Carapicuíba & $\mathrm{S}$ & $\mathrm{S}$ & NR & NR & NR & NR & $\mathrm{S}$ & $\mathrm{S}$ & $\mathrm{S}$ & $\mathrm{S}$ & $\mathrm{S}$ & $\mathrm{P}$ \\
\hline Cardoso & $\mathrm{N}$ & $\mathrm{P}$ & $\mathrm{S}$ & $\mathrm{S}$ & $\mathrm{S}$ & $\mathrm{S}$ & $\mathrm{P}$ & $\mathrm{P}$ & $\mathrm{N}$ & $\mathrm{N}$ & $\mathrm{N}$ & $\mathrm{N}$ \\
\hline Casa Branca & $\mathrm{S}$ & $\mathrm{S}$ & $\mathrm{S}$ & NR & NR & $\mathrm{S}$ & $\mathrm{S}$ & $\mathrm{S}$ & $\mathrm{S}$ & $\mathrm{S}$ & $\mathrm{P}$ & $\mathrm{S}$ \\
\hline Cássia dos Coqueiros & $\mathrm{S}$ & $\mathrm{S}$ & NR & NR & NR & NR & $\mathrm{S}$ & $\mathrm{S}$ & $\mathrm{S}$ & $\mathrm{S}$ & $\mathrm{S}$ & S \\
\hline Castilho & $\mathrm{N}$ & $\mathrm{N}$ & $\mathrm{P}$ & $\mathrm{S}$ & $\mathrm{S}$ & $\mathrm{S}$ & $\mathrm{P}$ & $\mathrm{P}$ & $\mathrm{N}$ & $\mathrm{N}$ & $\mathrm{N}$ & $\mathrm{N}$ \\
\hline Catanduva & $\mathrm{P}$ & $\mathrm{P}$ & $\mathrm{S}$ & $\mathrm{S}$ & $\mathrm{S}$ & $\mathrm{S}$ & $\mathrm{S}$ & $\mathrm{P}$ & $\mathrm{P}$ & $\mathrm{P}$ & $\mathrm{P}$ & $\mathrm{P}$ \\
\hline Catiguá & $\mathrm{P}$ & $\mathrm{P}$ & $\mathrm{S}$ & $\mathrm{S}$ & $\mathrm{S}$ & $\mathrm{S}$ & $\mathrm{S}$ & $\mathrm{P}$ & $\mathrm{P}$ & $\mathrm{P}$ & $\mathrm{P}$ & $\mathrm{N}$ \\
\hline
\end{tabular}


Tabela 10. Genótipos de milho mais recomendados para cada início de ciclo nos municípios do Estado de São Paulo, durante os doze meses do ano. N: normal; P: precoce; S: superprecoce; NR: não recomendável

\begin{tabular}{|c|c|c|c|c|c|c|c|c|c|c|c|c|}
\hline \multirow{2}{*}{ Município } & \multicolumn{12}{|c|}{ Meses } \\
\hline & Jan & Fev & Mar & Abr & Mai & Jun & Jul & Ago & Set & Out & Nov & Dez \\
\hline Cedral & $\mathrm{P}$ & $P$ & $\mathrm{~S}$ & $\mathrm{~S}$ & $\mathrm{~S}$ & $\mathrm{~S}$ & $\mathrm{~S}$ & $P$ & $\mathrm{P}$ & $\mathrm{P}$ & $\mathrm{N}$ & $\mathrm{N}$ \\
\hline Cerqueira César & $\mathrm{S}$ & $\mathrm{S}$ & NR & NR & NR & NR & NR & $\mathrm{S}$ & $\mathrm{S}$ & $\mathrm{S}$ & $\mathrm{S}$ & $\mathrm{S}$ \\
\hline Cerquilho & $\mathrm{P}$ & $\mathrm{S}$ & $\mathrm{S}$ & NR & NR & NR & $\mathrm{S}$ & $\mathrm{S}$ & $\mathrm{S}$ & $\mathrm{S}$ & $\mathrm{P}$ & $\mathrm{P}$ \\
\hline Cesário Lange & $\mathrm{S}$ & $\mathrm{S}$ & NR & NR & NR & NR & $\mathrm{S}$ & $\mathrm{S}$ & $\mathrm{S}$ & $\mathrm{S}$ & $\mathrm{P}$ & $\mathrm{P}$ \\
\hline Charqueada & $\mathrm{P}$ & $\mathrm{S}$ & $\mathrm{S}$ & NR & NR & NR & $\mathrm{S}$ & $\mathrm{S}$ & $\mathrm{S}$ & S & $\mathrm{P}$ & $\mathrm{P}$ \\
\hline Chavantes & $\mathrm{P}$ & $\mathrm{P}$ & $\mathrm{S}$ & $\mathrm{S}$ & $\mathrm{S}$ & $\mathrm{S}$ & $\mathrm{S}$ & $\mathrm{S}$ & $\mathrm{P}$ & $\mathrm{P}$ & $\mathrm{P}$ & $\mathrm{N}$ \\
\hline Clementina & $\mathrm{P}$ & $\mathrm{P}$ & S & $\mathrm{S}$ & $\mathrm{S}$ & $\mathrm{S}$ & S & $\mathrm{P}$ & $\mathrm{P}$ & $\mathrm{P}$ & $\mathrm{N}$ & $\mathrm{N}$ \\
\hline Colina & $\mathrm{P}$ & $\mathrm{S}$ & $\mathrm{S}$ & $\mathrm{S}$ & $\mathrm{S}$ & $\mathrm{S}$ & $\mathrm{S}$ & $\mathrm{S}$ & $\mathrm{P}$ & $\mathrm{P}$ & $\mathrm{P}$ & $\mathrm{P}$ \\
\hline Colômbia & $\mathrm{N}$ & $\mathrm{P}$ & $\mathrm{S}$ & $\mathrm{S}$ & $\mathrm{S}$ & $\mathrm{S}$ & $\mathrm{P}$ & $\mathrm{P}$ & $\mathrm{N}$ & $\mathrm{N}$ & $\mathrm{N}$ & $\mathrm{N}$ \\
\hline Conchal & $\mathrm{S}$ & $\mathrm{S}$ & $\mathrm{S}$ & NR & NR & $\mathrm{S}$ & $\mathrm{S}$ & $\mathrm{S}$ & $\mathrm{S}$ & $\mathrm{S}$ & $\mathrm{P}$ & $\mathrm{P}$ \\
\hline Conchas & $\mathrm{P}$ & $\mathrm{S}$ & $\mathrm{S}$ & NR & NR & $\mathrm{S}$ & $\mathrm{S}$ & $\mathrm{S}$ & $\mathrm{S}$ & $\mathrm{P}$ & $\mathrm{P}$ & $\mathrm{P}$ \\
\hline Cordeirópolis & $\mathrm{S}$ & S & NR & NR & NR & NR & NR & S & $\mathrm{S}$ & $\mathrm{S}$ & S & S \\
\hline Coroados & $\mathrm{N}$ & $\mathrm{P}$ & S & $\mathrm{S}$ & $\mathrm{S}$ & $\mathrm{S}$ & S & $\mathrm{P}$ & $\mathrm{P}$ & $\mathrm{N}$ & $\mathrm{N}$ & $\mathrm{N}$ \\
\hline Coronel Macedo & $\mathrm{S}$ & $\mathrm{S}$ & NR & NR & NR & NR & NR & $\mathrm{S}$ & $\mathrm{S}$ & $\mathrm{S}$ & $\mathrm{P}$ & $\mathrm{P}$ \\
\hline Corumbataí & $\mathrm{S}$ & $\mathrm{S}$ & NR & NR & $\mathrm{S}$ & $\mathrm{S}$ & $\mathrm{S}$ & $\mathrm{S}$ & $\mathrm{S}$ & $\mathrm{P}$ & $\mathrm{P}$ & $\mathrm{P}$ \\
\hline Cosmópolis & $\mathrm{P}$ & $\mathrm{S}$ & $\mathrm{S}$ & NR & NR & $\mathrm{S}$ & $\mathrm{S}$ & $\mathrm{S}$ & $\mathrm{S}$ & $\mathrm{P}$ & $\mathrm{P}$ & $\mathrm{P}$ \\
\hline Cosmorama & $\mathrm{P}$ & $\mathrm{P}$ & $\mathrm{S}$ & $\mathrm{S}$ & $\mathrm{S}$ & $\mathrm{S}$ & $\mathrm{S}$ & $\mathrm{P}$ & $\mathrm{P}$ & $\mathrm{N}$ & $\mathrm{N}$ & $\mathrm{N}$ \\
\hline Cotia & $\mathrm{S}$ & NR & NR & NR & NR & NR & NR & NR & $\mathrm{S}$ & $\mathrm{S}$ & $\mathrm{S}$ & $\mathrm{S}$ \\
\hline Cravinhos & $\mathrm{S}$ & $\mathrm{S}$ & NR & NR & NR & NR & $\mathrm{S}$ & S & $\mathrm{S}$ & $\mathrm{S}$ & S & S \\
\hline Cristais Paulista & $\mathrm{P}$ & $\mathrm{S}$ & $\mathrm{S}$ & $\mathrm{S}$ & $\mathrm{S}$ & $\mathrm{S}$ & $\mathrm{S}$ & $\mathrm{P}$ & $\mathrm{P}$ & $\mathrm{P}$ & $\mathrm{P}$ & $\mathrm{P}$ \\
\hline Cruzália & $\mathrm{P}$ & $\mathrm{P}$ & $\mathrm{S}$ & $\mathrm{S}$ & $\mathrm{S}$ & $\mathrm{S}$ & $\mathrm{S}$ & $\mathrm{S}$ & $\mathrm{P}$ & $\mathrm{P}$ & $\mathrm{P}$ & $\mathrm{N}$ \\
\hline Cruzeiro & $\mathrm{P}$ & $\mathrm{S}$ & $\mathrm{S}$ & NR & NR & $\mathrm{S}$ & $\mathrm{S}$ & $\mathrm{S}$ & $\mathrm{S}$ & $\mathrm{P}$ & $\mathrm{P}$ & $\mathrm{P}$ \\
\hline Cubatão & $\mathrm{N}$ & $\mathrm{N}$ & $\mathrm{P}$ & $\mathrm{S}$ & $\mathrm{S}$ & $\mathrm{S}$ & $\mathrm{S}$ & $\mathrm{P}$ & $\mathrm{N}$ & $\mathrm{N}$ & $\mathrm{N}$ & $\mathrm{N}$ \\
\hline Cunha & $\mathrm{S}$ & NR & NR & NR & NR & NR & NR & $\mathrm{S}$ & $\mathrm{S}$ & $\mathrm{S}$ & $\mathrm{S}$ & $\mathrm{S}$ \\
\hline Descalvado & $\mathrm{S}$ & S & S & NR & NR & $\mathrm{S}$ & S & S & $\mathrm{S}$ & $\mathrm{S}$ & $\mathrm{P}$ & $\mathrm{P}$ \\
\hline Diadema & $\mathrm{S}$ & $\mathrm{S}$ & NR & NR & NR & NR & NR & $\mathrm{S}$ & $\mathrm{S}$ & $\mathrm{S}$ & $\mathrm{S}$ & $\mathrm{S}$ \\
\hline Dirce Reis & $\mathrm{N}$ & $\mathrm{P}$ & $\mathrm{P}$ & $\mathrm{S}$ & $\mathrm{S}$ & $\mathrm{S}$ & $\mathrm{P}$ & $\mathrm{P}$ & $\mathrm{N}$ & $\mathrm{N}$ & $\mathrm{N}$ & $\mathrm{N}$ \\
\hline Divinolândia & $\mathrm{P}$ & $\mathrm{S}$ & $\mathrm{S}$ & $\mathrm{S}$ & NR & $\mathrm{S}$ & $\mathrm{S}$ & $\mathrm{S}$ & $\mathrm{S}$ & $\mathrm{S}$ & $\mathrm{P}$ & $\mathrm{P}$ \\
\hline Dobrada & $\mathrm{P}$ & $\mathrm{S}$ & $\mathrm{S}$ & $\mathrm{S}$ & $\mathrm{S}$ & $\mathrm{S}$ & $\mathrm{S}$ & $\mathrm{S}$ & $\mathrm{P}$ & $\mathrm{P}$ & $P$ & $\mathrm{P}$ \\
\hline Dois Córregos & $\mathrm{S}$ & $\mathrm{S}$ & NR & NR & NR & NR & $\mathrm{S}$ & $\mathrm{S}$ & $\mathrm{S}$ & $\mathrm{S}$ & $\mathrm{S}$ & $\mathrm{P}$ \\
\hline Dolcinópolis & $\mathrm{N}$ & $\mathrm{P}$ & $\mathrm{P}$ & S & $\mathrm{S}$ & $\mathrm{S}$ & $\mathrm{P}$ & $\mathrm{N}$ & $\mathrm{N}$ & $\mathrm{N}$ & $\mathrm{N}$ & $\mathrm{N}$ \\
\hline Dourado & $\mathrm{S}$ & $\mathrm{S}$ & NR & NR & NR & NR & $\mathrm{S}$ & $\mathrm{S}$ & $\mathrm{S}$ & $\mathrm{S}$ & $\mathrm{S}$ & $\mathrm{S}$ \\
\hline Dracena & $\mathrm{N}$ & $\mathrm{P}$ & $\mathrm{S}$ & $\mathrm{S}$ & $\mathrm{S}$ & $\mathrm{S}$ & $\mathrm{S}$ & $\mathrm{P}$ & $\mathrm{P}$ & $\mathrm{P}$ & $\mathrm{N}$ & $\mathrm{N}$ \\
\hline Duartina & $\mathrm{P}$ & S & S & $\mathrm{S}$ & NR & $\mathrm{S}$ & S & S & $\mathrm{S}$ & $\mathrm{P}$ & $\mathrm{P}$ & $\mathrm{P}$ \\
\hline Dumont & $\mathrm{P}$ & $\mathrm{S}$ & $\mathrm{S}$ & $\mathrm{S}$ & $\mathrm{S}$ & $\mathrm{S}$ & $\mathrm{S}$ & $\mathrm{S}$ & $\mathrm{P}$ & $\mathrm{P}$ & $\mathrm{P}$ & $\mathrm{P}$ \\
\hline Echapora & $\mathrm{P}$ & S & S & $\mathrm{S}$ & $\mathrm{S}$ & $\mathrm{S}$ & S & S & $\mathrm{S}$ & $\mathrm{P}$ & $\mathrm{P}$ & $\mathrm{P}$ \\
\hline Eldorado & $\mathrm{N}$ & $\mathrm{P}$ & S & $\mathrm{S}$ & S & $\mathrm{S}$ & S & S & $\mathrm{P}$ & $\mathrm{N}$ & $\mathrm{N}$ & $\mathrm{N}$ \\
\hline Elias Fausto & $\mathrm{P}$ & $\mathrm{S}$ & S & NR & NR & NR & $\mathrm{S}$ & $\mathrm{S}$ & $\mathrm{S}$ & $\mathrm{S}$ & $\mathrm{P}$ & $\mathrm{P}$ \\
\hline Elisiário & $\mathrm{P}$ & $\mathrm{P}$ & $\mathrm{S}$ & $\mathrm{S}$ & $\mathrm{S}$ & $\mathrm{S}$ & $\mathrm{S}$ & $\mathrm{P}$ & $\mathrm{P}$ & $\mathrm{P}$ & $\mathrm{P}$ & $\mathrm{P}$ \\
\hline Embaúba & $\mathrm{P}$ & $\mathrm{P}$ & $\mathrm{S}$ & $\mathrm{S}$ & S & $\mathrm{S}$ & $\mathrm{S}$ & $\mathrm{P}$ & $\mathrm{P}$ & $\mathrm{P}$ & $\mathrm{P}$ & $\mathrm{P}$ \\
\hline Embu & $\mathrm{S}$ & $\mathrm{S}$ & NR & NR & NR & NR & $\mathrm{S}$ & $\mathrm{S}$ & $\mathrm{S}$ & $\mathrm{S}$ & $\mathrm{S}$ & $\mathrm{P}$ \\
\hline Embu-Guaçu & $\mathrm{S}$ & $\mathrm{S}$ & NR & NR & NR & NR & NR & $\mathrm{S}$ & $\mathrm{S}$ & $\mathrm{S}$ & $\mathrm{S}$ & $\mathrm{S}$ \\
\hline Emilianópolis & $\mathrm{N}$ & $\mathrm{P}$ & $\mathrm{S}$ & $\mathrm{S}$ & $\mathrm{S}$ & $\mathrm{S}$ & $\mathrm{S}$ & $\mathrm{P}$ & $\mathrm{N}$ & $\mathrm{N}$ & $\mathrm{N}$ & $\mathrm{N}$ \\
\hline
\end{tabular}


Tabela 10. Genótipos de milho mais recomendados para cada início de ciclo nos municípios do Estado de São Paulo, durante os doze meses do ano. N: normal; P: precoce; S: superprecoce; NR: não recomendável

\begin{tabular}{|c|c|c|c|c|c|c|c|c|c|c|c|c|}
\hline \multirow{2}{*}{ Município } & \multicolumn{12}{|c|}{ Meses } \\
\hline & Jan & Fev & Mar & Abr & Mai & Jun & Jul & Ago & Set & Out & Nov & Dez \\
\hline Engenheiro Coelho & $\mathrm{S}$ & $\mathrm{S}$ & NR & NR & NR & $\mathrm{S}$ & $\mathrm{S}$ & $\mathrm{S}$ & $\mathrm{S}$ & $\mathrm{S}$ & $\mathrm{P}$ & $\mathrm{P}$ \\
\hline Espírito Santo do Pinhal & $\mathrm{S}$ & NR & NR & NR & NR & NR & NR & $\mathrm{S}$ & $\mathrm{S}$ & $\mathrm{S}$ & $\mathrm{S}$ & $\mathrm{S}$ \\
\hline Espírito Santo do Turvo & $\mathrm{P}$ & $\mathrm{S}$ & $\mathrm{S}$ & NR & NR & NR & $\mathrm{S}$ & $\mathrm{S}$ & $\mathrm{S}$ & $\mathrm{S}$ & $\mathrm{P}$ & $\mathrm{P}$ \\
\hline Estiva Gerbi & $\mathrm{P}$ & $\mathrm{S}$ & $\mathrm{S}$ & NR & NR & $\mathrm{S}$ & $\mathrm{S}$ & $\mathrm{S}$ & $\mathrm{S}$ & $\mathrm{S}$ & $\mathrm{P}$ & $\mathrm{P}$ \\
\hline Estrela do Norte & $\mathrm{N}$ & $\mathrm{P}$ & $\mathrm{S}$ & $\mathrm{S}$ & $\mathrm{S}$ & $\mathrm{S}$ & $\mathrm{S}$ & $\mathrm{P}$ & $\mathrm{P}$ & $\mathrm{N}$ & $\mathrm{N}$ & $\mathrm{N}$ \\
\hline Estrela D'oeste & $\mathrm{N}$ & $\mathrm{P}$ & $\mathrm{P}$ & $\mathrm{S}$ & $\mathrm{S}$ & $\mathrm{S}$ & $P$ & $\mathrm{~N}$ & $\mathrm{~N}$ & $\mathrm{~N}$ & $\mathrm{~N}$ & $\mathrm{~N}$ \\
\hline $\begin{array}{l}\text { Euclides da Cunha } \\
\text { Paulista }\end{array}$ & $\mathrm{P}$ & $\mathrm{P}$ & $\mathrm{S}$ & $\mathrm{S}$ & $\mathrm{S}$ & $\mathrm{S}$ & $\mathrm{S}$ & $\mathrm{S}$ & $\mathrm{P}$ & $\mathrm{P}$ & $\mathrm{N}$ & $\mathrm{N}$ \\
\hline Fartura & $\mathrm{P}$ & $\mathrm{S}$ & NR & NR & NR & NR & $\mathrm{S}$ & $\mathrm{S}$ & $\mathrm{S}$ & $\mathrm{S}$ & $\mathrm{P}$ & $\mathrm{P}$ \\
\hline Fernando Prestes & $\mathrm{P}$ & $\mathrm{P}$ & $\mathrm{S}$ & $\mathrm{S}$ & $\mathrm{S}$ & $\mathrm{S}$ & $\mathrm{S}$ & $\mathrm{P}$ & $\mathrm{P}$ & $\mathrm{P}$ & $\mathrm{P}$ & $\mathrm{P}$ \\
\hline Fernandópolis & $\mathrm{P}$ & $\mathrm{P}$ & $\mathrm{S}$ & $\mathrm{S}$ & $\mathrm{S}$ & $\mathrm{S}$ & $\mathrm{S}$ & $\mathrm{P}$ & $\mathrm{P}$ & $\mathrm{P}$ & $\mathrm{P}$ & $\mathrm{P}$ \\
\hline Fernão & $\mathrm{P}$ & $\mathrm{S}$ & $\mathrm{S}$ & $\mathrm{S}$ & $\mathrm{S}$ & $\mathrm{S}$ & $\mathrm{S}$ & $\mathrm{S}$ & $\mathrm{P}$ & $\mathrm{P}$ & $\mathrm{P}$ & $\mathrm{P}$ \\
\hline Ferraz de Vasconcelos & $\mathrm{S}$ & $\mathrm{S}$ & NR & NR & NR & NR & NR & $\mathrm{S}$ & $\mathrm{S}$ & $\mathrm{S}$ & $\mathrm{S}$ & $\mathrm{S}$ \\
\hline Flora Rica & $\mathrm{N}$ & $\mathrm{P}$ & $\mathrm{P}$ & $\mathrm{S}$ & $\mathrm{S}$ & $\mathrm{S}$ & $\mathrm{S}$ & $\mathrm{P}$ & $\mathrm{N}$ & $\mathrm{N}$ & $\mathrm{N}$ & $\mathrm{N}$ \\
\hline Floreal & $\mathrm{N}$ & $\mathrm{P}$ & $\mathrm{S}$ & $\mathrm{S}$ & $\mathrm{S}$ & $\mathrm{S}$ & $\mathrm{P}$ & $\mathrm{P}$ & $\mathrm{N}$ & $\mathrm{N}$ & $\mathrm{N}$ & $\mathrm{N}$ \\
\hline Florida Paulista & $\mathrm{P}$ & $\mathrm{P}$ & $\mathrm{S}$ & $\mathrm{S}$ & $\mathrm{S}$ & $\mathrm{S}$ & $\mathrm{S}$ & $\mathrm{P}$ & $\mathrm{P}$ & $\mathrm{P}$ & $\mathrm{N}$ & $\mathrm{N}$ \\
\hline Florínia & $\mathrm{P}$ & $\mathrm{P}$ & $\mathrm{S}$ & $\mathrm{S}$ & $\mathrm{S}$ & $\mathrm{S}$ & $\mathrm{S}$ & $\mathrm{S}$ & $\mathrm{P}$ & $\mathrm{P}$ & $\mathrm{N}$ & $\mathrm{N}$ \\
\hline Franca & $\mathrm{S}$ & NR & NR & NR & NR & NR & NR & $\mathrm{S}$ & $\mathrm{S}$ & $\mathrm{S}$ & $\mathrm{S}$ & $\mathrm{S}$ \\
\hline Francisco Morato & $\mathrm{S}$ & NR & NR & NR & NR & NR & NR & $\mathrm{S}$ & $\mathrm{S}$ & $\mathrm{S}$ & $\mathrm{S}$ & $\mathrm{S}$ \\
\hline Franco da Rocha & $\mathrm{S}$ & $\mathrm{S}$ & NR & NR & NR & NR & NR & $\mathrm{S}$ & $\mathrm{S}$ & $\mathrm{S}$ & $\mathrm{S}$ & $\mathrm{S}$ \\
\hline Gabriel Monteiro & $\mathrm{P}$ & $\mathrm{P}$ & $\mathrm{S}$ & $\mathrm{S}$ & $\mathrm{S}$ & $\mathrm{S}$ & $\mathrm{S}$ & $\mathrm{P}$ & $\mathrm{P}$ & $\mathrm{P}$ & $\mathrm{N}$ & $\mathrm{N}$ \\
\hline Gália & $\mathrm{P}$ & $\mathrm{S}$ & NR & NR & NR & NR & $\mathrm{S}$ & $\mathrm{S}$ & $\mathrm{S}$ & $\mathrm{P}$ & $\mathrm{P}$ & $\mathrm{P}$ \\
\hline Garça & $\mathrm{S}$ & $\mathrm{S}$ & $\mathrm{S}$ & NR & NR & NR & $\mathrm{S}$ & $\mathrm{S}$ & $\mathrm{S}$ & $\mathrm{S}$ & $\mathrm{S}$ & $\mathrm{P}$ \\
\hline Gastão Vidigal & $\mathrm{N}$ & $\mathrm{P}$ & $\mathrm{S}$ & $\mathrm{S}$ & $\mathrm{S}$ & $\mathrm{S}$ & $\mathrm{P}$ & $\mathrm{P}$ & $\mathrm{N}$ & $\mathrm{N}$ & $\mathrm{N}$ & $\mathrm{N}$ \\
\hline Gavião Peixoto & $\mathrm{P}$ & $\mathrm{P}$ & $\mathrm{S}$ & $\mathrm{S}$ & $\mathrm{S}$ & $\mathrm{S}$ & $\mathrm{S}$ & $\mathrm{S}$ & $\mathrm{P}$ & $\mathrm{P}$ & $\mathrm{P}$ & $\mathrm{P}$ \\
\hline General Salgado & $\mathrm{N}$ & $\mathrm{P}$ & $\mathrm{P}$ & $\mathrm{S}$ & $\mathrm{S}$ & $\mathrm{S}$ & $\mathrm{P}$ & $\mathrm{P}$ & $\mathrm{N}$ & $\mathrm{N}$ & $\mathrm{N}$ & $\mathrm{N}$ \\
\hline Getulina & $\mathrm{P}$ & $\mathrm{P}$ & $\mathrm{S}$ & $\mathrm{S}$ & $\mathrm{S}$ & $\mathrm{S}$ & $\mathrm{S}$ & $\mathrm{S}$ & $\mathrm{P}$ & $\mathrm{P}$ & $\mathrm{P}$ & $\mathrm{N}$ \\
\hline Glicério & $\mathrm{N}$ & $\mathrm{P}$ & $\mathrm{S}$ & $\mathrm{S}$ & $\mathrm{S}$ & $\mathrm{S}$ & $\mathrm{S}$ & $\mathrm{P}$ & $\mathrm{P}$ & $\mathrm{N}$ & $\mathrm{N}$ & $\mathrm{N}$ \\
\hline Guaiçara & $\mathrm{P}$ & $\mathrm{P}$ & $\mathrm{S}$ & $\mathrm{S}$ & $\mathrm{S}$ & $\mathrm{S}$ & $\mathrm{S}$ & $\mathrm{P}$ & $\mathrm{P}$ & $\mathrm{P}$ & $\mathrm{P}$ & $\mathrm{N}$ \\
\hline Guaimbé & $\mathrm{P}$ & $\mathrm{P}$ & $\mathrm{S}$ & $\mathrm{S}$ & $\mathrm{S}$ & $\mathrm{S}$ & $\mathrm{S}$ & $\mathrm{S}$ & $\mathrm{P}$ & $\mathrm{P}$ & $\mathrm{P}$ & $\mathrm{N}$ \\
\hline Guaíra & $\mathrm{N}$ & $\mathrm{P}$ & $\mathrm{S}$ & $\mathrm{S}$ & $\mathrm{S}$ & $\mathrm{S}$ & $\mathrm{S}$ & $\mathrm{P}$ & $\mathrm{N}$ & $\mathrm{N}$ & $\mathrm{N}$ & $\mathrm{N}$ \\
\hline Guapiaçu & $\mathrm{P}$ & $\mathrm{P}$ & $\mathrm{S}$ & $\mathrm{S}$ & $\mathrm{S}$ & $\mathrm{S}$ & $\mathrm{S}$ & $\mathrm{P}$ & $\mathrm{P}$ & $\mathrm{P}$ & $\mathrm{P}$ & $\mathrm{N}$ \\
\hline Guapiara & $\mathrm{S}$ & $\mathrm{S}$ & $\mathrm{S}$ & NR & NR & $\mathrm{S}$ & $\mathrm{S}$ & $\mathrm{S}$ & $\mathrm{S}$ & $\mathrm{S}$ & $\mathrm{S}$ & $\mathrm{S}$ \\
\hline Guará & $\mathrm{P}$ & $\mathrm{S}$ & $\mathrm{S}$ & $\mathrm{S}$ & $\mathrm{S}$ & $\mathrm{S}$ & $\mathrm{S}$ & $\mathrm{P}$ & $\mathrm{P}$ & $\mathrm{P}$ & $\mathrm{P}$ & $\mathrm{P}$ \\
\hline Guaraçaí & $\mathrm{N}$ & $\mathrm{P}$ & $\mathrm{P}$ & $\mathrm{S}$ & $\mathrm{S}$ & $\mathrm{S}$ & $\mathrm{S}$ & $\mathrm{P}$ & $\mathrm{N}$ & $\mathrm{N}$ & $\mathrm{N}$ & $\mathrm{N}$ \\
\hline Guaraci & $\mathrm{N}$ & $\mathrm{P}$ & $\mathrm{S}$ & $\mathrm{S}$ & $\mathrm{S}$ & $\mathrm{S}$ & $\mathrm{S}$ & $\mathrm{P}$ & $\mathrm{N}$ & $\mathrm{N}$ & $\mathrm{N}$ & $\mathrm{N}$ \\
\hline Guarani D'oeste & $\mathrm{N}$ & $\mathrm{P}$ & $\mathrm{P}$ & $\mathrm{S}$ & $\mathrm{S}$ & $\mathrm{S}$ & $\mathrm{P}$ & $\mathrm{N}$ & $\mathrm{N}$ & $\mathrm{N}$ & $\mathrm{N}$ & $\mathrm{N}$ \\
\hline Guarantã & $\mathrm{P}$ & $\mathrm{S}$ & $\mathrm{S}$ & $\mathrm{S}$ & $\mathrm{S}$ & $\mathrm{S}$ & $\mathrm{S}$ & $\mathrm{S}$ & $\mathrm{P}$ & $\mathrm{P}$ & $\mathrm{P}$ & $\mathrm{P}$ \\
\hline Guararapes & $\mathrm{N}$ & $\mathrm{P}$ & $\mathrm{S}$ & $\mathrm{S}$ & $\mathrm{S}$ & $\mathrm{S}$ & $\mathrm{S}$ & $\mathrm{P}$ & $\mathrm{P}$ & $\mathrm{N}$ & $\mathrm{N}$ & $\mathrm{N}$ \\
\hline Guararema & $\mathrm{S}$ & $\mathrm{S}$ & NR & NR & NR & NR & $\mathrm{S}$ & $\mathrm{S}$ & $\mathrm{S}$ & $\mathrm{S}$ & $\mathrm{P}$ & $\mathrm{P}$ \\
\hline Guaratinguetá & $\mathrm{P}$ & $\mathrm{S}$ & $\mathrm{S}$ & NR & NR & $\mathrm{S}$ & $\mathrm{S}$ & $\mathrm{S}$ & $\mathrm{S}$ & $\mathrm{P}$ & $\mathrm{P}$ & $\mathrm{P}$ \\
\hline Guareí & $\mathrm{S}$ & $\mathrm{S}$ & NR & NR & NR & NR & NR & $\mathrm{S}$ & $\mathrm{S}$ & $\mathrm{S}$ & $\mathrm{S}$ & $\mathrm{S}$ \\
\hline Guariba & $\mathrm{P}$ & $\mathrm{S}$ & $\mathrm{S}$ & $\mathrm{S}$ & $\mathrm{S}$ & $\mathrm{S}$ & $\mathrm{S}$ & $\mathrm{S}$ & $\mathrm{S}$ & $\mathrm{P}$ & $\mathrm{P}$ & $\mathrm{P}$ \\
\hline
\end{tabular}


Tabela 10. Genótipos de milho mais recomendados para cada início de ciclo nos municípios do Estado de São Paulo, durante os doze meses do ano. N: normal; P: precoce; S: superprecoce; NR: não recomendável

\begin{tabular}{|c|c|c|c|c|c|c|c|c|c|c|c|c|}
\hline \multirow{2}{*}{ Município } & \multicolumn{12}{|c|}{ Meses } \\
\hline & Jan & $\mathrm{Fev}$ & Mar & Abr & Mai & Jun & Jul & Ago & Set & Out & Nov & Dez \\
\hline Guarujá & $\mathrm{N}$ & $\mathrm{N}$ & $\mathrm{P}$ & $\mathrm{S}$ & $\mathrm{S}$ & $\mathrm{S}$ & $\mathrm{S}$ & $\mathrm{P}$ & $\mathrm{N}$ & $\mathrm{N}$ & $\mathrm{N}$ & $\mathrm{N}$ \\
\hline Guarulhos & $\mathrm{S}$ & $\mathrm{S}$ & NR & NR & NR & NR & NR & $\mathrm{S}$ & $\mathrm{S}$ & $\mathrm{S}$ & $\mathrm{S}$ & $\mathrm{S}$ \\
\hline Guatapara & $\mathrm{P}$ & $\mathrm{S}$ & $\mathrm{S}$ & $\mathrm{S}$ & $\mathrm{S}$ & $\mathrm{S}$ & $\mathrm{S}$ & $\mathrm{S}$ & $\mathrm{S}$ & $\mathrm{P}$ & $\mathrm{P}$ & $\mathrm{P}$ \\
\hline Guzolândia & $\mathrm{N}$ & $\mathrm{P}$ & $\mathrm{S}$ & $\mathrm{S}$ & $\mathrm{S}$ & $\mathrm{S}$ & $\mathrm{S}$ & $\mathrm{P}$ & $\mathrm{N}$ & $\mathrm{N}$ & $\mathrm{N}$ & $\mathrm{N}$ \\
\hline Herculândia & $\mathrm{P}$ & $\mathrm{P}$ & $\mathrm{S}$ & $\mathrm{S}$ & $\mathrm{S}$ & $\mathrm{S}$ & $\mathrm{S}$ & $\mathrm{S}$ & $\mathrm{P}$ & $\mathrm{P}$ & $\mathrm{P}$ & $\mathrm{N}$ \\
\hline Holambra & $\mathrm{S}$ & $\mathrm{S}$ & $\mathrm{S}$ & NR & NR & $\mathrm{S}$ & $\mathrm{S}$ & $\mathrm{S}$ & $\mathrm{S}$ & $\mathrm{S}$ & $\mathrm{P}$ & $\mathrm{P}$ \\
\hline Hortolândia & $\mathrm{S}$ & $\mathrm{S}$ & $\mathrm{S}$ & NR & NR & NR & $\mathrm{S}$ & $\mathrm{S}$ & $\mathrm{S}$ & $\mathrm{S}$ & $\mathrm{P}$ & $\mathrm{P}$ \\
\hline Iacanga & $\mathrm{P}$ & $\mathrm{P}$ & $\mathrm{S}$ & $\mathrm{S}$ & $\mathrm{S}$ & $\mathrm{S}$ & $\mathrm{S}$ & $\mathrm{S}$ & $\mathrm{P}$ & $\mathrm{P}$ & $\mathrm{P}$ & $\mathrm{P}$ \\
\hline Iacri & $\mathrm{P}$ & $\mathrm{S}$ & $\mathrm{S}$ & $\mathrm{S}$ & $\mathrm{S}$ & $\mathrm{S}$ & $\mathrm{S}$ & $\mathrm{S}$ & $\mathrm{P}$ & $\mathrm{P}$ & $\mathrm{P}$ & $\mathrm{P}$ \\
\hline Iaras & $\mathrm{P}$ & $\mathrm{S}$ & $\mathrm{S}$ & NR & NR & NR & $\mathrm{S}$ & $\mathrm{S}$ & $\mathrm{S}$ & $\mathrm{P}$ & $\mathrm{P}$ & $\mathrm{P}$ \\
\hline Ibaté & $\mathrm{P}$ & $\mathrm{S}$ & $\mathrm{S}$ & $\mathrm{S}$ & $\mathrm{S}$ & $\mathrm{S}$ & $\mathrm{S}$ & $\mathrm{S}$ & $\mathrm{P}$ & $\mathrm{P}$ & $\mathrm{P}$ & $\mathrm{P}$ \\
\hline Ibira & $\mathrm{P}$ & $\mathrm{P}$ & $\mathrm{S}$ & $\mathrm{S}$ & $\mathrm{S}$ & $\mathrm{S}$ & $\mathrm{S}$ & $\mathrm{P}$ & $\mathrm{P}$ & $\mathrm{P}$ & $\mathrm{N}$ & $\mathrm{N}$ \\
\hline Ibirarema & $\mathrm{P}$ & $\mathrm{S}$ & $\mathrm{S}$ & $\mathrm{S}$ & $\mathrm{S}$ & $\mathrm{S}$ & $\mathrm{S}$ & $\mathrm{S}$ & $\mathrm{S}$ & $\mathrm{P}$ & $\mathrm{P}$ & $\mathrm{P}$ \\
\hline Ibitinga & $\mathrm{P}$ & $\mathrm{P}$ & $\mathrm{S}$ & $\mathrm{S}$ & $\mathrm{S}$ & $\mathrm{S}$ & $\mathrm{S}$ & $\mathrm{S}$ & $\mathrm{P}$ & $\mathrm{P}$ & $\mathrm{P}$ & $\mathrm{N}$ \\
\hline Ibiúna & $\mathrm{S}$ & NR & NR & NR & NR & NR & NR & NR & $\mathrm{S}$ & $\mathrm{S}$ & $\mathrm{S}$ & $\mathrm{S}$ \\
\hline Icém & $\mathrm{N}$ & $\mathrm{P}$ & $\mathrm{S}$ & $\mathrm{S}$ & $\mathrm{S}$ & $\mathrm{S}$ & $\mathrm{P}$ & $\mathrm{P}$ & $\mathrm{N}$ & $\mathrm{N}$ & $\mathrm{N}$ & $\mathrm{N}$ \\
\hline Iepe & $\mathrm{P}$ & $\mathrm{P}$ & $\mathrm{S}$ & $\mathrm{S}$ & $\mathrm{S}$ & $\mathrm{S}$ & $\mathrm{S}$ & $\mathrm{S}$ & $\mathrm{P}$ & $\mathrm{P}$ & $\mathrm{N}$ & $\mathrm{N}$ \\
\hline Igaraçu do Tietê & $\mathrm{P}$ & $\mathrm{S}$ & $\mathrm{S}$ & $\mathrm{S}$ & NR & $\mathrm{S}$ & $\mathrm{S}$ & $\mathrm{S}$ & $\mathrm{S}$ & $\mathrm{P}$ & $\mathrm{P}$ & $\mathrm{P}$ \\
\hline Igarapava & $\mathrm{P}$ & $\mathrm{S}$ & $\mathrm{S}$ & $\mathrm{S}$ & $\mathrm{S}$ & $\mathrm{S}$ & $\mathrm{S}$ & $\mathrm{P}$ & $\mathrm{P}$ & $\mathrm{P}$ & $\mathrm{P}$ & $\mathrm{P}$ \\
\hline Igaratá & $\mathrm{S}$ & NR & NR & NR & NR & NR & NR & NR & $\mathrm{S}$ & $\mathrm{S}$ & $\mathrm{S}$ & $\mathrm{S}$ \\
\hline Iguape & $\mathrm{P}$ & $\mathrm{S}$ & $\mathrm{S}$ & NR & NR & NR & NR & $\mathrm{S}$ & $\mathrm{S}$ & $\mathrm{S}$ & $\mathrm{P}$ & $\mathrm{P}$ \\
\hline Ilha Comprida & $\mathrm{N}$ & $\mathrm{P}$ & $\mathrm{S}$ & $\mathrm{S}$ & NR & $\mathrm{S}$ & $\mathrm{S}$ & $\mathrm{S}$ & $\mathrm{S}$ & $\mathrm{P}$ & $\mathrm{N}$ & $\mathrm{N}$ \\
\hline Ilha Solteira & $\mathrm{N}$ & $\mathrm{N}$ & $\mathrm{P}$ & $\mathrm{S}$ & $\mathrm{S}$ & $\mathrm{S}$ & $P$ & $\mathrm{~N}$ & $\mathrm{~N}$ & $\mathrm{~N}$ & $\mathrm{~N}$ & $\mathrm{~N}$ \\
\hline Ilha Bela & $\mathrm{N}$ & $\mathrm{N}$ & $\mathrm{P}$ & $\mathrm{S}$ & $\mathrm{S}$ & $\mathrm{S}$ & $\mathrm{S}$ & $\mathrm{P}$ & $\mathrm{N}$ & $\mathrm{N}$ & $\mathrm{N}$ & $\mathrm{N}$ \\
\hline Indaiatuba & $\mathrm{P}$ & $\mathrm{S}$ & $\mathrm{S}$ & NR & NR & NR & $\mathrm{S}$ & $\mathrm{S}$ & $\mathrm{S}$ & $\mathrm{S}$ & $\mathrm{P}$ & $\mathrm{P}$ \\
\hline Indiana & $\mathrm{P}$ & $\mathrm{S}$ & $\mathrm{S}$ & $\mathrm{S}$ & $\mathrm{S}$ & $\mathrm{S}$ & $\mathrm{S}$ & $\mathrm{S}$ & $\mathrm{P}$ & $\mathrm{P}$ & $\mathrm{P}$ & $\mathrm{P}$ \\
\hline Indiapora & $\mathrm{N}$ & $\mathrm{P}$ & $\mathrm{P}$ & $\mathrm{S}$ & $\mathrm{S}$ & $\mathrm{S}$ & $\mathrm{P}$ & $\mathrm{N}$ & $\mathrm{N}$ & $\mathrm{N}$ & $\mathrm{N}$ & $\mathrm{N}$ \\
\hline Inúbia Paulista & $\mathrm{N}$ & $\mathrm{P}$ & $\mathrm{S}$ & $\mathrm{S}$ & $\mathrm{S}$ & $\mathrm{S}$ & $\mathrm{S}$ & $\mathrm{P}$ & $\mathrm{P}$ & $\mathrm{N}$ & $\mathrm{N}$ & $\mathrm{N}$ \\
\hline Ipaucu & $\mathrm{P}$ & $\mathrm{S}$ & $\mathrm{S}$ & NR & NR & NR & $\mathrm{S}$ & $\mathrm{S}$ & $\mathrm{S}$ & $\mathrm{S}$ & $\mathrm{P}$ & $\mathrm{P}$ \\
\hline Iperó & $\mathrm{P}$ & $\mathrm{S}$ & $\mathrm{S}$ & NR & NR & NR & $\mathrm{S}$ & $\mathrm{S}$ & $\mathrm{S}$ & $\mathrm{S}$ & $\mathrm{P}$ & $\mathrm{P}$ \\
\hline Ipeúna & $\mathrm{S}$ & $\mathrm{S}$ & $\mathrm{S}$ & NR & NR & NR & $\mathrm{S}$ & $\mathrm{S}$ & $\mathrm{S}$ & $\mathrm{S}$ & $\mathrm{P}$ & $\mathrm{P}$ \\
\hline Ipiguá & $\mathrm{P}$ & $\mathrm{P}$ & $\mathrm{S}$ & $\mathrm{S}$ & $\mathrm{S}$ & $\mathrm{S}$ & $\mathrm{S}$ & $\mathrm{P}$ & $\mathrm{P}$ & $\mathrm{P}$ & $\mathrm{N}$ & $\mathrm{N}$ \\
\hline Iporanga & $\mathrm{S}$ & NR & NR & NR & NR & NR & NR & NR & NR & $\mathrm{S}$ & $\mathrm{S}$ & $\mathrm{S}$ \\
\hline Ipuã & $\mathrm{P}$ & $\mathrm{P}$ & $\mathrm{S}$ & $\mathrm{S}$ & $\mathrm{S}$ & $\mathrm{S}$ & $\mathrm{S}$ & $\mathrm{P}$ & $\mathrm{P}$ & $\mathrm{P}$ & $\mathrm{N}$ & $\mathrm{N}$ \\
\hline Iracemápolis & $\mathrm{S}$ & $\mathrm{S}$ & NR & NR & NR & NR & $\mathrm{S}$ & $\mathrm{S}$ & $\mathrm{S}$ & $\mathrm{S}$ & $\mathrm{S}$ & $\mathrm{S}$ \\
\hline Irapuã & $\mathrm{N}$ & $\mathrm{P}$ & $\mathrm{S}$ & $\mathrm{S}$ & $\mathrm{S}$ & $\mathrm{S}$ & $\mathrm{S}$ & $\mathrm{P}$ & $\mathrm{P}$ & $\mathrm{P}$ & $\mathrm{N}$ & $\mathrm{N}$ \\
\hline Irapuru & $\mathrm{P}$ & $\mathrm{P}$ & $\mathrm{S}$ & $\mathrm{S}$ & $\mathrm{S}$ & $\mathrm{S}$ & $\mathrm{S}$ & $\mathrm{P}$ & $\mathrm{P}$ & $\mathrm{P}$ & $\mathrm{N}$ & $\mathrm{N}$ \\
\hline Itabera & $\mathrm{S}$ & $\mathrm{S}$ & NR & NR & NR & NR & NR & $\mathrm{S}$ & $\mathrm{S}$ & $\mathrm{S}$ & $\mathrm{S}$ & $\mathrm{S}$ \\
\hline Itaí & $\mathrm{S}$ & $\mathrm{S}$ & NR & NR & NR & NR & NR & $\mathrm{S}$ & $\mathrm{S}$ & $\mathrm{S}$ & $\mathrm{S}$ & $\mathrm{P}$ \\
\hline Itajobi & $\mathrm{P}$ & $\mathrm{P}$ & $\mathrm{S}$ & $\mathrm{S}$ & $\mathrm{S}$ & $\mathrm{S}$ & $\mathrm{S}$ & $\mathrm{P}$ & $\mathrm{P}$ & $\mathrm{P}$ & $\mathrm{P}$ & $\mathrm{N}$ \\
\hline Itaju & $\mathrm{P}$ & $\mathrm{P}$ & $\mathrm{S}$ & $\mathrm{S}$ & $\mathrm{S}$ & $\mathrm{S}$ & $\mathrm{S}$ & $\mathrm{P}$ & $\mathrm{P}$ & $\mathrm{P}$ & $\mathrm{N}$ & $\mathrm{N}$ \\
\hline Itanhaém & $\mathrm{N}$ & $\mathrm{N}$ & $\mathrm{P}$ & $\mathrm{S}$ & $\mathrm{S}$ & $\mathrm{S}$ & $\mathrm{S}$ & $\mathrm{P}$ & $\mathrm{N}$ & $\mathrm{N}$ & $\mathrm{N}$ & $\mathrm{N}$ \\
\hline Itaoca & $\mathrm{S}$ & NR & NR & NR & NR & NR & NR & NR & $\mathrm{S}$ & $\mathrm{S}$ & $\mathrm{S}$ & $\mathrm{S}$ \\
\hline
\end{tabular}


Tabela 10. Genótipos de milho mais recomendados para cada início de ciclo nos municípios do Estado de São Paulo, durante os doze meses do ano. N: normal; P: precoce; S: superprecoce; NR: não recomendável

\begin{tabular}{|c|c|c|c|c|c|c|c|c|c|c|c|c|}
\hline \multirow{2}{*}{ Município } & \multicolumn{12}{|c|}{ Meses } \\
\hline & Jan & $\mathrm{Fev}$ & Mar & Abr & Mai & Jun & Jul & Ago & Set & Out & Nov & Dez \\
\hline Itapecirica da Serra & $\mathrm{S}$ & NR & NR & NR & NR & NR & $\mathrm{NR}$ & NR & $\mathrm{S}$ & $\mathrm{S}$ & $\mathrm{S}$ & $\mathrm{S}$ \\
\hline Itapetininga & $\mathrm{S}$ & $\mathrm{S}$ & NR & NR & NR & NR & NR & $\mathrm{S}$ & $\mathrm{S}$ & $\mathrm{S}$ & $\mathrm{S}$ & $\mathrm{S}$ \\
\hline Itapeva & $\mathrm{S}$ & $\mathrm{S}$ & NR & NR & NR & NR & NR & $\mathrm{S}$ & $\mathrm{S}$ & $\mathrm{S}$ & $\mathrm{S}$ & $\mathrm{S}$ \\
\hline Itapevi & $\mathrm{P}$ & $\mathrm{S}$ & $\mathrm{S}$ & NR & NR & NR & $\mathrm{S}$ & $\mathrm{S}$ & $\mathrm{S}$ & $\mathrm{S}$ & $\mathrm{P}$ & $\mathrm{P}$ \\
\hline Itapira & $\mathrm{S}$ & $\mathrm{S}$ & $\mathrm{S}$ & NR & NR & NR & $\mathrm{S}$ & $\mathrm{S}$ & $\mathrm{S}$ & $\mathrm{S}$ & $\mathrm{P}$ & $\mathrm{P}$ \\
\hline Itapirapuã Paulista & $\mathrm{P}$ & $\mathrm{S}$ & NR & NR & NR & NR & $\mathrm{S}$ & $\mathrm{S}$ & $\mathrm{S}$ & $\mathrm{S}$ & $\mathrm{P}$ & $\mathrm{P}$ \\
\hline Itápolis & $\mathrm{P}$ & $\mathrm{S}$ & $\mathrm{S}$ & $\mathrm{S}$ & $\mathrm{S}$ & $\mathrm{S}$ & $\mathrm{S}$ & $\mathrm{S}$ & $\mathrm{P}$ & $\mathrm{P}$ & $\mathrm{P}$ & $\mathrm{P}$ \\
\hline Itaporanga & $\mathrm{P}$ & $\mathrm{S}$ & $\mathrm{S}$ & NR & NR & NR & $\mathrm{S}$ & $\mathrm{S}$ & $\mathrm{S}$ & $\mathrm{S}$ & $\mathrm{P}$ & $\mathrm{P}$ \\
\hline Itapuí & $\mathrm{P}$ & $\mathrm{S}$ & $\mathrm{S}$ & $\mathrm{S}$ & $\mathrm{S}$ & $\mathrm{S}$ & $\mathrm{S}$ & $\mathrm{S}$ & $\mathrm{P}$ & $\mathrm{P}$ & $\mathrm{P}$ & $\mathrm{P}$ \\
\hline Itapura & $\mathrm{N}$ & $\mathrm{N}$ & $\mathrm{P}$ & $\mathrm{S}$ & $\mathrm{S}$ & $\mathrm{S}$ & $\mathrm{P}$ & $\mathrm{N}$ & $\mathrm{N}$ & $\mathrm{N}$ & $\mathrm{N}$ & $\mathrm{N}$ \\
\hline Itaquaquecetuba & $\mathrm{S}$ & $\mathrm{S}$ & NR & NR & NR & NR & NR & $\mathrm{S}$ & $\mathrm{S}$ & $\mathrm{S}$ & $\mathrm{S}$ & $\mathrm{S}$ \\
\hline Itararé & $\mathrm{S}$ & $\mathrm{S}$ & NR & NR & NR & NR & NR & $\mathrm{S}$ & $\mathrm{S}$ & $\mathrm{S}$ & $\mathrm{S}$ & $\mathrm{S}$ \\
\hline Itariri & $\mathrm{N}$ & $\mathrm{N}$ & $\mathrm{P}$ & $\mathrm{S}$ & $\mathrm{S}$ & $\mathrm{S}$ & $\mathrm{S}$ & $\mathrm{P}$ & $\mathrm{P}$ & $\mathrm{N}$ & $\mathrm{N}$ & $\mathrm{N}$ \\
\hline Itatiba & $\mathrm{S}$ & $\mathrm{S}$ & NR & NR & NR & NR & NR & $\mathrm{S}$ & $\mathrm{S}$ & $\mathrm{S}$ & $\mathrm{S}$ & $\mathrm{S}$ \\
\hline Itatinga & $\mathrm{S}$ & NR & NR & NR & NR & NR & NR & $\mathrm{S}$ & $\mathrm{S}$ & $\mathrm{S}$ & $\mathrm{S}$ & $\mathrm{S}$ \\
\hline Itirapina & $\mathrm{S}$ & $\mathrm{S}$ & NR & NR & NR & NR & $\mathrm{S}$ & $\mathrm{S}$ & $\mathrm{S}$ & $\mathrm{S}$ & $\mathrm{S}$ & $\mathrm{S}$ \\
\hline Itirapuã & $\mathrm{S}$ & $\mathrm{S}$ & NR & NR & NR & $\mathrm{S}$ & $\mathrm{S}$ & $\mathrm{S}$ & $\mathrm{S}$ & $\mathrm{S}$ & $\mathrm{S}$ & $\mathrm{S}$ \\
\hline Itobi & $\mathrm{S}$ & $\mathrm{S}$ & $\mathrm{S}$ & NR & NR & $\mathrm{S}$ & $\mathrm{S}$ & $\mathrm{S}$ & $\mathrm{S}$ & $\mathrm{S}$ & $\mathrm{P}$ & $\mathrm{P}$ \\
\hline Itu & $\mathrm{P}$ & $\mathrm{S}$ & $\mathrm{S}$ & NR & NR & NR & $\mathrm{S}$ & $\mathrm{S}$ & $\mathrm{S}$ & $\mathrm{S}$ & $\mathrm{P}$ & $\mathrm{P}$ \\
\hline Itupeva & $\mathrm{P}$ & $\mathrm{S}$ & $\mathrm{S}$ & NR & NR & NR & $\mathrm{S}$ & $\mathrm{S}$ & $\mathrm{S}$ & $\mathrm{P}$ & $\mathrm{P}$ & $\mathrm{P}$ \\
\hline Ituverava & $\mathrm{P}$ & $\mathrm{P}$ & $\mathrm{S}$ & $\mathrm{S}$ & $\mathrm{S}$ & $\mathrm{S}$ & $\mathrm{S}$ & $\mathrm{P}$ & $\mathrm{P}$ & $\mathrm{P}$ & $\mathrm{P}$ & $\mathrm{P}$ \\
\hline Jaborandi & $\mathrm{P}$ & $\mathrm{P}$ & $\mathrm{S}$ & $\mathrm{S}$ & $\mathrm{S}$ & $\mathrm{S}$ & $\mathrm{S}$ & $\mathrm{P}$ & $\mathrm{P}$ & $\mathrm{P}$ & $\mathrm{N}$ & $\mathrm{N}$ \\
\hline Jaboticabal & $P$ & $\mathrm{~S}$ & $\mathrm{~S}$ & $\mathrm{~S}$ & $\mathrm{~S}$ & $\mathrm{~S}$ & $\mathrm{~S}$ & $\mathrm{~S}$ & $\mathrm{P}$ & $\mathrm{P}$ & $\mathrm{P}$ & $\mathrm{P}$ \\
\hline Jacareí & $P$ & $\mathrm{~S}$ & $\mathrm{~S}$ & NR & NR & NR & $\mathrm{S}$ & $\mathrm{S}$ & $\mathrm{S}$ & $\mathrm{S}$ & $\mathrm{P}$ & $\mathrm{P}$ \\
\hline Jaci & $\mathrm{P}$ & $\mathrm{P}$ & $\mathrm{S}$ & $\mathrm{S}$ & $\mathrm{S}$ & $\mathrm{S}$ & $\mathrm{S}$ & $\mathrm{P}$ & $\mathrm{P}$ & $\mathrm{P}$ & $\mathrm{N}$ & $\mathrm{N}$ \\
\hline Jacupiranga & $\mathrm{N}$ & $\mathrm{P}$ & $\mathrm{S}$ & $\mathrm{S}$ & $\mathrm{S}$ & $\mathrm{S}$ & $\mathrm{S}$ & $\mathrm{S}$ & $\mathrm{P}$ & $\mathrm{P}$ & $\mathrm{N}$ & $\mathrm{N}$ \\
\hline Jaguariúna & $\mathrm{P}$ & $\mathrm{S}$ & $\mathrm{S}$ & NR & NR & NR & $\mathrm{S}$ & $\mathrm{S}$ & $\mathrm{S}$ & S & $\mathrm{P}$ & $\mathrm{P}$ \\
\hline Jales & $\mathrm{N}$ & $\mathrm{P}$ & $\mathrm{P}$ & $\mathrm{S}$ & $\mathrm{S}$ & $\mathrm{S}$ & $\mathrm{P}$ & $\mathrm{N}$ & $\mathrm{N}$ & $\mathrm{N}$ & $\mathrm{N}$ & $\mathrm{N}$ \\
\hline Jambeiro & $\mathrm{S}$ & $\mathrm{S}$ & NR & NR & NR & NR & NR & $\mathrm{S}$ & $\mathrm{S}$ & $\mathrm{S}$ & $\mathrm{S}$ & $\mathrm{S}$ \\
\hline Jandira & $\mathrm{S}$ & $\mathrm{S}$ & NR & NR & NR & NR & $\mathrm{S}$ & $\mathrm{S}$ & $\mathrm{S}$ & $\mathrm{S}$ & $\mathrm{P}$ & $\mathrm{P}$ \\
\hline Jardinópolis & $\mathrm{P}$ & S & $\mathrm{S}$ & $\mathrm{S}$ & $\mathrm{S}$ & $\mathrm{S}$ & $\mathrm{S}$ & $\mathrm{S}$ & $\mathrm{P}$ & $\mathrm{P}$ & $\mathrm{P}$ & $\mathrm{P}$ \\
\hline Jarinu & $\mathrm{S}$ & $\mathrm{S}$ & NR & NR & NR & NR & $\mathrm{S}$ & $\mathrm{S}$ & $\mathrm{S}$ & $\mathrm{S}$ & $\mathrm{S}$ & $\mathrm{P}$ \\
\hline Jaú & $\mathrm{P}$ & $\mathrm{S}$ & $\mathrm{S}$ & $\mathrm{S}$ & NR & $\mathrm{S}$ & $\mathrm{S}$ & $\mathrm{S}$ & $\mathrm{S}$ & $\mathrm{P}$ & $\mathrm{P}$ & $\mathrm{P}$ \\
\hline Jeriquara & $\mathrm{S}$ & $\mathrm{S}$ & NR & NR & NR & $\mathrm{S}$ & $\mathrm{S}$ & $\mathrm{S}$ & $\mathrm{S}$ & $\mathrm{S}$ & $\mathrm{S}$ & $\mathrm{S}$ \\
\hline Joanópolis & $\mathrm{S}$ & NR & NR & NR & NR & NR & NR & NR & $\mathrm{S}$ & $\mathrm{S}$ & $\mathrm{S}$ & $\mathrm{S}$ \\
\hline João Ramalho & $\mathrm{P}$ & $\mathrm{P}$ & $\mathrm{S}$ & $\mathrm{S}$ & $\mathrm{S}$ & $\mathrm{S}$ & $\mathrm{S}$ & $\mathrm{S}$ & $\mathrm{P}$ & $\mathrm{P}$ & $\mathrm{P}$ & $\mathrm{P}$ \\
\hline José Bonifácio & $\mathrm{N}$ & $\mathrm{P}$ & $\mathrm{S}$ & $\mathrm{S}$ & $\mathrm{S}$ & $\mathrm{S}$ & $\mathrm{S}$ & $\mathrm{P}$ & $\mathrm{P}$ & $\mathrm{N}$ & $\mathrm{N}$ & $\mathrm{N}$ \\
\hline Júlio Mesquita & $\mathrm{P}$ & $\mathrm{S}$ & $\mathrm{S}$ & $\mathrm{S}$ & $\mathrm{S}$ & $\mathrm{S}$ & $\mathrm{S}$ & $\mathrm{S}$ & $\mathrm{P}$ & $\mathrm{P}$ & $\mathrm{P}$ & $\mathrm{P}$ \\
\hline Jumirim & $\mathrm{P}$ & $\mathrm{S}$ & $\mathrm{S}$ & NR & NR & NR & $\mathrm{S}$ & $\mathrm{S}$ & $\mathrm{S}$ & S & $\mathrm{P}$ & $\mathrm{P}$ \\
\hline Jundiaí & $\mathrm{S}$ & $\mathrm{S}$ & NR & NR & NR & NR & NR & $\mathrm{S}$ & $\mathrm{S}$ & $\mathrm{S}$ & $\mathrm{S}$ & $\mathrm{P}$ \\
\hline Junqueirópolis & $\mathrm{N}$ & $\mathrm{P}$ & $\mathrm{P}$ & $\mathrm{S}$ & $\mathrm{S}$ & $\mathrm{S}$ & $\mathrm{S}$ & $\mathrm{P}$ & $\mathrm{N}$ & $\mathrm{N}$ & $\mathrm{N}$ & $\mathrm{N}$ \\
\hline Juquiá & $\mathrm{N}$ & $\mathrm{N}$ & $\mathrm{P}$ & $\mathrm{S}$ & $\mathrm{S}$ & $\mathrm{S}$ & $\mathrm{S}$ & $\mathrm{P}$ & $\mathrm{P}$ & $\mathrm{N}$ & $\mathrm{N}$ & $\mathrm{N}$ \\
\hline Juquitiba & $\mathrm{S}$ & NR & NR & NR & NR & NR & NR & NR & $\mathrm{S}$ & $\mathrm{S}$ & $\mathrm{S}$ & $\mathrm{S}$ \\
\hline
\end{tabular}


Tabela 10. Genótipos de milho mais recomendados para cada início de ciclo nos municípios do Estado de São Paulo, durante os doze meses do ano. N: normal; P: precoce; S: superprecoce; NR: não recomendável

\begin{tabular}{|c|c|c|c|c|c|c|c|c|c|c|c|c|}
\hline \multirow{2}{*}{ Município } & \multicolumn{12}{|c|}{ Meses } \\
\hline & Jan & $\mathrm{Fev}$ & Mar & Abr & Mai & Jun & Jul & Ago & Set & Out & Nov & Dez \\
\hline Lagoinha & $\mathrm{S}$ & NR & NR & NR & NR & NR & NR & $\mathrm{S}$ & $\mathrm{S}$ & $\mathrm{S}$ & $\mathrm{S}$ & $\bar{S}$ \\
\hline Laranjal Paulista & $\mathrm{P}$ & $\mathrm{S}$ & $\mathrm{S}$ & NR & NR & $\mathrm{S}$ & $\mathrm{S}$ & $\mathrm{S}$ & $\mathrm{S}$ & $\mathrm{P}$ & $\mathrm{P}$ & $\mathrm{P}$ \\
\hline Lavínia & $\mathrm{N}$ & $\mathrm{P}$ & $\mathrm{S}$ & $\mathrm{S}$ & $\mathrm{S}$ & $\mathrm{S}$ & $\mathrm{S}$ & $\mathrm{P}$ & $\mathrm{N}$ & $\mathrm{N}$ & $\mathrm{N}$ & $\mathrm{N}$ \\
\hline Lavrinhas & $\mathrm{S}$ & $\mathrm{S}$ & NR & NR & NR & NR & $\mathrm{S}$ & $\mathrm{S}$ & $\mathrm{S}$ & $\mathrm{S}$ & $\mathrm{S}$ & $\mathrm{P}$ \\
\hline Leme & $\mathrm{P}$ & $\mathrm{S}$ & $\mathrm{S}$ & NR & NR & $\mathrm{S}$ & $\mathrm{S}$ & $\mathrm{S}$ & $\mathrm{S}$ & $\mathrm{S}$ & $\mathrm{P}$ & $\mathrm{P}$ \\
\hline Lençóis Paulista & $\mathrm{P}$ & $\mathrm{S}$ & $\mathrm{S}$ & NR & NR & $\mathrm{S}$ & $\mathrm{S}$ & $\mathrm{S}$ & $\mathrm{S}$ & $\mathrm{P}$ & $\mathrm{P}$ & $\mathrm{P}$ \\
\hline Limeira & $\mathrm{S}$ & $\mathrm{S}$ & NR & NR & NR & NR & $\mathrm{S}$ & $\mathrm{S}$ & $\mathrm{S}$ & $\mathrm{S}$ & $\mathrm{S}$ & $\mathrm{S}$ \\
\hline Lindóia & $\mathrm{S}$ & $\mathrm{S}$ & $\mathrm{S}$ & NR & NR & NR & $\mathrm{S}$ & $\mathrm{S}$ & $\mathrm{S}$ & $\mathrm{S}$ & $\mathrm{S}$ & $\mathrm{P}$ \\
\hline Lins & $\mathrm{N}$ & $\mathrm{P}$ & $\mathrm{S}$ & $\mathrm{S}$ & $\mathrm{S}$ & $\mathrm{S}$ & $\mathrm{S}$ & $\mathrm{P}$ & $\mathrm{P}$ & $\mathrm{P}$ & $\mathrm{N}$ & $\mathrm{N}$ \\
\hline Lorena & $\mathrm{P}$ & $\mathrm{S}$ & $\mathrm{S}$ & NR & NR & $\mathrm{S}$ & $\mathrm{S}$ & $\mathrm{S}$ & $\mathrm{S}$ & $\mathrm{P}$ & $\mathrm{P}$ & $\mathrm{P}$ \\
\hline Lourdes & $\mathrm{N}$ & $\mathrm{P}$ & $\mathrm{S}$ & $\mathrm{S}$ & $\mathrm{S}$ & $\mathrm{S}$ & $\mathrm{S}$ & $\mathrm{P}$ & $\mathrm{N}$ & $\mathrm{N}$ & $\mathrm{N}$ & $\mathrm{N}$ \\
\hline Louveira & $\mathrm{P}$ & $\mathrm{S}$ & $\mathrm{S}$ & NR & NR & NR & $\mathrm{S}$ & $\mathrm{S}$ & $\mathrm{S}$ & $\mathrm{P}$ & $\mathrm{P}$ & $\mathrm{P}$ \\
\hline Lucélia & $\mathrm{P}$ & $\mathrm{P}$ & $\mathrm{S}$ & $\mathrm{S}$ & $\mathrm{S}$ & $\mathrm{S}$ & $\mathrm{S}$ & $\mathrm{S}$ & $\mathrm{P}$ & $\mathrm{P}$ & $\mathrm{P}$ & $\mathrm{N}$ \\
\hline Lucianópolis & $\mathrm{P}$ & $\mathrm{S}$ & $\mathrm{S}$ & NR & NR & $\mathrm{S}$ & $\mathrm{S}$ & $\mathrm{S}$ & $\mathrm{S}$ & $\mathrm{P}$ & $\mathrm{P}$ & $\mathrm{P}$ \\
\hline Luis Antônio & $\mathrm{P}$ & $\mathrm{S}$ & $\mathrm{S}$ & $\mathrm{S}$ & $\mathrm{S}$ & $\mathrm{S}$ & $\mathrm{S}$ & $\mathrm{S}$ & $\mathrm{S}$ & $\mathrm{P}$ & $\mathrm{P}$ & $\mathrm{P}$ \\
\hline Luiziânia & $\mathrm{P}$ & $\mathrm{P}$ & $\mathrm{S}$ & $\mathrm{S}$ & $\mathrm{S}$ & $\mathrm{S}$ & $\mathrm{S}$ & $\mathrm{P}$ & $\mathrm{P}$ & $\mathrm{P}$ & $\mathrm{N}$ & $\mathrm{N}$ \\
\hline Lupércio & $\mathrm{P}$ & $\mathrm{S}$ & $\mathrm{S}$ & NR & NR & $\mathrm{S}$ & $\mathrm{S}$ & $\mathrm{S}$ & $\mathrm{S}$ & $\mathrm{P}$ & $\mathrm{P}$ & $\mathrm{P}$ \\
\hline Lutécia & $\mathrm{P}$ & $\mathrm{S}$ & $\mathrm{S}$ & $\mathrm{S}$ & $\mathrm{S}$ & $\mathrm{S}$ & $\mathrm{S}$ & $\mathrm{S}$ & $\mathrm{P}$ & $\mathrm{P}$ & $\mathrm{P}$ & $\mathrm{P}$ \\
\hline Macatuba & $\mathrm{P}$ & $\mathrm{S}$ & $\mathrm{S}$ & $\mathrm{S}$ & $\mathrm{S}$ & $\mathrm{S}$ & $\mathrm{S}$ & $\mathrm{S}$ & $\mathrm{P}$ & $\mathrm{P}$ & $\mathrm{P}$ & $\mathrm{P}$ \\
\hline Macaubal & $\mathrm{N}$ & $\mathrm{P}$ & $\mathrm{S}$ & $\mathrm{S}$ & $\mathrm{S}$ & $\mathrm{S}$ & $\mathrm{S}$ & $\mathrm{P}$ & $\mathrm{N}$ & $\mathrm{N}$ & $\mathrm{N}$ & $\mathrm{N}$ \\
\hline Macedônia & $\mathrm{P}$ & $\mathrm{P}$ & $\mathrm{S}$ & $\mathrm{S}$ & $\mathrm{S}$ & $\mathrm{S}$ & $\mathrm{S}$ & $\mathrm{P}$ & $\mathrm{P}$ & $\mathrm{P}$ & $\mathrm{N}$ & $\mathrm{N}$ \\
\hline Magda & $\mathrm{N}$ & $\mathrm{P}$ & $\mathrm{S}$ & $\mathrm{S}$ & $\mathrm{S}$ & $\mathrm{S}$ & $\mathrm{P}$ & $\mathrm{P}$ & $\mathrm{N}$ & $\mathrm{N}$ & $\mathrm{N}$ & $\mathrm{N}$ \\
\hline Mairinque & $\mathrm{P}$ & $\mathrm{S}$ & $\mathrm{S}$ & NR & NR & NR & $\mathrm{S}$ & $\mathrm{S}$ & $\mathrm{S}$ & $\mathrm{S}$ & $\mathrm{P}$ & $\mathrm{P}$ \\
\hline Mairipora & $\mathrm{S}$ & $\mathrm{S}$ & NR & NR & NR & NR & NR & $\mathrm{S}$ & $\mathrm{S}$ & $\mathrm{S}$ & $\mathrm{S}$ & $\mathrm{S}$ \\
\hline Manduri & $\mathrm{S}$ & $\mathrm{S}$ & NR & NR & NR & NR & NR & $\mathrm{S}$ & $\mathrm{S}$ & $\mathrm{S}$ & $\mathrm{P}$ & $\mathrm{P}$ \\
\hline Marabá Paulista & $\mathrm{N}$ & $\mathrm{P}$ & $\mathrm{S}$ & $\mathrm{S}$ & $\mathrm{S}$ & $\mathrm{S}$ & $\mathrm{S}$ & $\mathrm{P}$ & $\mathrm{P}$ & $\mathrm{N}$ & $\mathrm{N}$ & $\mathrm{N}$ \\
\hline Maracaí & $\mathrm{P}$ & $\mathrm{P}$ & $\mathrm{S}$ & $\mathrm{S}$ & $\mathrm{S}$ & $\mathrm{S}$ & $\mathrm{S}$ & $\mathrm{S}$ & $\mathrm{P}$ & $\mathrm{P}$ & $\mathrm{P}$ & $\mathrm{N}$ \\
\hline Marapoama & $\mathrm{P}$ & $\mathrm{P}$ & $\mathrm{S}$ & $\mathrm{S}$ & $\mathrm{S}$ & $\mathrm{S}$ & $\mathrm{S}$ & $\mathrm{P}$ & $\mathrm{P}$ & $\mathrm{P}$ & $\mathrm{P}$ & $\mathrm{P}$ \\
\hline Mariápolis & $\mathrm{N}$ & $\mathrm{P}$ & $\mathrm{S}$ & $\mathrm{S}$ & $\mathrm{S}$ & $\mathrm{S}$ & $\mathrm{S}$ & $\mathrm{P}$ & $\mathrm{N}$ & $\mathrm{N}$ & $\mathrm{N}$ & $\mathrm{N}$ \\
\hline Marília & $\mathrm{S}$ & $\mathrm{S}$ & $\mathrm{S}$ & NR & NR & NR & $\mathrm{S}$ & $\mathrm{S}$ & $\mathrm{S}$ & $\mathrm{S}$ & $\mathrm{P}$ & $\mathrm{P}$ \\
\hline Marinópolis & $\mathrm{N}$ & $\mathrm{P}$ & $\mathrm{P}$ & $\mathrm{S}$ & $\mathrm{S}$ & $\mathrm{S}$ & $\mathrm{P}$ & $\mathrm{P}$ & $\mathrm{N}$ & $\mathrm{N}$ & $\mathrm{N}$ & $\mathrm{N}$ \\
\hline Martinópolis & $\mathrm{P}$ & $\mathrm{S}$ & $\mathrm{S}$ & $\mathrm{S}$ & $\mathrm{S}$ & $\mathrm{S}$ & $\mathrm{S}$ & $\mathrm{S}$ & $\mathrm{P}$ & $\mathrm{P}$ & $\mathrm{P}$ & $\mathrm{P}$ \\
\hline Matão & $\mathrm{P}$ & $\mathrm{S}$ & $\mathrm{S}$ & $\mathrm{S}$ & $\mathrm{S}$ & $\mathrm{S}$ & $\mathrm{S}$ & $\mathrm{S}$ & $\mathrm{P}$ & $\mathrm{P}$ & $\mathrm{P}$ & $\mathrm{P}$ \\
\hline Mauá & $\mathrm{S}$ & NR & NR & NR & NR & NR & NR & NR & $\mathrm{S}$ & $\mathrm{S}$ & $\mathrm{S}$ & $\mathrm{S}$ \\
\hline Mendonça & $\mathrm{P}$ & $\mathrm{S}$ & $\mathrm{S}$ & $\mathrm{S}$ & $\mathrm{S}$ & $\mathrm{S}$ & $\mathrm{S}$ & $\mathrm{S}$ & $\mathrm{P}$ & $\mathrm{P}$ & $\mathrm{P}$ & $\mathrm{P}$ \\
\hline Meridiano & $\mathrm{N}$ & $\mathrm{P}$ & $\mathrm{S}$ & $\mathrm{S}$ & $\mathrm{S}$ & $\mathrm{S}$ & $\mathrm{P}$ & $\mathrm{P}$ & $\mathrm{N}$ & $\mathrm{N}$ & $\mathrm{N}$ & $\mathrm{N}$ \\
\hline Mesópolis & $\mathrm{N}$ & $\mathrm{N}$ & $\mathrm{P}$ & $\mathrm{S}$ & $\mathrm{S}$ & $\mathrm{S}$ & $\mathrm{P}$ & $\mathrm{N}$ & $\mathrm{N}$ & $\mathrm{N}$ & $\mathrm{N}$ & $\mathrm{N}$ \\
\hline Miguelópolis & $\mathrm{P}$ & $\mathrm{P}$ & $\mathrm{S}$ & $\mathrm{S}$ & $\mathrm{S}$ & $\mathrm{S}$ & $\mathrm{S}$ & $\mathrm{P}$ & $\mathrm{P}$ & $\mathrm{P}$ & $\mathrm{N}$ & $\mathrm{N}$ \\
\hline Mineiros do Tietê & $\mathrm{P}$ & $\mathrm{S}$ & $\mathrm{S}$ & $\mathrm{S}$ & $\mathrm{S}$ & $\mathrm{S}$ & $\mathrm{S}$ & $\mathrm{S}$ & $\mathrm{P}$ & $\mathrm{P}$ & $\mathrm{P}$ & $\mathrm{P}$ \\
\hline Mira Estrela & $\mathrm{N}$ & $\mathrm{P}$ & $\mathrm{P}$ & $\mathrm{S}$ & $\mathrm{S}$ & $\mathrm{S}$ & $\mathrm{P}$ & $\mathrm{N}$ & $\mathrm{N}$ & $\mathrm{N}$ & $\mathrm{N}$ & $\mathrm{N}$ \\
\hline Miracatu & $\mathrm{N}$ & $\mathrm{N}$ & $\mathrm{P}$ & $\mathrm{S}$ & $\mathrm{S}$ & $\mathrm{S}$ & $\mathrm{S}$ & $\mathrm{P}$ & $\mathrm{P}$ & $\mathrm{N}$ & $\mathrm{N}$ & $\mathrm{N}$ \\
\hline Mirandópolis & $\mathrm{N}$ & $\mathrm{P}$ & $\mathrm{S}$ & $\mathrm{S}$ & $\mathrm{S}$ & $\mathrm{S}$ & $\mathrm{S}$ & $\mathrm{P}$ & $\mathrm{P}$ & $\mathrm{P}$ & $\mathrm{N}$ & $\mathrm{N}$ \\
\hline M. do Paranapanema & $\mathrm{N}$ & $\mathrm{P}$ & $\mathrm{S}$ & $\mathrm{S}$ & $\mathrm{S}$ & $\mathrm{S}$ & $\mathrm{S}$ & $\mathrm{P}$ & $\mathrm{P}$ & $\mathrm{N}$ & $\mathrm{N}$ & $\mathrm{N}$ \\
\hline
\end{tabular}


Tabela 10. Genótipos de milho mais recomendados para cada início de ciclo nos municípios do Estado de São Paulo, durante os doze meses do ano. N: normal; P: precoce; S: superprecoce; NR: não recomendável

\begin{tabular}{|c|c|c|c|c|c|c|c|c|c|c|c|c|}
\hline \multirow{2}{*}{ Município } & \multicolumn{12}{|c|}{ Meses } \\
\hline & Jan & $\mathrm{Fev}$ & Mar & Abr & Mai & Jun & Jul & Ago & Set & Out & Nov & Dez \\
\hline Mirassol & $\mathrm{P}$ & $\mathrm{S}$ & $\mathrm{S}$ & $\mathrm{S}$ & $\mathrm{S}$ & $\mathrm{S}$ & $\mathrm{S}$ & $\mathrm{S}$ & $\mathrm{P}$ & $\mathrm{P}$ & $\mathrm{P}$ & $\mathrm{P}$ \\
\hline Mirassolândia & $\mathrm{P}$ & $P$ & $\mathrm{~S}$ & $\mathrm{~S}$ & $\mathrm{~S}$ & $\mathrm{~S}$ & $\mathrm{~S}$ & $\mathrm{P}$ & $\mathrm{P}$ & $\mathrm{P}$ & $\mathrm{P}$ & $\mathrm{N}$ \\
\hline Mococa & $\mathrm{P}$ & $\mathrm{S}$ & $\mathrm{S}$ & $\mathrm{S}$ & $\mathrm{S}$ & $\mathrm{S}$ & $\mathrm{S}$ & $\mathrm{S}$ & $\mathrm{S}$ & $\mathrm{P}$ & $\mathrm{P}$ & $\mathrm{P}$ \\
\hline Mogii-Guaçu & $\mathrm{S}$ & $\mathrm{S}$ & $\mathrm{S}$ & NR & NR & $\mathrm{S}$ & $\mathrm{S}$ & $\mathrm{S}$ & $\mathrm{S}$ & $\mathrm{S}$ & $\mathrm{P}$ & $\mathrm{P}$ \\
\hline Mogi das Cruzes & $\mathrm{S}$ & $\mathrm{S}$ & NR & NR & NR & NR & NR & $\mathrm{S}$ & $\mathrm{S}$ & $\mathrm{S}$ & $\mathrm{S}$ & $\mathrm{S}$ \\
\hline Mogi Mirim & $\mathrm{P}$ & $\mathrm{S}$ & $\mathrm{S}$ & NR & NR & NR & $\mathrm{S}$ & $\mathrm{S}$ & $\mathrm{S}$ & $\mathrm{S}$ & $\mathrm{P}$ & $\mathrm{P}$ \\
\hline Mombuca & $\mathrm{S}$ & $\mathrm{S}$ & NR & NR & NR & NR & $\mathrm{S}$ & $\mathrm{S}$ & $\mathrm{S}$ & $\mathrm{S}$ & $\mathrm{P}$ & $\mathrm{P}$ \\
\hline Monções & $\mathrm{N}$ & $\mathrm{P}$ & $\mathrm{S}$ & $\mathrm{S}$ & $\mathrm{S}$ & $\mathrm{S}$ & $\mathrm{S}$ & $\mathrm{P}$ & $\mathrm{N}$ & $\mathrm{N}$ & $\mathrm{N}$ & $\mathrm{N}$ \\
\hline Monguágua & $\mathrm{N}$ & $\mathrm{N}$ & $\mathrm{P}$ & $\mathrm{S}$ & $\mathrm{S}$ & $\mathrm{S}$ & $\mathrm{S}$ & $\mathrm{P}$ & $\mathrm{N}$ & $\mathrm{N}$ & $\mathrm{N}$ & $\mathrm{N}$ \\
\hline Monte Alegre do Sul & $\mathrm{S}$ & $\mathrm{S}$ & NR & NR & NR & NR & $\mathrm{S}$ & $\mathrm{S}$ & $\mathrm{S}$ & $\mathrm{S}$ & $\mathrm{S}$ & $\mathrm{S}$ \\
\hline Monte Alto & $\mathrm{S}$ & $\mathrm{S}$ & $\mathrm{S}$ & NR & NR & $\mathrm{S}$ & $\mathrm{S}$ & $\mathrm{S}$ & $\mathrm{S}$ & $\mathrm{S}$ & $\mathrm{P}$ & $\mathrm{P}$ \\
\hline Monte Aprazível & $\mathrm{P}$ & $\mathrm{P}$ & $\mathrm{S}$ & $\mathrm{S}$ & $\mathrm{S}$ & $\mathrm{S}$ & $\mathrm{S}$ & $\mathrm{P}$ & $\mathrm{P}$ & $\mathrm{P}$ & $\mathrm{N}$ & $\mathrm{N}$ \\
\hline Monte Azul Paulista & $\mathrm{P}$ & $\mathrm{S}$ & $\mathrm{S}$ & $\mathrm{S}$ & $\mathrm{S}$ & $\mathrm{S}$ & $\mathrm{S}$ & $\mathrm{S}$ & $\mathrm{P}$ & $\mathrm{P}$ & $\mathrm{P}$ & $\mathrm{P}$ \\
\hline Monte Castelo & $\mathrm{N}$ & $\mathrm{P}$ & $\mathrm{S}$ & $\mathrm{S}$ & $\mathrm{S}$ & $\mathrm{S}$ & $\mathrm{S}$ & $\mathrm{P}$ & $\mathrm{N}$ & $\mathrm{N}$ & $\mathrm{N}$ & $\mathrm{N}$ \\
\hline Monte Mor & $\mathrm{P}$ & $\mathrm{S}$ & $\mathrm{S}$ & NR & NR & NR & $\mathrm{S}$ & $\mathrm{S}$ & $\mathrm{S}$ & $\mathrm{P}$ & $\mathrm{P}$ & $\mathrm{P}$ \\
\hline Monteiro Lobato & $\mathrm{S}$ & $\mathrm{S}$ & NR & NR & NR & NR & NR & $\mathrm{S}$ & $\mathrm{S}$ & $\mathrm{S}$ & $\mathrm{S}$ & $\mathrm{S}$ \\
\hline Morro Agudo & $\mathrm{P}$ & $\mathrm{P}$ & $\mathrm{S}$ & $\mathrm{S}$ & S & $\mathrm{S}$ & $\mathrm{S}$ & $\mathrm{P}$ & $\mathrm{P}$ & $\mathrm{P}$ & $\mathrm{P}$ & $\mathrm{P}$ \\
\hline Morungaba & $\mathrm{S}$ & NR & NR & NR & NR & NR & NR & $\mathrm{S}$ & $\mathrm{S}$ & $\mathrm{S}$ & $\mathrm{S}$ & $\mathrm{S}$ \\
\hline Motuca & $\mathrm{P}$ & $\mathrm{S}$ & $\mathrm{S}$ & $\mathrm{S}$ & $\mathrm{S}$ & $\mathrm{S}$ & $\mathrm{S}$ & $\mathrm{S}$ & $\mathrm{S}$ & $\mathrm{P}$ & $\mathrm{P}$ & $\mathrm{P}$ \\
\hline Murutinga do Sul & $\mathrm{N}$ & $\mathrm{P}$ & $\mathrm{P}$ & $\mathrm{S}$ & $\mathrm{S}$ & $\mathrm{S}$ & $\mathrm{S}$ & $\mathrm{P}$ & $\mathrm{N}$ & $\mathrm{N}$ & $\mathrm{N}$ & $\mathrm{N}$ \\
\hline Nantes & $\mathrm{P}$ & $\mathrm{P}$ & $\mathrm{S}$ & $\mathrm{S}$ & $\mathrm{S}$ & $\mathrm{S}$ & $\mathrm{S}$ & $\mathrm{S}$ & $\mathrm{P}$ & $\mathrm{P}$ & $\mathrm{N}$ & $\mathrm{N}$ \\
\hline Narandiba & $\mathrm{P}$ & $\mathrm{P}$ & $\mathrm{S}$ & $\mathrm{S}$ & $\mathrm{S}$ & $\mathrm{S}$ & $\mathrm{S}$ & $\mathrm{S}$ & $\mathrm{P}$ & $\mathrm{P}$ & $\mathrm{P}$ & $\mathrm{N}$ \\
\hline Natividade da Serra & $\mathrm{S}$ & $\mathrm{S}$ & NR & NR & NR & NR & NR & $\mathrm{S}$ & $\mathrm{S}$ & $\mathrm{S}$ & $\mathrm{S}$ & $\mathrm{S}$ \\
\hline Nazaré Paulista & $\mathrm{S}$ & NR & NR & NR & NR & NR & NR & NR & $\mathrm{S}$ & $\mathrm{S}$ & $\mathrm{S}$ & $\mathrm{S}$ \\
\hline Neves Paulista & $\mathrm{P}$ & $\mathrm{P}$ & $\mathrm{S}$ & $\mathrm{S}$ & $\mathrm{S}$ & $\mathrm{S}$ & $\mathrm{S}$ & $\mathrm{P}$ & $\mathrm{P}$ & $\mathrm{P}$ & $\mathrm{N}$ & $\mathrm{N}$ \\
\hline Nhandeara & $\mathrm{N}$ & $P$ & S & $\mathrm{S}$ & $\mathrm{S}$ & $\mathrm{S}$ & $\mathrm{S}$ & $\mathrm{P}$ & $\mathrm{N}$ & $\mathrm{N}$ & $\mathrm{N}$ & $\mathrm{N}$ \\
\hline Nipoa & $\mathrm{N}$ & $\mathrm{P}$ & $\mathrm{S}$ & $\mathrm{S}$ & $\mathrm{S}$ & $\mathrm{S}$ & $\mathrm{S}$ & $\mathrm{P}$ & $\mathrm{P}$ & $\mathrm{P}$ & $\mathrm{N}$ & $\mathrm{N}$ \\
\hline Nova Aliança & $\mathrm{P}$ & $\mathrm{P}$ & $\mathrm{S}$ & $\mathrm{S}$ & $\mathrm{S}$ & $\mathrm{S}$ & $\mathrm{S}$ & $\mathrm{P}$ & $\mathrm{P}$ & $\mathrm{P}$ & $\mathrm{N}$ & $\mathrm{N}$ \\
\hline Nova Campina & $\mathrm{S}$ & S & NR & NR & NR & NR & NR & $\mathrm{S}$ & $\mathrm{S}$ & $\mathrm{S}$ & $\mathrm{P}$ & $\mathrm{P}$ \\
\hline Nova Canaã Paulista & $\mathrm{N}$ & $\mathrm{P}$ & $\mathrm{P}$ & $\mathrm{S}$ & $\mathrm{S}$ & $\mathrm{S}$ & $\mathrm{P}$ & $\mathrm{P}$ & $\mathrm{N}$ & $\mathrm{N}$ & $\mathrm{N}$ & $\mathrm{N}$ \\
\hline Nova Castilho & $\mathrm{N}$ & $\mathrm{P}$ & $\mathrm{P}$ & $\mathrm{S}$ & $\mathrm{S}$ & $\mathrm{S}$ & $\mathrm{P}$ & $\mathrm{P}$ & $\mathrm{N}$ & $\mathrm{N}$ & $\mathrm{N}$ & $\mathrm{N}$ \\
\hline Nova Europa & $\mathrm{P}$ & $\mathrm{P}$ & $\mathrm{S}$ & $\mathrm{S}$ & $\mathrm{S}$ & $\mathrm{S}$ & $\mathrm{S}$ & $\mathrm{S}$ & $\mathrm{P}$ & $\mathrm{P}$ & $\mathrm{P}$ & $\mathrm{P}$ \\
\hline Nova Granada & $\mathrm{P}$ & $\mathrm{P}$ & $\mathrm{S}$ & $\mathrm{S}$ & $\mathrm{S}$ & $\mathrm{S}$ & $\mathrm{S}$ & $\mathrm{P}$ & $\mathrm{P}$ & $\mathrm{N}$ & $\mathrm{N}$ & $\mathrm{N}$ \\
\hline Nova Guataporanga & $\mathrm{N}$ & $\mathrm{P}$ & $\mathrm{P}$ & $\mathrm{S}$ & $\mathrm{S}$ & $\mathrm{S}$ & $\mathrm{S}$ & $\mathrm{P}$ & $\mathrm{N}$ & $\mathrm{N}$ & $\mathrm{N}$ & $\mathrm{N}$ \\
\hline Nova Independência & $\mathrm{N}$ & $\mathrm{N}$ & $\mathrm{P}$ & $\mathrm{S}$ & $\mathrm{S}$ & $\mathrm{S}$ & $\mathrm{S}$ & $\mathrm{P}$ & $\mathrm{N}$ & $\mathrm{N}$ & $\mathrm{N}$ & $\mathrm{N}$ \\
\hline Nova Luzitânia & $\mathrm{N}$ & $\mathrm{P}$ & $\mathrm{S}$ & $\mathrm{S}$ & $\mathrm{S}$ & $\mathrm{S}$ & $\mathrm{P}$ & $\mathrm{P}$ & $\mathrm{N}$ & $\mathrm{N}$ & $\mathrm{N}$ & $\mathrm{N}$ \\
\hline Nova Odessa & $\mathrm{P}$ & $\mathrm{S}$ & $\mathrm{S}$ & NR & NR & NR & $\mathrm{S}$ & $\mathrm{S}$ & $\mathrm{S}$ & $\mathrm{P}$ & $\mathrm{P}$ & $\mathrm{P}$ \\
\hline Novais & $\mathrm{P}$ & $\mathrm{S}$ & $\mathrm{S}$ & $\mathrm{S}$ & $\mathrm{S}$ & S & $\mathrm{S}$ & $\mathrm{P}$ & $\mathrm{P}$ & $\mathrm{P}$ & $\mathrm{P}$ & $\mathrm{P}$ \\
\hline Novo Horizonte & $\mathrm{P}$ & $\mathrm{P}$ & $\mathrm{S}$ & $\mathrm{S}$ & $\mathrm{S}$ & $\mathrm{S}$ & $\mathrm{S}$ & $\mathrm{P}$ & $\mathrm{P}$ & $\mathrm{P}$ & $\mathrm{P}$ & $\mathrm{N}$ \\
\hline Nuporanga & $\mathrm{P}$ & $\mathrm{S}$ & $\mathrm{S}$ & $\mathrm{S}$ & $\mathrm{S}$ & $\mathrm{S}$ & $\mathrm{S}$ & $\mathrm{S}$ & $\mathrm{P}$ & $\mathrm{P}$ & $\mathrm{P}$ & $\mathrm{P}$ \\
\hline Ocauçu & $\mathrm{P}$ & $\mathrm{S}$ & $\mathrm{S}$ & $\mathrm{S}$ & $\mathrm{S}$ & $\mathrm{S}$ & $\mathrm{S}$ & $\mathrm{S}$ & $\mathrm{S}$ & $\mathrm{P}$ & $\mathrm{P}$ & $\mathrm{P}$ \\
\hline Óleo & $\mathrm{P}$ & S & S & $\mathrm{S}$ & NR & $\mathrm{S}$ & $\mathrm{S}$ & $\mathrm{S}$ & $\mathrm{S}$ & $\mathrm{P}$ & $\mathrm{P}$ & $\mathrm{P}$ \\
\hline Olímpia & $\mathrm{P}$ & $\mathrm{P}$ & $\mathrm{S}$ & $\mathrm{S}$ & $\mathrm{S}$ & $\mathrm{S}$ & $\mathrm{S}$ & $\mathrm{P}$ & $\mathrm{P}$ & $\mathrm{P}$ & $\mathrm{P}$ & $\mathrm{N}$ \\
\hline
\end{tabular}


Tabela 10. Genótipos de milho mais recomendados para cada início de ciclo nos municípios do Estado de São Paulo, durante os doze meses do ano. N: normal; P: precoce; S: superprecoce; NR: não recomendável

\begin{tabular}{|c|c|c|c|c|c|c|c|c|c|c|c|c|}
\hline \multirow{2}{*}{ Município } & \multicolumn{12}{|c|}{ Meses } \\
\hline & Jan & Fev & Mar & Abr & Mai & Jun & Jul & Ago & Set & Out & Nov & Dez \\
\hline OndaVerde & $\mathrm{P}$ & $\mathrm{P}$ & $\mathrm{S}$ & $\mathrm{S}$ & $\mathrm{S}$ & $\mathrm{S}$ & $\mathrm{S}$ & $\mathrm{P}$ & $\mathrm{P}$ & $\mathrm{P}$ & $\mathrm{P}$ & $\mathrm{N}$ \\
\hline Oriente & $\mathrm{P}$ & $\mathrm{S}$ & $\mathrm{S}$ & NR & NR & $\mathrm{S}$ & $\mathrm{S}$ & $\mathrm{S}$ & $\mathrm{S}$ & $\mathrm{P}$ & $\mathrm{P}$ & $\mathrm{P}$ \\
\hline Orindiuva & $\mathrm{N}$ & $\mathrm{P}$ & $\mathrm{S}$ & $\mathrm{S}$ & $\mathrm{S}$ & $\mathrm{S}$ & $\mathrm{P}$ & $\mathrm{P}$ & $\mathrm{N}$ & $\mathrm{N}$ & $\mathrm{N}$ & $\mathrm{N}$ \\
\hline Orlândia & $\mathrm{P}$ & $\mathrm{S}$ & $\mathrm{S}$ & $\mathrm{S}$ & $\mathrm{S}$ & $\mathrm{S}$ & $\mathrm{S}$ & $\mathrm{S}$ & $\mathrm{S}$ & $\mathrm{P}$ & $\mathrm{P}$ & $\mathrm{P}$ \\
\hline Osasco & $\mathrm{S}$ & $\mathrm{S}$ & NR & NR & NR & NR & NR & $\mathrm{S}$ & $\mathrm{S}$ & $\mathrm{S}$ & $\mathrm{S}$ & $\mathrm{P}$ \\
\hline Oscar Bressane & $\mathrm{P}$ & $\mathrm{S}$ & $\mathrm{S}$ & $\mathrm{S}$ & $\mathrm{S}$ & $\mathrm{S}$ & $\mathrm{S}$ & $\mathrm{S}$ & $\mathrm{P}$ & $\mathrm{P}$ & $\mathrm{P}$ & $\mathrm{P}$ \\
\hline Oswaldo Cruz & $\mathrm{P}$ & $\mathrm{P}$ & $\mathrm{S}$ & $\mathrm{S}$ & $\mathrm{S}$ & $\mathrm{S}$ & $\mathrm{S}$ & $\mathrm{S}$ & $\mathrm{P}$ & $\mathrm{P}$ & $\mathrm{P}$ & $\mathrm{N}$ \\
\hline Ourinhos & $\mathrm{P}$ & $\mathrm{S}$ & $\mathrm{S}$ & NR & NR & $\mathrm{S}$ & $\mathrm{S}$ & $\mathrm{S}$ & $\mathrm{S}$ & $\mathrm{P}$ & $\mathrm{P}$ & $\mathrm{P}$ \\
\hline Ouro Verde & $\mathrm{N}$ & $\mathrm{P}$ & $\mathrm{P}$ & $\mathrm{S}$ & $\mathrm{S}$ & $\mathrm{S}$ & $\mathrm{S}$ & $\mathrm{P}$ & $\mathrm{N}$ & $\mathrm{N}$ & $\mathrm{N}$ & $\mathrm{N}$ \\
\hline Ouroeste & $\mathrm{N}$ & $\mathrm{N}$ & $\mathrm{P}$ & $\mathrm{S}$ & $\mathrm{S}$ & $\mathrm{S}$ & $\mathrm{P}$ & $\mathrm{N}$ & $\mathrm{N}$ & $\mathrm{N}$ & $\mathrm{N}$ & $\mathrm{N}$ \\
\hline Pacaembu & $\mathrm{N}$ & $\mathrm{P}$ & $\mathrm{P}$ & $\mathrm{S}$ & $\mathrm{S}$ & $\mathrm{S}$ & $\mathrm{S}$ & $\mathrm{P}$ & $\mathrm{N}$ & $\mathrm{N}$ & $\mathrm{N}$ & $\mathrm{N}$ \\
\hline Palestina & $\mathrm{P}$ & $\mathrm{P}$ & $\mathrm{S}$ & $\mathrm{S}$ & $\mathrm{S}$ & $\mathrm{S}$ & $\mathrm{S}$ & $\mathrm{P}$ & $\mathrm{P}$ & $\mathrm{P}$ & $\mathrm{P}$ & $\mathrm{P}$ \\
\hline Palmares Paulista & $\mathrm{P}$ & $\mathrm{P}$ & $\mathrm{S}$ & $\mathrm{S}$ & $\mathrm{S}$ & $\mathrm{S}$ & $\mathrm{S}$ & $\mathrm{P}$ & $\mathrm{P}$ & $\mathrm{P}$ & $\mathrm{P}$ & $\mathrm{P}$ \\
\hline Palmeira D'oeste & $\mathrm{N}$ & $\mathrm{P}$ & $\mathrm{P}$ & $\mathrm{S}$ & $\mathrm{S}$ & $\mathrm{S}$ & $\mathrm{P}$ & $\mathrm{P}$ & $\mathrm{N}$ & $\mathrm{N}$ & $\mathrm{N}$ & $\mathrm{N}$ \\
\hline Palmital & $\mathrm{P}$ & $\mathrm{S}$ & $\mathrm{S}$ & $\mathrm{S}$ & $\mathrm{S}$ & $\mathrm{S}$ & $\mathrm{S}$ & $\mathrm{S}$ & $\mathrm{P}$ & $\mathrm{P}$ & $\mathrm{P}$ & $\mathrm{P}$ \\
\hline Panorama & $\mathrm{N}$ & $\mathrm{P}$ & $\mathrm{P}$ & $\mathrm{S}$ & $\mathrm{S}$ & $\mathrm{S}$ & $\mathrm{S}$ & $\mathrm{P}$ & $\mathrm{N}$ & $\mathrm{N}$ & $\mathrm{N}$ & $\mathrm{N}$ \\
\hline Paraguassu Paulista & $\mathrm{P}$ & $\mathrm{S}$ & $\mathrm{S}$ & $\mathrm{S}$ & $\mathrm{S}$ & $\mathrm{S}$ & $\mathrm{S}$ & $\mathrm{S}$ & $\mathrm{S}$ & $\mathrm{P}$ & $\mathrm{P}$ & $\mathrm{P}$ \\
\hline Paraibuna & $\mathrm{S}$ & $\mathrm{S}$ & NR & NR & NR & NR & NR & $\mathrm{S}$ & $\mathrm{S}$ & $\mathrm{S}$ & $\mathrm{S}$ & $\mathrm{S}$ \\
\hline Paraíso & $\mathrm{P}$ & $\mathrm{P}$ & $\mathrm{S}$ & $\mathrm{S}$ & $\mathrm{S}$ & $\mathrm{S}$ & $\mathrm{S}$ & $\mathrm{P}$ & $\mathrm{P}$ & $\mathrm{P}$ & $\mathrm{P}$ & $\mathrm{P}$ \\
\hline Paranapanema & $\mathrm{S}$ & $\mathrm{S}$ & NR & NR & NR & NR & $\mathrm{S}$ & $\mathrm{S}$ & $\mathrm{S}$ & $\mathrm{S}$ & $\mathrm{S}$ & $\mathrm{P}$ \\
\hline Paranapuã & $\mathrm{N}$ & $\mathrm{N}$ & $\mathrm{P}$ & $\mathrm{S}$ & $\mathrm{S}$ & $\mathrm{S}$ & $\mathrm{P}$ & $\mathrm{N}$ & $\mathrm{N}$ & $\mathrm{N}$ & $\mathrm{N}$ & $\mathrm{N}$ \\
\hline Parapuã & $\mathrm{P}$ & $\mathrm{P}$ & $\mathrm{S}$ & $\mathrm{S}$ & $\mathrm{S}$ & $\mathrm{S}$ & $\mathrm{S}$ & $\mathrm{P}$ & $\mathrm{P}$ & $\mathrm{P}$ & $\mathrm{N}$ & $\mathrm{N}$ \\
\hline Pardinho & $\mathrm{S}$ & $\mathrm{S}$ & NR & NR & NR & NR & $\mathrm{S}$ & $\mathrm{S}$ & $\mathrm{S}$ & $\mathrm{S}$ & $\mathrm{S}$ & $\mathrm{P}$ \\
\hline Pariquera-Açu & $\mathrm{P}$ & $\mathrm{P}$ & $\mathrm{S}$ & NR & NR & NR & $\mathrm{S}$ & $\mathrm{S}$ & $\mathrm{S}$ & $\mathrm{P}$ & $\mathrm{P}$ & $\mathrm{N}$ \\
\hline Parisi & $\mathrm{N}$ & $\mathrm{P}$ & $\mathrm{S}$ & $\mathrm{S}$ & $\mathrm{S}$ & $\mathrm{S}$ & $\mathrm{P}$ & $\mathrm{P}$ & $\mathrm{N}$ & $\mathrm{N}$ & $\mathrm{N}$ & $\mathrm{N}$ \\
\hline Patrocínio Paulista & $\mathrm{P}$ & $\mathrm{S}$ & $\mathrm{S}$ & $\mathrm{S}$ & $\mathrm{S}$ & $\mathrm{S}$ & $\mathrm{S}$ & $\mathrm{S}$ & $\mathrm{S}$ & $\mathrm{P}$ & $\mathrm{P}$ & $\mathrm{P}$ \\
\hline Paulicéia & $\mathrm{N}$ & $\mathrm{N}$ & $\mathrm{P}$ & $\mathrm{S}$ & $\mathrm{S}$ & $\mathrm{S}$ & $\mathrm{S}$ & $\mathrm{P}$ & $\mathrm{N}$ & $\mathrm{N}$ & $\mathrm{N}$ & $\mathrm{N}$ \\
\hline Paulínia & $\mathrm{S}$ & $\mathrm{S}$ & $\mathrm{S}$ & NR & NR & NR & $\mathrm{S}$ & $\mathrm{S}$ & $\mathrm{S}$ & $\mathrm{S}$ & $\mathrm{P}$ & $\mathrm{P}$ \\
\hline Paulistania & $\mathrm{S}$ & $\mathrm{S}$ & $\mathrm{S}$ & NR & NR & NR & $\mathrm{S}$ & $\mathrm{S}$ & $\mathrm{S}$ & $\mathrm{S}$ & $\mathrm{P}$ & $\mathrm{P}$ \\
\hline Paulo de Faria & $\mathrm{N}$ & $\mathrm{P}$ & $\mathrm{S}$ & $\mathrm{S}$ & $\mathrm{S}$ & $\mathrm{S}$ & $\mathrm{P}$ & $\mathrm{P}$ & $\mathrm{N}$ & $\mathrm{N}$ & $\mathrm{N}$ & $\mathrm{N}$ \\
\hline Pederneiras & $\mathrm{P}$ & $\mathrm{S}$ & $\mathrm{S}$ & $\mathrm{S}$ & $\mathrm{S}$ & $\mathrm{S}$ & $\mathrm{S}$ & $\mathrm{S}$ & $\mathrm{P}$ & $\mathrm{P}$ & $\mathrm{P}$ & $\mathrm{P}$ \\
\hline Pedra Bela & $\mathrm{S}$ & $\mathrm{S}$ & NR & NR & NR & NR & NR & $\mathrm{S}$ & $\mathrm{S}$ & $\mathrm{S}$ & $\mathrm{S}$ & $\mathrm{S}$ \\
\hline Pedranópolis & $\mathrm{N}$ & $\mathrm{P}$ & $\mathrm{S}$ & $\mathrm{S}$ & $\mathrm{S}$ & $\mathrm{S}$ & $\mathrm{P}$ & $\mathrm{P}$ & $\mathrm{N}$ & $\mathrm{N}$ & $\mathrm{N}$ & $\mathrm{N}$ \\
\hline Pedregulho & $\mathrm{P}$ & $\mathrm{S}$ & $\mathrm{S}$ & $\mathrm{S}$ & $\mathrm{S}$ & $\mathrm{S}$ & $\mathrm{S}$ & $\mathrm{S}$ & $\mathrm{P}$ & $\mathrm{P}$ & $\mathrm{P}$ & $\mathrm{P}$ \\
\hline Pedreira & $\mathrm{P}$ & $\mathrm{S}$ & $\mathrm{S}$ & NR & NR & NR & $\mathrm{S}$ & $\mathrm{S}$ & $\mathrm{S}$ & $\mathrm{S}$ & $\mathrm{P}$ & $\mathrm{P}$ \\
\hline Pedrinhas Paulista & $\mathrm{P}$ & $\mathrm{P}$ & $\mathrm{S}$ & $\mathrm{S}$ & $\mathrm{S}$ & $\mathrm{S}$ & $\mathrm{S}$ & $\mathrm{S}$ & $\mathrm{P}$ & $\mathrm{P}$ & $\mathrm{N}$ & $\mathrm{N}$ \\
\hline Pedro de Toledo & $\mathrm{S}$ & $\mathrm{S}$ & NR & NR & NR & NR & NR & $\mathrm{S}$ & $\mathrm{S}$ & $\mathrm{S}$ & $\mathrm{S}$ & $\mathrm{S}$ \\
\hline Penápolis & $\mathrm{N}$ & $\mathrm{P}$ & $\mathrm{S}$ & $\mathrm{S}$ & $\mathrm{S}$ & $\mathrm{S}$ & $\mathrm{S}$ & $\mathrm{P}$ & $\mathrm{P}$ & $\mathrm{N}$ & $\mathrm{N}$ & $\mathrm{N}$ \\
\hline Pereira Barreto & $\mathrm{N}$ & $\mathrm{P}$ & $\mathrm{P}$ & $\mathrm{S}$ & $\mathrm{S}$ & $\mathrm{S}$ & $\mathrm{P}$ & $\mathrm{N}$ & $\mathrm{N}$ & $\mathrm{N}$ & $\mathrm{N}$ & $\mathrm{N}$ \\
\hline Pereiras & $\mathrm{P}$ & $\mathrm{S}$ & $\mathrm{S}$ & NR & NR & NR & $\mathrm{S}$ & $\mathrm{S}$ & $\mathrm{S}$ & $\mathrm{S}$ & $\mathrm{P}$ & $\mathrm{P}$ \\
\hline Peruíbe & $\mathrm{S}$ & NR & NR & NR & NR & NR & NR & NR & $\mathrm{S}$ & $\mathrm{S}$ & $\mathrm{S}$ & $\mathrm{S}$ \\
\hline Piacatu & $\mathrm{N}$ & P & $\mathrm{S}$ & $\mathrm{S}$ & $\mathrm{S}$ & $\mathrm{S}$ & $\mathrm{S}$ & $\mathrm{P}$ & $\mathrm{P}$ & $\mathrm{P}$ & $\mathrm{N}$ & $\mathrm{N}$ \\
\hline Piedade & $\mathrm{S}$ & $\mathrm{S}$ & NR & NR & NR & NR & $\mathrm{S}$ & $\mathrm{S}$ & $\mathrm{S}$ & $\mathrm{S}$ & $\mathrm{P}$ & $\mathrm{P}$ \\
\hline
\end{tabular}


Tabela 10. Genótipos de milho mais recomendados para cada início de ciclo nos municípios do Estado de São Paulo, durante os doze meses do ano. N: normal; P: precoce; S: superprecoce; NR: não recomendável

\begin{tabular}{|c|c|c|c|c|c|c|c|c|c|c|c|c|}
\hline \multirow{2}{*}{ Município } & \multicolumn{12}{|c|}{ Meses } \\
\hline & Jan & $\mathrm{Fev}$ & Mar & Abr & Mai & Jun & Jul & Ago & Set & Out & Nov & Dez \\
\hline Pilar do Sul & $\mathrm{S}$ & NR & NR & NR & NR & NR & NR & NR & $\mathrm{S}$ & $\mathrm{S}$ & $\mathrm{S}$ & $\mathrm{S}$ \\
\hline Pindamonhangaba & $\mathrm{P}$ & $\mathrm{S}$ & $\mathrm{S}$ & NR & NR & NR & $\mathrm{S}$ & $\mathrm{S}$ & $\mathrm{S}$ & $\mathrm{P}$ & $\mathrm{P}$ & $\mathrm{P}$ \\
\hline Pindorama & $\mathrm{P}$ & $\mathrm{S}$ & $\mathrm{S}$ & $\mathrm{S}$ & $\mathrm{S}$ & $\mathrm{S}$ & $\mathrm{S}$ & $\mathrm{S}$ & $\mathrm{P}$ & $\mathrm{P}$ & $\mathrm{P}$ & $\mathrm{P}$ \\
\hline Pinhalzinho & $\mathrm{S}$ & NR & NR & NR & NR & NR & NR & NR & $\mathrm{S}$ & $\mathrm{S}$ & $\mathrm{S}$ & $\mathrm{S}$ \\
\hline Piquerobi & $\mathrm{N}$ & $\mathrm{P}$ & $\mathrm{S}$ & $\mathrm{S}$ & $\mathrm{S}$ & $\mathrm{S}$ & $\mathrm{S}$ & $\mathrm{P}$ & $\mathrm{P}$ & $\mathrm{N}$ & $\mathrm{N}$ & $\mathrm{N}$ \\
\hline Piquete & $\mathrm{S}$ & NR & NR & NR & NR & NR & NR & $\mathrm{S}$ & $\mathrm{S}$ & $\mathrm{S}$ & $\mathrm{S}$ & $\mathrm{S}$ \\
\hline Piracaia & $\mathrm{S}$ & NR & NR & NR & NR & NR & NR & NR & $\mathrm{S}$ & $\mathrm{S}$ & $\mathrm{S}$ & $\mathrm{S}$ \\
\hline Piracicaba & $\mathrm{P}$ & $\mathrm{S}$ & $\mathrm{S}$ & NR & NR & NR & $\mathrm{S}$ & $\mathrm{S}$ & $\mathrm{S}$ & $\mathrm{S}$ & $\mathrm{P}$ & $\mathrm{P}$ \\
\hline Piraju & $\mathrm{S}$ & $\mathrm{S}$ & NR & NR & NR & NR & $\mathrm{S}$ & $\mathrm{S}$ & $\mathrm{S}$ & $\mathrm{S}$ & $\mathrm{P}$ & $\mathrm{P}$ \\
\hline Pirajuí & $\mathrm{P}$ & $\mathrm{S}$ & $\mathrm{S}$ & $\mathrm{S}$ & $\mathrm{S}$ & $\mathrm{S}$ & $\mathrm{S}$ & $\mathrm{S}$ & $\mathrm{P}$ & $\mathrm{P}$ & $\mathrm{P}$ & $\mathrm{P}$ \\
\hline Pirangi & $\mathrm{P}$ & $\mathrm{P}$ & $\mathrm{S}$ & $\mathrm{S}$ & $\mathrm{S}$ & $\mathrm{S}$ & $\mathrm{S}$ & $\mathrm{P}$ & $\mathrm{P}$ & $\mathrm{P}$ & $\mathrm{P}$ & $\mathrm{P}$ \\
\hline Pirapora do Bom Jesus & $\mathrm{P}$ & $\mathrm{S}$ & $\mathrm{S}$ & NR & NR & NR & $\mathrm{S}$ & $\mathrm{S}$ & $\mathrm{S}$ & $\mathrm{S}$ & $\mathrm{P}$ & $\mathrm{P}$ \\
\hline Pirapozinho & $\mathrm{N}$ & $\mathrm{P}$ & $\mathrm{S}$ & $\mathrm{S}$ & $\mathrm{S}$ & $\mathrm{S}$ & $\mathrm{S}$ & $\mathrm{P}$ & $\mathrm{P}$ & $\mathrm{N}$ & $\mathrm{N}$ & $\mathrm{N}$ \\
\hline Pirassununga & $\mathrm{P}$ & $\mathrm{S}$ & $\mathrm{S}$ & NR & NR & $\mathrm{S}$ & $\mathrm{S}$ & $\mathrm{S}$ & $\mathrm{S}$ & $\mathrm{S}$ & $\mathrm{P}$ & $\mathrm{P}$ \\
\hline Piratininga & $\mathrm{P}$ & $\mathrm{S}$ & $\mathrm{S}$ & NR & NR & $\mathrm{S}$ & $\mathrm{S}$ & $\mathrm{S}$ & $\mathrm{S}$ & $\mathrm{P}$ & $\mathrm{P}$ & $\mathrm{P}$ \\
\hline Pitangueiras & $\mathrm{P}$ & $\mathrm{S}$ & $\mathrm{S}$ & $\mathrm{S}$ & $\mathrm{S}$ & $\mathrm{S}$ & $\mathrm{S}$ & $\mathrm{S}$ & $\mathrm{P}$ & $\mathrm{P}$ & $\mathrm{P}$ & $\mathrm{P}$ \\
\hline Planalto & $\mathrm{N}$ & $\mathrm{P}$ & $\mathrm{S}$ & $\mathrm{S}$ & $\mathrm{S}$ & $\mathrm{S}$ & $\mathrm{S}$ & $\mathrm{P}$ & $\mathrm{P}$ & $\mathrm{N}$ & $\mathrm{N}$ & $\mathrm{N}$ \\
\hline Platina & $\mathrm{P}$ & $\mathrm{S}$ & $\mathrm{S}$ & $\mathrm{S}$ & $\mathrm{S}$ & $\mathrm{S}$ & $\mathrm{S}$ & $\mathrm{S}$ & $\mathrm{S}$ & $\mathrm{P}$ & $\mathrm{P}$ & $\mathrm{P}$ \\
\hline Poá & $\mathrm{S}$ & $\mathrm{S}$ & NR & NR & NR & NR & NR & $\mathrm{S}$ & $\mathrm{S}$ & $\mathrm{S}$ & $\mathrm{S}$ & $\mathrm{S}$ \\
\hline Poloni & $\mathrm{P}$ & $\mathrm{P}$ & $\mathrm{S}$ & $\mathrm{S}$ & $\mathrm{S}$ & $\mathrm{S}$ & $\mathrm{S}$ & $\mathrm{P}$ & $\mathrm{P}$ & $\mathrm{N}$ & $\mathrm{N}$ & $\mathrm{N}$ \\
\hline Pompéia & $\mathrm{P}$ & $\mathrm{P}$ & $\mathrm{S}$ & $\mathrm{S}$ & $\mathrm{S}$ & $\mathrm{S}$ & $\mathrm{S}$ & $\mathrm{S}$ & $\mathrm{P}$ & $\mathrm{P}$ & $\mathrm{P}$ & $\mathrm{P}$ \\
\hline Pongai & $\mathrm{P}$ & $\mathrm{P}$ & $\mathrm{S}$ & $\mathrm{S}$ & $\mathrm{S}$ & $\mathrm{S}$ & $\mathrm{S}$ & $\mathrm{P}$ & $\mathrm{P}$ & $\mathrm{P}$ & $\mathrm{N}$ & $\mathrm{N}$ \\
\hline Pontal & $\mathrm{P}$ & $\mathrm{S}$ & $\mathrm{S}$ & $\mathrm{S}$ & $\mathrm{S}$ & $\mathrm{S}$ & $\mathrm{S}$ & $\mathrm{S}$ & $\mathrm{P}$ & $\mathrm{P}$ & $\mathrm{P}$ & $\mathrm{P}$ \\
\hline Pontalinda & $\mathrm{N}$ & $\mathrm{P}$ & $\mathrm{P}$ & $\mathrm{S}$ & $\mathrm{S}$ & $\mathrm{S}$ & $P$ & $\mathrm{P}$ & $\mathrm{N}$ & $\mathrm{N}$ & $\mathrm{N}$ & $\mathrm{N}$ \\
\hline Pontes Gestal & $\mathrm{N}$ & $\mathrm{P}$ & $\mathrm{S}$ & $\mathrm{S}$ & $\mathrm{S}$ & $\mathrm{S}$ & $\mathrm{S}$ & $\mathrm{P}$ & $\mathrm{N}$ & $\mathrm{N}$ & $\mathrm{N}$ & $\mathrm{N}$ \\
\hline Populina & $\mathrm{N}$ & $\mathrm{N}$ & $\mathrm{P}$ & $\mathrm{S}$ & $\mathrm{S}$ & $\mathrm{S}$ & $\mathrm{P}$ & $\mathrm{N}$ & $\mathrm{N}$ & $\mathrm{N}$ & $\mathrm{N}$ & $\mathrm{N}$ \\
\hline Porangaba & $\mathrm{S}$ & $\mathrm{S}$ & NR & NR & NR & NR & $\mathrm{S}$ & $\mathrm{S}$ & $\mathrm{S}$ & $\mathrm{S}$ & $\mathrm{S}$ & $\mathrm{S}$ \\
\hline Porto Feliz & $\mathrm{P}$ & $\mathrm{S}$ & $\mathrm{S}$ & NR & NR & NR & $\mathrm{S}$ & $\mathrm{S}$ & $\mathrm{S}$ & $\mathrm{P}$ & $\mathrm{P}$ & $\mathrm{P}$ \\
\hline Porto Ferreira & $\mathrm{S}$ & $\mathrm{S}$ & NR & NR & NR & NR & $\mathrm{S}$ & $\mathrm{S}$ & $\mathrm{S}$ & $\mathrm{S}$ & $\mathrm{S}$ & $\mathrm{S}$ \\
\hline Potim & NR & NR & NR & NR & NR & NR & NR & NR & NR & NR & NR & NR \\
\hline Potirendaba & $\mathrm{P}$ & $P$ & $\mathrm{~S}$ & $\mathrm{~S}$ & $\mathrm{~S}$ & $\mathrm{~S}$ & $\mathrm{~S}$ & $\mathrm{P}$ & $\mathrm{P}$ & $\mathrm{P}$ & $\mathrm{P}$ & $\mathrm{N}$ \\
\hline Pracinha & $\mathrm{N}$ & $\mathrm{P}$ & $\mathrm{S}$ & $\mathrm{S}$ & $\mathrm{S}$ & $\mathrm{S}$ & $\mathrm{S}$ & $\mathrm{P}$ & $\mathrm{P}$ & $\mathrm{N}$ & $\mathrm{N}$ & $\mathrm{N}$ \\
\hline Pradópolis & $\mathrm{P}$ & $\mathrm{S}$ & $\mathrm{S}$ & $\mathrm{S}$ & $\mathrm{S}$ & $\mathrm{S}$ & $\mathrm{S}$ & $\mathrm{S}$ & $\mathrm{P}$ & $\mathrm{P}$ & $\mathrm{P}$ & $\mathrm{P}$ \\
\hline Praia Grande & $\mathrm{S}$ & NR & NR & NR & NR & NR & NR & NR & NR & $\mathrm{S}$ & $\mathrm{S}$ & $\mathrm{S}$ \\
\hline Pratânia & $\mathrm{P}$ & $\mathrm{S}$ & $\mathrm{S}$ & NR & NR & $\mathrm{S}$ & $\mathrm{S}$ & $\mathrm{S}$ & $\mathrm{S}$ & $\mathrm{P}$ & $\mathrm{P}$ & $\mathrm{P}$ \\
\hline Presidente Alves & $\mathrm{P}$ & $\mathrm{P}$ & $\mathrm{S}$ & $\mathrm{S}$ & $\mathrm{S}$ & $\mathrm{S}$ & $\mathrm{S}$ & $\mathrm{S}$ & $\mathrm{P}$ & $\mathrm{P}$ & $\mathrm{P}$ & $\mathrm{N}$ \\
\hline Presidente Bernardes & $\mathrm{N}$ & $\mathrm{P}$ & $\mathrm{S}$ & $\mathrm{S}$ & $\mathrm{S}$ & $\mathrm{S}$ & $\mathrm{S}$ & $\mathrm{P}$ & $\mathrm{P}$ & $\mathrm{N}$ & $\mathrm{N}$ & $\mathrm{N}$ \\
\hline Presidente Epitácio & $\mathrm{N}$ & $\mathrm{P}$ & $\mathrm{S}$ & $\mathrm{S}$ & $\mathrm{S}$ & $\mathrm{S}$ & $\mathrm{S}$ & $\mathrm{P}$ & $\mathrm{P}$ & $\mathrm{N}$ & $\mathrm{N}$ & $\mathrm{N}$ \\
\hline Presidente Prudente & $\mathrm{P}$ & $\mathrm{S}$ & $\mathrm{S}$ & $\mathrm{S}$ & $\mathrm{S}$ & $\mathrm{S}$ & $\mathrm{S}$ & $\mathrm{S}$ & $\mathrm{P}$ & $\mathrm{P}$ & $\mathrm{P}$ & $\mathrm{P}$ \\
\hline Presidente Venceslau & $\mathrm{P}$ & $\mathrm{P}$ & $\mathrm{S}$ & $\mathrm{S}$ & $\mathrm{S}$ & $\mathrm{S}$ & $\mathrm{S}$ & $\mathrm{S}$ & $\mathrm{P}$ & $\mathrm{P}$ & $\mathrm{N}$ & $\mathrm{N}$ \\
\hline Promissão & $\mathrm{N}$ & $\mathrm{P}$ & $\mathrm{S}$ & $\mathrm{S}$ & $\mathrm{S}$ & $\mathrm{S}$ & $\mathrm{S}$ & $\mathrm{P}$ & $\mathrm{P}$ & $\mathrm{N}$ & $\mathrm{N}$ & $\mathrm{N}$ \\
\hline Quadra & $\mathrm{S}$ & $\mathrm{S}$ & NR & NR & NR & NR & $\mathrm{S}$ & $\mathrm{S}$ & $\mathrm{S}$ & $\mathrm{S}$ & $\mathrm{S}$ & $\mathrm{P}$ \\
\hline Quatá & $\mathrm{P}$ & $\mathrm{P}$ & $\mathrm{S}$ & $\mathrm{S}$ & $\mathrm{S}$ & $\mathrm{S}$ & $\mathrm{S}$ & $\mathrm{S}$ & $\mathrm{P}$ & $\mathrm{P}$ & $\mathrm{P}$ & $\mathrm{P}$ \\
\hline
\end{tabular}


Tabela 10. Genótipos de milho mais recomendados para cada início de ciclo nos municípios do Estado de São Paulo, durante os doze meses do ano. N: normal; P: precoce; S: superprecoce; NR: não recomendável

\begin{tabular}{|c|c|c|c|c|c|c|c|c|c|c|c|c|}
\hline \multirow{2}{*}{ Município } & \multicolumn{12}{|c|}{ Meses } \\
\hline & Jan & Fev & Mar & Abr & Mai & Jun & Jul & Ago & Set & Out & Nov & Dez \\
\hline Queiroz & $\mathrm{N}$ & $\mathrm{P}$ & $\mathrm{S}$ & $\mathrm{S}$ & $\mathrm{S}$ & $\mathrm{S}$ & $\mathrm{S}$ & $\mathrm{P}$ & $\mathrm{P}$ & $\mathrm{P}$ & $\mathrm{N}$ & $\mathrm{N}$ \\
\hline Queluz & $\mathrm{P}$ & $\mathrm{S}$ & $\mathrm{S}$ & NR & NR & NR & $\mathrm{S}$ & $\mathrm{S}$ & $\mathrm{S}$ & $\mathrm{S}$ & $\mathrm{P}$ & $\mathrm{P}$ \\
\hline Quintana & $\mathrm{P}$ & $\mathrm{S}$ & $\mathrm{S}$ & NR & NR & $\mathrm{S}$ & $\mathrm{S}$ & $\mathrm{S}$ & $\mathrm{S}$ & $\mathrm{P}$ & $\mathrm{P}$ & $\mathrm{P}$ \\
\hline Rafard & $\mathrm{P}$ & $\mathrm{S}$ & $\mathrm{S}$ & NR & NR & NR & $\mathrm{S}$ & $\mathrm{S}$ & $\mathrm{S}$ & $\mathrm{S}$ & $\mathrm{P}$ & $\mathrm{P}$ \\
\hline Rancharia & $\mathrm{P}$ & $\mathrm{S}$ & $\mathrm{S}$ & $\mathrm{S}$ & $\mathrm{S}$ & S & $\mathrm{S}$ & $\mathrm{S}$ & $\mathrm{S}$ & $\mathrm{P}$ & $\mathrm{P}$ & $\mathrm{P}$ \\
\hline Redençãao da Serra & $\mathrm{S}$ & NR & NR & NR & NR & NR & NR & $\mathrm{S}$ & $\mathrm{S}$ & $\mathrm{S}$ & $\mathrm{S}$ & $\mathrm{S}$ \\
\hline Regente Feijó & $\mathrm{N}$ & $\mathrm{P}$ & $\mathrm{S}$ & $\mathrm{S}$ & $\mathrm{S}$ & $\mathrm{S}$ & $\mathrm{S}$ & $\mathrm{P}$ & $\mathrm{P}$ & $\mathrm{P}$ & $\mathrm{N}$ & $\mathrm{N}$ \\
\hline Reginópolis & $\mathrm{P}$ & $\mathrm{P}$ & $\mathrm{S}$ & $\mathrm{S}$ & $\mathrm{S}$ & $\mathrm{S}$ & $\mathrm{S}$ & $\mathrm{S}$ & $\mathrm{P}$ & $\mathrm{P}$ & $\mathrm{N}$ & $\mathrm{N}$ \\
\hline Registro & $\mathrm{N}$ & $\mathrm{N}$ & $\mathrm{S}$ & $\mathrm{S}$ & $\mathrm{S}$ & $\mathrm{S}$ & $\mathrm{S}$ & $\mathrm{P}$ & $\mathrm{P}$ & $\mathrm{N}$ & $\mathrm{N}$ & $\mathrm{N}$ \\
\hline Restinga & $\mathrm{P}$ & $\mathrm{S}$ & $\mathrm{S}$ & $\mathrm{S}$ & $\mathrm{S}$ & $\mathrm{S}$ & $\mathrm{S}$ & $\mathrm{P}$ & $\mathrm{P}$ & $\mathrm{P}$ & $\mathrm{P}$ & $\mathrm{P}$ \\
\hline Ribeira & $\mathrm{N}$ & $\mathrm{P}$ & $\mathrm{S}$ & $\mathrm{S}$ & $\mathrm{S}$ & $\mathrm{S}$ & $\mathrm{S}$ & $\mathrm{S}$ & $\mathrm{P}$ & $\mathrm{P}$ & $\mathrm{N}$ & $\mathrm{N}$ \\
\hline Ribeirão Bonito & $\mathrm{P}$ & $\mathrm{S}$ & $\mathrm{S}$ & NR & NR & $\mathrm{S}$ & $\mathrm{S}$ & $\mathrm{S}$ & $\mathrm{S}$ & $\mathrm{P}$ & $\mathrm{P}$ & $\mathrm{P}$ \\
\hline Ribeirão Branco & $\mathrm{S}$ & $\mathrm{S}$ & NR & NR & NR & NR & NR & $\mathrm{S}$ & $\mathrm{S}$ & $\mathrm{S}$ & $\mathrm{S}$ & $\mathrm{S}$ \\
\hline Ribeirão Corrente & $\mathrm{P}$ & $\mathrm{S}$ & $\mathrm{S}$ & $\mathrm{S}$ & $\mathrm{S}$ & $\mathrm{S}$ & $\mathrm{S}$ & $\mathrm{P}$ & $\mathrm{P}$ & $\mathrm{P}$ & $\mathrm{P}$ & $\mathrm{P}$ \\
\hline Ribeirão do Sul & $\mathrm{P}$ & $\mathrm{S}$ & $\mathrm{S}$ & $\mathrm{S}$ & NR & $\mathrm{S}$ & $\mathrm{S}$ & $\mathrm{S}$ & $\mathrm{S}$ & $\mathrm{P}$ & $\mathrm{P}$ & $\mathrm{P}$ \\
\hline Ribeirão dos Índios & $\mathrm{N}$ & $\mathrm{P}$ & $\mathrm{S}$ & $\mathrm{S}$ & $\mathrm{S}$ & $\mathrm{S}$ & $\mathrm{S}$ & $\mathrm{P}$ & $\mathrm{N}$ & $\mathrm{N}$ & $\mathrm{N}$ & $\mathrm{N}$ \\
\hline Ribeirão Grande & $\mathrm{S}$ & $\mathrm{S}$ & NR & NR & NR & NR & NR & $\mathrm{S}$ & $\mathrm{S}$ & $\mathrm{S}$ & $\mathrm{S}$ & $\mathrm{S}$ \\
\hline Ribeirão Pires & $\mathrm{S}$ & $\mathrm{S}$ & NR & NR & NR & NR & NR & $\mathrm{S}$ & $\mathrm{S}$ & $\mathrm{S}$ & $\mathrm{S}$ & $\mathrm{S}$ \\
\hline Ribeirão Preto & $\mathrm{S}$ & $\mathrm{S}$ & NR & NR & NR & NR & NR & $\mathrm{S}$ & $\mathrm{S}$ & $\mathrm{S}$ & $\mathrm{S}$ & $\mathrm{S}$ \\
\hline Rifaina & $\mathrm{S}$ & $\mathrm{S}$ & $\mathrm{S}$ & $\mathrm{S}$ & $\mathrm{S}$ & $\mathrm{S}$ & $\mathrm{S}$ & $\mathrm{S}$ & $\mathrm{P}$ & $\mathrm{P}$ & $\mathrm{P}$ & $\mathrm{P}$ \\
\hline Rincão & $\mathrm{P}$ & $\mathrm{S}$ & $\mathrm{S}$ & $\mathrm{S}$ & $\mathrm{S}$ & $\mathrm{S}$ & $\mathrm{S}$ & $\mathrm{S}$ & $\mathrm{S}$ & $\mathrm{P}$ & $\mathrm{P}$ & $\mathrm{P}$ \\
\hline Rinópolis & $\mathrm{N}$ & $\mathrm{P}$ & $\mathrm{S}$ & $\mathrm{S}$ & $\mathrm{S}$ & $\mathrm{S}$ & $\mathrm{S}$ & $\mathrm{P}$ & $\mathrm{P}$ & $\mathrm{P}$ & $\mathrm{N}$ & $\mathrm{N}$ \\
\hline Rio Claro & $P$ & $\mathrm{~S}$ & $\mathrm{~S}$ & NR & NR & NR & $\mathrm{S}$ & $\mathrm{S}$ & $\mathrm{S}$ & $\mathrm{S}$ & $\mathrm{P}$ & $\mathrm{P}$ \\
\hline Rio das Pedras & $\mathrm{S}$ & $\mathrm{S}$ & NR & NR & NR & NR & $\mathrm{S}$ & $\mathrm{S}$ & $\mathrm{S}$ & $\mathrm{S}$ & $\mathrm{S}$ & $\mathrm{P}$ \\
\hline Rio Grande da Serra & $\mathrm{S}$ & $\mathrm{S}$ & NR & NR & NR & NR & NR & $\mathrm{S}$ & $\mathrm{S}$ & $\mathrm{S}$ & $\mathrm{S}$ & $\mathrm{S}$ \\
\hline Riolândia & $\mathrm{N}$ & $\mathrm{P}$ & $\mathrm{S}$ & $\mathrm{S}$ & $\mathrm{S}$ & $\mathrm{S}$ & $\mathrm{P}$ & $\mathrm{P}$ & $\mathrm{N}$ & $\mathrm{N}$ & $\mathrm{N}$ & $\mathrm{N}$ \\
\hline Riversul & $\mathrm{P}$ & $\mathrm{S}$ & NR & NR & NR & NR & S & $\mathrm{S}$ & $\mathrm{S}$ & $\mathrm{S}$ & $\mathrm{P}$ & $\mathrm{P}$ \\
\hline Rosana & $\mathrm{P}$ & $\mathrm{S}$ & $\mathrm{S}$ & $\mathrm{S}$ & $\mathrm{S}$ & $\mathrm{S}$ & $\mathrm{S}$ & $\mathrm{S}$ & $\mathrm{P}$ & $\mathrm{P}$ & $\mathrm{P}$ & $\mathrm{P}$ \\
\hline Roseira & $\mathrm{P}$ & $\mathrm{S}$ & $\mathrm{S}$ & NR & NR & NR & $\mathrm{S}$ & $\mathrm{S}$ & $\mathrm{S}$ & $\mathrm{S}$ & $\mathrm{P}$ & $\mathrm{P}$ \\
\hline Rubiácea & $\mathrm{N}$ & $\mathrm{P}$ & $\mathrm{S}$ & $\mathrm{S}$ & $\mathrm{S}$ & $\mathrm{S}$ & $\mathrm{S}$ & $\mathrm{P}$ & $\mathrm{P}$ & $\mathrm{N}$ & $\mathrm{N}$ & $\mathrm{N}$ \\
\hline Rubineia & $\mathrm{N}$ & $\mathrm{N}$ & $\mathrm{P}$ & $\mathrm{S}$ & $\mathrm{S}$ & $\mathrm{S}$ & $\mathrm{P}$ & $\mathrm{N}$ & $\mathrm{N}$ & $\mathrm{N}$ & $\mathrm{N}$ & $\mathrm{N}$ \\
\hline Sabino & $\mathrm{N}$ & $\mathrm{P}$ & $\mathrm{S}$ & $\mathrm{S}$ & $\mathrm{S}$ & $\mathrm{S}$ & $\mathrm{S}$ & $\mathrm{P}$ & $\mathrm{P}$ & $\mathrm{N}$ & $\mathrm{N}$ & $\mathrm{N}$ \\
\hline Sagres & $\mathrm{N}$ & $\mathrm{P}$ & $\mathrm{S}$ & $\mathrm{S}$ & $\mathrm{S}$ & $\mathrm{S}$ & $\mathrm{S}$ & $\mathrm{P}$ & $\mathrm{P}$ & $\mathrm{N}$ & $\mathrm{N}$ & $\mathrm{N}$ \\
\hline Sales & $\mathrm{N}$ & $\mathrm{P}$ & $\mathrm{S}$ & $\mathrm{S}$ & $\mathrm{S}$ & $\mathrm{S}$ & $\mathrm{S}$ & $\mathrm{P}$ & $\mathrm{P}$ & $\mathrm{P}$ & $\mathrm{N}$ & $\mathrm{N}$ \\
\hline Sales Oliveira & $P$ & $\mathrm{~S}$ & $\mathrm{~S}$ & $\mathrm{~S}$ & $\mathrm{~S}$ & $\mathrm{~S}$ & $\mathrm{~S}$ & $\mathrm{P}$ & $\mathrm{P}$ & $\mathrm{P}$ & $\mathrm{P}$ & $\mathrm{P}$ \\
\hline Salesópolis & $\mathrm{S}$ & NR & NR & NR & NR & NR & NR & NR & $\mathrm{S}$ & $\mathrm{S}$ & $\mathrm{S}$ & $\mathrm{S}$ \\
\hline Salmourão & $\mathrm{N}$ & $\mathrm{P}$ & $\mathrm{S}$ & $\mathrm{S}$ & $\mathrm{S}$ & $\mathrm{S}$ & $\mathrm{S}$ & $\mathrm{P}$ & $\mathrm{P}$ & $\mathrm{N}$ & $\mathrm{N}$ & $\mathrm{N}$ \\
\hline Saltinho & $\mathrm{S}$ & $\mathrm{S}$ & NR & NR & NR & NR & $\mathrm{S}$ & $\mathrm{S}$ & $\mathrm{S}$ & $\mathrm{S}$ & $\mathrm{S}$ & $\mathrm{S}$ \\
\hline Salto & $\mathrm{P}$ & $\mathrm{S}$ & $\mathrm{S}$ & NR & NR & NR & $\mathrm{S}$ & $\mathrm{S}$ & $\mathrm{S}$ & $\mathrm{P}$ & $\mathrm{P}$ & $\mathrm{P}$ \\
\hline Salto de Pirapora & $\mathrm{P}$ & $\mathrm{S}$ & $\mathrm{S}$ & NR & NR & NR & $\mathrm{S}$ & $\mathrm{S}$ & $\mathrm{S}$ & $\mathrm{S}$ & $\mathrm{P}$ & $\mathrm{P}$ \\
\hline Salto Grande & $\mathrm{P}$ & $\mathrm{P}$ & $\mathrm{S}$ & $\mathrm{S}$ & $\mathrm{S}$ & $\mathrm{S}$ & $\mathrm{S}$ & $\mathrm{S}$ & $\mathrm{P}$ & $\mathrm{P}$ & $\mathrm{N}$ & $\mathrm{N}$ \\
\hline Sandovalina & $\mathrm{N}$ & $\mathrm{P}$ & $\mathrm{S}$ & $\mathrm{S}$ & $\mathrm{S}$ & $\mathrm{S}$ & $\mathrm{S}$ & $\mathrm{P}$ & $\mathrm{P}$ & $\mathrm{N}$ & $\mathrm{N}$ & $\mathrm{N}$ \\
\hline Santa Adélia & $\mathrm{P}$ & $\mathrm{S}$ & $\mathrm{S}$ & $\mathrm{S}$ & $\mathrm{S}$ & $\mathrm{S}$ & $\mathrm{S}$ & $\mathrm{S}$ & $\mathrm{S}$ & $\mathrm{P}$ & $\mathrm{P}$ & $\mathrm{P}$ \\
\hline
\end{tabular}


Tabela 10. Genótipos de milho mais recomendados para cada início de ciclo nos municípios do Estado de São Paulo, durante os doze meses do ano. N: normal; P: precoce; S: superprecoce; NR: não recomendável

\begin{tabular}{|c|c|c|c|c|c|c|c|c|c|c|c|c|}
\hline \multirow{2}{*}{ Município } & \multicolumn{12}{|c|}{ Meses } \\
\hline & Jan & $\mathrm{Fev}$ & Mar & Abr & Mai & Jun & Jul & Ago & Set & Out & Nov & Dez \\
\hline Santa Albertina & $\mathrm{N}$ & $\mathrm{N}$ & $\mathrm{P}$ & $\mathrm{S}$ & $\mathrm{S}$ & $\mathrm{S}$ & $P$ & $\mathrm{~N}$ & $\mathrm{~N}$ & $\mathrm{~N}$ & $\mathrm{~N}$ & $\mathrm{~N}$ \\
\hline Santa Bárbara D’oeste & $\mathrm{P}$ & $\mathrm{S}$ & $\mathrm{S}$ & NR & NR & $\mathrm{S}$ & $\mathrm{S}$ & $\mathrm{S}$ & $\mathrm{S}$ & $\mathrm{P}$ & $\mathrm{P}$ & $\mathrm{P}$ \\
\hline Santa Branca & $\mathrm{S}$ & $\mathrm{S}$ & NR & NR & NR & NR & NR & $\mathrm{S}$ & $\mathrm{S}$ & $\mathrm{S}$ & $\mathrm{S}$ & $\mathrm{S}$ \\
\hline Santa Clara D'oeste & $\mathrm{N}$ & $\mathrm{N}$ & $\mathrm{P}$ & $\mathrm{S}$ & $\mathrm{S}$ & $\mathrm{S}$ & $\mathrm{P}$ & $\mathrm{N}$ & $\mathrm{N}$ & $\mathrm{N}$ & $\mathrm{N}$ & $\mathrm{N}$ \\
\hline S. Cruz da Conceição & $\mathrm{S}$ & $\mathrm{S}$ & NR & NR & NR & NR & $\mathrm{S}$ & $\mathrm{S}$ & $\mathrm{S}$ & $\mathrm{S}$ & $\mathrm{S}$ & $\mathrm{S}$ \\
\hline S. Cruz da Esperança & $\mathrm{S}$ & $\mathrm{S}$ & $\mathrm{S}$ & NR & NR & NR & $\mathrm{S}$ & $\mathrm{S}$ & $\mathrm{S}$ & $\mathrm{S}$ & $\mathrm{S}$ & $\mathrm{S}$ \\
\hline S. Cruz das Palmeiras & $\mathrm{S}$ & $\mathrm{S}$ & $\mathrm{S}$ & NR & NR & $\mathrm{S}$ & $\mathrm{S}$ & $\mathrm{S}$ & $\mathrm{S}$ & $\mathrm{S}$ & $\mathrm{P}$ & $\mathrm{P}$ \\
\hline S. Cruz do Rio Pardo & $\mathrm{P}$ & $\mathrm{S}$ & $\mathrm{S}$ & NR & NR & $\mathrm{S}$ & $\mathrm{S}$ & $\mathrm{S}$ & $\mathrm{S}$ & $\mathrm{P}$ & $\mathrm{P}$ & $\mathrm{P}$ \\
\hline Santa Ernestina & $\mathrm{P}$ & $\mathrm{S}$ & $\mathrm{S}$ & $\mathrm{S}$ & $\mathrm{S}$ & $\mathrm{S}$ & $\mathrm{S}$ & $\mathrm{S}$ & $\mathrm{P}$ & $\mathrm{P}$ & $\mathrm{P}$ & $\mathrm{P}$ \\
\hline Santa Fé do Sul & $\mathrm{N}$ & $\mathrm{N}$ & $\mathrm{P}$ & $\mathrm{S}$ & $\mathrm{S}$ & $\mathrm{S}$ & $\mathrm{P}$ & $\mathrm{N}$ & $\mathrm{N}$ & $\mathrm{N}$ & $\mathrm{N}$ & $\mathrm{N}$ \\
\hline Santa Gertrudes & $\mathrm{S}$ & $\mathrm{S}$ & NR & NR & NR & NR & NR & $\mathrm{S}$ & $\mathrm{S}$ & $\mathrm{S}$ & $\mathrm{S}$ & $\mathrm{S}$ \\
\hline Santa Isabel & $\mathrm{S}$ & $\mathrm{S}$ & NR & NR & NR & NR & NR & $\mathrm{S}$ & $\mathrm{S}$ & $\mathrm{S}$ & $\mathrm{S}$ & $\mathrm{S}$ \\
\hline Santa Lúcia & $\mathrm{P}$ & $\mathrm{S}$ & $\mathrm{S}$ & $\mathrm{S}$ & $\mathrm{S}$ & $\mathrm{S}$ & $\mathrm{S}$ & $\mathrm{S}$ & $\mathrm{P}$ & $\mathrm{P}$ & $\mathrm{P}$ & $\mathrm{P}$ \\
\hline Santa Maria da Serra & $P$ & $\mathrm{~S}$ & $\mathrm{~S}$ & $\mathrm{~S}$ & $\mathrm{~S}$ & $\mathrm{~S}$ & $\mathrm{~S}$ & $\mathrm{~S}$ & $\mathrm{~S}$ & $\mathrm{P}$ & $\mathrm{P}$ & $\mathrm{P}$ \\
\hline Santa Mercedes & $\mathrm{N}$ & $\mathrm{N}$ & $\mathrm{P}$ & $\mathrm{S}$ & $\mathrm{S}$ & $\mathrm{S}$ & $\mathrm{S}$ & $\mathrm{P}$ & $\mathrm{N}$ & $\mathrm{N}$ & $\mathrm{N}$ & $\mathrm{N}$ \\
\hline S. Rita do Passa Quatro & $\mathrm{S}$ & $\mathrm{S}$ & NR & NR & NR & NR & $\mathrm{S}$ & $\mathrm{S}$ & $\mathrm{S}$ & $\mathrm{S}$ & $\mathrm{S}$ & $\mathrm{S}$ \\
\hline Santa Rita D'oeste & $\mathrm{N}$ & $\mathrm{N}$ & $\mathrm{P}$ & $\mathrm{S}$ & $\mathrm{S}$ & $\mathrm{S}$ & $\mathrm{P}$ & $\mathrm{N}$ & $\mathrm{N}$ & $\mathrm{N}$ & $\mathrm{N}$ & $\mathrm{N}$ \\
\hline Santa Rosa do Viterbo & $\mathrm{S}$ & $\mathrm{S}$ & NR & NR & NR & NR & $\mathrm{S}$ & $\mathrm{S}$ & $\mathrm{S}$ & $\mathrm{S}$ & $\mathrm{S}$ & $\mathrm{S}$ \\
\hline Santa Salete & $\mathrm{N}$ & $\mathrm{P}$ & $\mathrm{P}$ & $\mathrm{S}$ & $\mathrm{S}$ & $\mathrm{S}$ & $\mathrm{P}$ & $\mathrm{N}$ & $\mathrm{N}$ & $\mathrm{N}$ & $\mathrm{N}$ & $\mathrm{N}$ \\
\hline Santana da Ponte Pensa & $\mathrm{N}$ & $P$ & $\mathrm{P}$ & $\mathrm{S}$ & $\mathrm{S}$ & $\mathrm{S}$ & $\mathrm{P}$ & $\mathrm{N}$ & $\mathrm{N}$ & $\mathrm{N}$ & $\mathrm{N}$ & $\mathrm{N}$ \\
\hline Santana de Parnaíba & $\mathrm{S}$ & $\mathrm{S}$ & NR & NR & NR & NR & $\mathrm{S}$ & $\mathrm{S}$ & $\mathrm{S}$ & $\mathrm{S}$ & $\mathrm{P}$ & $\mathrm{P}$ \\
\hline Santo Anastácio & $\mathrm{P}$ & $\mathrm{P}$ & $\mathrm{S}$ & $\mathrm{S}$ & $\mathrm{S}$ & $\mathrm{S}$ & $\mathrm{S}$ & $\mathrm{S}$ & $\mathrm{P}$ & $\mathrm{P}$ & $\mathrm{P}$ & $\mathrm{N}$ \\
\hline Santo André & $\mathrm{S}$ & $\mathrm{S}$ & NR & NR & NR & NR & NR & $\mathrm{S}$ & $\mathrm{S}$ & $\mathrm{S}$ & $\mathrm{S}$ & $\mathrm{S}$ \\
\hline S. Antônio da Alegria & $\mathrm{S}$ & $\mathrm{S}$ & $\mathrm{S}$ & NR & NR & $\mathrm{S}$ & $\mathrm{S}$ & $\mathrm{S}$ & $\mathrm{S}$ & $\mathrm{S}$ & $\mathrm{S}$ & $\mathrm{S}$ \\
\hline S. Antônio de Posse & $\mathrm{P}$ & $\mathrm{S}$ & $\mathrm{S}$ & NR & NR & $\mathrm{S}$ & $\mathrm{S}$ & $\mathrm{S}$ & $\mathrm{S}$ & $\mathrm{S}$ & $\mathrm{P}$ & $\mathrm{P}$ \\
\hline $\begin{array}{l}\text { S. Antônio do } \\
\text { Aracanguá }\end{array}$ & $\mathrm{N}$ & $\mathrm{P}$ & $\mathrm{S}$ & $\mathrm{S}$ & $\mathrm{S}$ & $\mathrm{S}$ & $\mathrm{P}$ & $\mathrm{P}$ & $\mathrm{N}$ & $\mathrm{N}$ & $\mathrm{N}$ & $\mathrm{N}$ \\
\hline S. Antônio do Jardim & $\mathrm{S}$ & $\mathrm{S}$ & $\mathrm{S}$ & NR & NR & $\mathrm{S}$ & $\mathrm{S}$ & $\mathrm{S}$ & $\mathrm{S}$ & $\mathrm{S}$ & $\mathrm{P}$ & $\mathrm{P}$ \\
\hline S. Antônio do Pinhal & $\mathrm{S}$ & $\mathrm{NR}$ & NR & NR & NR & NR & NR & NR & $\mathrm{S}$ & $\mathrm{S}$ & $\mathrm{S}$ & $\mathrm{S}$ \\
\hline Santo Expedito & $\mathrm{N}$ & $\mathrm{P}$ & $\mathrm{S}$ & $\mathrm{S}$ & $\mathrm{S}$ & $\mathrm{S}$ & $\mathrm{S}$ & $\mathrm{P}$ & $\mathrm{N}$ & $\mathrm{N}$ & $\mathrm{N}$ & $\mathrm{N}$ \\
\hline Santópolis do Aguapeí & $\mathrm{P}$ & $\mathrm{P}$ & $\mathrm{S}$ & $\mathrm{S}$ & $\mathrm{S}$ & $\mathrm{S}$ & $\mathrm{S}$ & $\mathrm{P}$ & $\mathrm{P}$ & $\mathrm{P}$ & $\mathrm{N}$ & $\mathrm{N}$ \\
\hline Santos & $\mathrm{P}$ & $\mathrm{P}$ & $\mathrm{S}$ & $\mathrm{S}$ & $\mathrm{S}$ & $\mathrm{S}$ & $\mathrm{S}$ & $\mathrm{S}$ & $\mathrm{S}$ & $\mathrm{P}$ & $\mathrm{P}$ & $\mathrm{N}$ \\
\hline São Bento do Sapucai & $\mathrm{S}$ & $\mathrm{NR}$ & NR & NR & NR & NR & NR & NR & $\mathrm{S}$ & $\mathrm{S}$ & $\mathrm{S}$ & S \\
\hline S. Bernardo do Campo & $\mathrm{S}$ & $\mathrm{S}$ & NR & NR & NR & NR & NR & $\mathrm{S}$ & $\mathrm{S}$ & $\mathrm{S}$ & $\mathrm{S}$ & $\mathrm{S}$ \\
\hline São Caetano do Sul & $\mathrm{S}$ & $\mathrm{S}$ & NR & NR & NR & NR & NR & $\mathrm{S}$ & $\mathrm{S}$ & $\mathrm{S}$ & $\mathrm{S}$ & $\mathrm{S}$ \\
\hline São Carlos & $\mathrm{S}$ & NR & NR & NR & NR & NR & NR & NR & $\mathrm{S}$ & $\mathrm{S}$ & $\mathrm{S}$ & $\mathrm{S}$ \\
\hline São Francisco & $\mathrm{N}$ & $\mathrm{P}$ & $\mathrm{P}$ & $\mathrm{S}$ & $\mathrm{S}$ & $\mathrm{S}$ & $\mathrm{P}$ & $\mathrm{P}$ & $\mathrm{N}$ & $\mathrm{N}$ & $\mathrm{N}$ & $\mathrm{N}$ \\
\hline São João da Boa Vista & $\mathrm{S}$ & $\mathrm{S}$ & NR & NR & NR & NR & $\mathrm{S}$ & $\mathrm{S}$ & $\mathrm{S}$ & $\mathrm{S}$ & $\mathrm{S}$ & $\mathrm{S}$ \\
\hline S. João das Duas Pontes & $\mathrm{N}$ & $\mathrm{P}$ & $\mathrm{P}$ & $\mathrm{S}$ & $\mathrm{S}$ & $\mathrm{S}$ & $\mathrm{P}$ & $\mathrm{P}$ & $\mathrm{N}$ & $\mathrm{N}$ & $\mathrm{N}$ & $\mathrm{N}$ \\
\hline São João de Iracema & $\mathrm{N}$ & $\mathrm{P}$ & $\mathrm{P}$ & $\mathrm{S}$ & $\mathrm{S}$ & $\mathrm{S}$ & $\mathrm{P}$ & $\mathrm{P}$ & $\mathrm{N}$ & $\mathrm{N}$ & $\mathrm{N}$ & $\mathrm{N}$ \\
\hline São João do Pau D'alho & $\mathrm{N}$ & $\mathrm{N}$ & $\mathrm{P}$ & $\mathrm{S}$ & $\mathrm{S}$ & $\mathrm{S}$ & $\mathrm{S}$ & $\mathrm{P}$ & $\mathrm{N}$ & $\mathrm{N}$ & $\mathrm{N}$ & $\mathrm{N}$ \\
\hline São Joaquim da Barra & $\mathrm{P}$ & $\mathrm{P}$ & $\mathrm{S}$ & $\mathrm{S}$ & $\mathrm{S}$ & $\mathrm{S}$ & $S$ & $P$ & $\mathrm{P}$ & $\mathrm{P}$ & $P$ & $P$ \\
\hline São José da Bela Vista & $\mathrm{P}$ & $\mathrm{S}$ & $\mathrm{S}$ & $\mathrm{S}$ & $\mathrm{S}$ & $\mathrm{S}$ & $\mathrm{S}$ & $\mathrm{P}$ & $\mathrm{P}$ & $\mathrm{P}$ & $\mathrm{P}$ & $\mathrm{P}$ \\
\hline
\end{tabular}


Tabela 10. Genótipos de milho mais recomendados para cada início de ciclo nos municípios do Estado de São Paulo, durante os doze meses do ano. N: normal; P: precoce; S: superprecoce; NR: não recomendável

\begin{tabular}{|c|c|c|c|c|c|c|c|c|c|c|c|c|}
\hline \multirow{2}{*}{ Município } & \multicolumn{12}{|c|}{ Meses } \\
\hline & Jan & $\mathrm{Fev}$ & Mar & Abr & Mai & Jun & Jul & Ago & Set & Out & Nov & Dez \\
\hline São José do Barreiro & $\mathrm{P}$ & $\mathrm{S}$ & $\mathrm{S}$ & NR & NR & $\mathrm{S}$ & $\mathrm{S}$ & $\mathrm{S}$ & $\mathrm{S}$ & $\mathrm{P}$ & $\mathrm{P}$ & $\mathrm{P}$ \\
\hline São José do Rio Pardo & $\mathrm{S}$ & $\mathrm{S}$ & $\mathrm{S}$ & NR & NR & $\mathrm{S}$ & $\mathrm{S}$ & $\mathrm{S}$ & $\mathrm{S}$ & $\mathrm{S}$ & $\mathrm{P}$ & $\mathrm{P}$ \\
\hline São José do Rio Preto & $\mathrm{P}$ & $\mathrm{P}$ & $\mathrm{S}$ & $\mathrm{S}$ & $\mathrm{S}$ & $\mathrm{S}$ & $\mathrm{S}$ & $\mathrm{P}$ & $\mathrm{P}$ & $\mathrm{P}$ & $\mathrm{N}$ & $\mathrm{N}$ \\
\hline São José dos Campos & $\mathrm{P}$ & $\mathrm{S}$ & $\mathrm{S}$ & NR & NR & NR & $\mathrm{S}$ & $\mathrm{S}$ & $\mathrm{S}$ & $\mathrm{S}$ & $\mathrm{P}$ & $\mathrm{P}$ \\
\hline São Lourenço da Serra & $\mathrm{S}$ & $\mathrm{S}$ & NR & NR & NR & NR & NR & $\mathrm{S}$ & $\mathrm{S}$ & $\mathrm{S}$ & $\mathrm{S}$ & $\mathrm{S}$ \\
\hline São Luis do Paraitinga & $\mathrm{S}$ & NR & NR & NR & NR & NR & NR & $\mathrm{S}$ & $\mathrm{S}$ & $\mathrm{S}$ & $\mathrm{S}$ & $\mathrm{S}$ \\
\hline São Manuel & $\mathrm{S}$ & $\mathrm{S}$ & NR & NR & NR & NR & $\mathrm{S}$ & $\mathrm{S}$ & $\mathrm{S}$ & $\mathrm{S}$ & $\mathrm{S}$ & $\mathrm{S}$ \\
\hline São Miguel Arcanjo & $\mathrm{S}$ & $\mathrm{S}$ & NR & NR & NR & NR & NR & $\mathrm{S}$ & $\mathrm{S}$ & $\mathrm{S}$ & $\mathrm{S}$ & $\mathrm{S}$ \\
\hline São Paulo & $\mathrm{S}$ & $\mathrm{S}$ & NR & NR & NR & NR & NR & $\mathrm{S}$ & $\mathrm{S}$ & $\mathrm{S}$ & $\mathrm{S}$ & $\mathrm{S}$ \\
\hline São Pedro & $\mathrm{S}$ & $\mathrm{S}$ & $\mathrm{S}$ & NR & NR & NR & $\mathrm{S}$ & $\mathrm{S}$ & $\mathrm{S}$ & $\mathrm{S}$ & $\mathrm{P}$ & $\mathrm{P}$ \\
\hline São Pedro do Turvo & $\mathrm{P}$ & $\mathrm{S}$ & $\mathrm{S}$ & NR & NR & $\mathrm{S}$ & $\mathrm{S}$ & $\mathrm{S}$ & $\mathrm{S}$ & $\mathrm{P}$ & $\mathrm{P}$ & $\mathrm{P}$ \\
\hline São Roque & $\mathrm{S}$ & NR & NR & NR & NR & NR & NR & NR & $\mathrm{S}$ & $\mathrm{S}$ & $\mathrm{S}$ & $\mathrm{S}$ \\
\hline São Sebastiao & $\mathrm{N}$ & $\mathrm{N}$ & $\mathrm{P}$ & $\mathrm{S}$ & $\mathrm{S}$ & $\mathrm{S}$ & $\mathrm{S}$ & $\mathrm{P}$ & $\mathrm{N}$ & $\mathrm{N}$ & $\mathrm{N}$ & $\mathrm{N}$ \\
\hline São Sebastiao da Grama & $\mathrm{P}$ & $\mathrm{S}$ & $\mathrm{S}$ & $\mathrm{S}$ & $\mathrm{S}$ & $\mathrm{S}$ & $\mathrm{S}$ & $\mathrm{S}$ & $\mathrm{S}$ & $\mathrm{P}$ & $\mathrm{P}$ & $\mathrm{P}$ \\
\hline São Simão & $\mathrm{S}$ & $\mathrm{S}$ & $\mathrm{S}$ & $\mathrm{S}$ & $\mathrm{S}$ & $\mathrm{S}$ & $\mathrm{S}$ & $\mathrm{P}$ & $\mathrm{P}$ & $\mathrm{P}$ & $\mathrm{P}$ & $\mathrm{P}$ \\
\hline São Vicente & $\mathrm{N}$ & $\mathrm{N}$ & $\mathrm{P}$ & $\mathrm{S}$ & $\mathrm{S}$ & $\mathrm{S}$ & $\mathrm{S}$ & $\mathrm{P}$ & $\mathrm{N}$ & $\mathrm{N}$ & $\mathrm{N}$ & $\mathrm{N}$ \\
\hline Sarapuí & $\mathrm{S}$ & $\mathrm{S}$ & NR & NR & NR & NR & NR & $\mathrm{S}$ & $\mathrm{S}$ & $\mathrm{S}$ & $\mathrm{S}$ & $\mathrm{P}$ \\
\hline Sarutaia & $\mathrm{S}$ & $\mathrm{S}$ & NR & NR & NR & NR & NR & $\mathrm{S}$ & $\mathrm{S}$ & $\mathrm{S}$ & $\mathrm{S}$ & $\mathrm{S}$ \\
\hline Sebastianópolis do Sul & $\mathrm{P}$ & $\mathrm{P}$ & $\mathrm{S}$ & $\mathrm{S}$ & $\mathrm{S}$ & $\mathrm{S}$ & $\mathrm{S}$ & $\mathrm{P}$ & $\mathrm{P}$ & $\mathrm{N}$ & $\mathrm{N}$ & $\mathrm{N}$ \\
\hline Serra Azul & $\mathrm{S}$ & $\mathrm{S}$ & $\mathrm{S}$ & NR & NR & $\mathrm{S}$ & $\mathrm{S}$ & $\mathrm{S}$ & $\mathrm{S}$ & $\mathrm{S}$ & $\mathrm{P}$ & $\mathrm{P}$ \\
\hline Serra Negra & $\mathrm{S}$ & NR & NR & NR & NR & NR & NR & NR & $\mathrm{S}$ & $\mathrm{S}$ & $\mathrm{S}$ & $\mathrm{S}$ \\
\hline Serrana & $\mathrm{P}$ & $\mathrm{S}$ & $\mathrm{S}$ & $\mathrm{S}$ & $\mathrm{S}$ & $\mathrm{S}$ & $\mathrm{S}$ & $\mathrm{S}$ & $\mathrm{S}$ & $\mathrm{P}$ & $\mathrm{P}$ & $\mathrm{P}$ \\
\hline Sertãozinho & $\mathrm{P}$ & $\mathrm{S}$ & $\mathrm{S}$ & $\mathrm{S}$ & $\mathrm{S}$ & $\mathrm{S}$ & $\mathrm{S}$ & $\mathrm{S}$ & $\mathrm{P}$ & $\mathrm{P}$ & $\mathrm{P}$ & $\mathrm{P}$ \\
\hline Sete Barras & $\mathrm{N}$ & $\mathrm{N}$ & $\mathrm{P}$ & $\mathrm{S}$ & $\mathrm{S}$ & $\mathrm{S}$ & $\mathrm{S}$ & $\mathrm{P}$ & $\mathrm{P}$ & $\mathrm{N}$ & $\mathrm{N}$ & $\mathrm{N}$ \\
\hline Severinia & $\mathrm{P}$ & $\mathrm{S}$ & $\mathrm{S}$ & $\mathrm{S}$ & $\mathrm{S}$ & $\mathrm{S}$ & $\mathrm{S}$ & $\mathrm{S}$ & $\mathrm{P}$ & $\mathrm{P}$ & $\mathrm{P}$ & $\mathrm{P}$ \\
\hline Silveiras & $\mathrm{S}$ & $\mathrm{S}$ & $\mathrm{S}$ & NR & NR & NR & $\mathrm{S}$ & $\mathrm{S}$ & $\mathrm{S}$ & $\mathrm{S}$ & $\mathrm{P}$ & $\mathrm{P}$ \\
\hline Socorro & $\mathrm{S}$ & $\mathrm{S}$ & NR & NR & NR & NR & $\mathrm{S}$ & $\mathrm{S}$ & $\mathrm{S}$ & $\mathrm{S}$ & $\mathrm{S}$ & $\mathrm{S}$ \\
\hline Sorocaba & $\mathrm{P}$ & $\mathrm{S}$ & $\mathrm{S}$ & NR & NR & NR & $\mathrm{S}$ & $\mathrm{S}$ & $\mathrm{S}$ & $\mathrm{S}$ & $\mathrm{P}$ & $\mathrm{P}$ \\
\hline Sud Menucci & $\mathrm{N}$ & $\mathrm{P}$ & $\mathrm{S}$ & $\mathrm{S}$ & $\mathrm{S}$ & $\mathrm{S}$ & $\mathrm{S}$ & $\mathrm{P}$ & $\mathrm{N}$ & $\mathrm{N}$ & $\mathrm{N}$ & $\mathrm{N}$ \\
\hline Sumaré & $\mathrm{P}$ & $\mathrm{S}$ & $\mathrm{S}$ & NR & NR & NR & $\mathrm{S}$ & $\mathrm{S}$ & $\mathrm{S}$ & $\mathrm{S}$ & $\mathrm{P}$ & $\mathrm{P}$ \\
\hline Suzanápolis & $\mathrm{N}$ & $\mathrm{N}$ & $\mathrm{P}$ & $\mathrm{S}$ & $\mathrm{S}$ & $\mathrm{S}$ & $\mathrm{P}$ & $\mathrm{P}$ & $\mathrm{N}$ & $\mathrm{N}$ & $\mathrm{N}$ & $\mathrm{N}$ \\
\hline Suzano & $\mathrm{S}$ & $\mathrm{S}$ & NR & NR & NR & NR & NR & $\mathrm{S}$ & $\mathrm{S}$ & $\mathrm{S}$ & $\mathrm{S}$ & $\mathrm{S}$ \\
\hline Tabapuã & $\mathrm{P}$ & $\mathrm{P}$ & $\mathrm{S}$ & $\mathrm{S}$ & $\mathrm{S}$ & $\mathrm{S}$ & $\mathrm{S}$ & $\mathrm{P}$ & $\mathrm{P}$ & $\mathrm{P}$ & $\mathrm{P}$ & $\mathrm{P}$ \\
\hline Tabatinga & $\mathrm{P}$ & $\mathrm{P}$ & $\mathrm{S}$ & $\mathrm{S}$ & $\mathrm{S}$ & $\mathrm{S}$ & $\mathrm{S}$ & $\mathrm{P}$ & $\mathrm{P}$ & $\mathrm{P}$ & $\mathrm{P}$ & $\mathrm{N}$ \\
\hline Taboão da Serra & $\mathrm{S}$ & $\mathrm{S}$ & NR & NR & NR & NR & NR & $\mathrm{S}$ & $\mathrm{S}$ & $\mathrm{S}$ & $\mathrm{S}$ & $\mathrm{P}$ \\
\hline Taciba & $\mathrm{N}$ & $\mathrm{P}$ & $\mathrm{S}$ & $\mathrm{S}$ & $\mathrm{S}$ & $\mathrm{S}$ & $\mathrm{S}$ & $\mathrm{S}$ & $\mathrm{P}$ & $\mathrm{P}$ & $\mathrm{N}$ & $\mathrm{N}$ \\
\hline Taguaí & $\mathrm{P}$ & $\mathrm{S}$ & NR & NR & NR & NR & $\mathrm{S}$ & $\mathrm{S}$ & $\mathrm{S}$ & $\mathrm{S}$ & $\mathrm{P}$ & $\mathrm{P}$ \\
\hline Taiacu & $\mathrm{P}$ & $\mathrm{P}$ & $\mathrm{S}$ & $\mathrm{S}$ & $\mathrm{S}$ & $\mathrm{S}$ & $\mathrm{S}$ & $\mathrm{P}$ & $\mathrm{P}$ & $\mathrm{P}$ & $\mathrm{P}$ & $\mathrm{P}$ \\
\hline Taiúva & $\mathrm{P}$ & $\mathrm{S}$ & $\mathrm{S}$ & $\mathrm{S}$ & $\mathrm{S}$ & $\mathrm{S}$ & $\mathrm{S}$ & $\mathrm{P}$ & $\mathrm{P}$ & $\mathrm{P}$ & $\mathrm{P}$ & $\mathrm{P}$ \\
\hline Tambaú & $\mathrm{S}$ & $\mathrm{S}$ & NR & NR & NR & NR & $\mathrm{S}$ & $\mathrm{S}$ & $\mathrm{S}$ & $\mathrm{S}$ & $\mathrm{S}$ & S \\
\hline Tanabi & $\mathrm{P}$ & $\mathrm{P}$ & $\mathrm{S}$ & $\mathrm{S}$ & $\mathrm{S}$ & $\mathrm{S}$ & $\mathrm{S}$ & $\mathrm{P}$ & $\mathrm{P}$ & $\mathrm{P}$ & $\mathrm{P}$ & $\mathrm{P}$ \\
\hline Tapiraí & $\mathrm{S}$ & $\mathrm{S}$ & NR & NR & NR & NR & NR & $\mathrm{S}$ & $\mathrm{S}$ & $\mathrm{S}$ & $\mathrm{S}$ & $\mathrm{S}$ \\
\hline Tapiratiba & $\mathrm{S}$ & $\mathrm{S}$ & $\mathrm{S}$ & NR & NR & $\mathrm{S}$ & $\mathrm{S}$ & $\mathrm{S}$ & $\mathrm{S}$ & $\mathrm{S}$ & $\mathrm{S}$ & $\mathrm{S}$ \\
\hline
\end{tabular}


Tabela 10. Genótipos de milho mais recomendados para cada início de ciclo nos municípios do Estado de São Paulo, durante os doze meses do ano. N: normal; P: precoce; S: superprecoce; NR: não recomendável

\begin{tabular}{|c|c|c|c|c|c|c|c|c|c|c|c|c|}
\hline \multirow{2}{*}{ Município } & \multicolumn{12}{|c|}{ Meses } \\
\hline & Jan & Fev & Mar & Abr & Mai & Jun & Jul & Ago & Set & Out & Nov & Dez \\
\hline Taquaral & $\mathrm{P}$ & $\mathrm{S}$ & $\mathrm{S}$ & $\mathrm{S}$ & $\mathrm{S}$ & $\mathrm{S}$ & $\mathrm{S}$ & $\mathrm{P}$ & $\mathrm{P}$ & $\mathrm{P}$ & $\mathrm{P}$ & $\mathrm{P}$ \\
\hline Taquaritinga & $\mathrm{P}$ & $\mathrm{S}$ & $\mathrm{S}$ & $\mathrm{S}$ & $\mathrm{S}$ & $\mathrm{S}$ & $\mathrm{S}$ & $\mathrm{S}$ & $\mathrm{P}$ & $\mathrm{P}$ & $\mathrm{P}$ & $\mathrm{P}$ \\
\hline Taquarituba & $\mathrm{S}$ & $\mathrm{S}$ & NR & NR & NR & NR & $\mathrm{S}$ & $\mathrm{S}$ & $\mathrm{S}$ & $\mathrm{S}$ & $\mathrm{P}$ & $\mathrm{P}$ \\
\hline Taquarivaí & $\mathrm{S}$ & $\mathrm{S}$ & NR & NR & NR & NR & NR & $\mathrm{S}$ & $\mathrm{S}$ & $\mathrm{S}$ & $\mathrm{S}$ & $\mathrm{P}$ \\
\hline Tarabaí & $\mathrm{N}$ & $\mathrm{P}$ & $\mathrm{S}$ & $\mathrm{S}$ & $\mathrm{S}$ & S & $\mathrm{S}$ & $\mathrm{P}$ & $\mathrm{P}$ & $\mathrm{N}$ & $\mathrm{N}$ & $\mathrm{N}$ \\
\hline Taruma & $\mathrm{P}$ & $\mathrm{P}$ & $\mathrm{S}$ & $\mathrm{S}$ & $\mathrm{S}$ & $\mathrm{S}$ & $\mathrm{S}$ & $\mathrm{S}$ & $\mathrm{P}$ & $\mathrm{P}$ & $\mathrm{P}$ & $\mathrm{N}$ \\
\hline Tatuí & $\mathrm{P}$ & $\mathrm{S}$ & NR & NR & NR & NR & NR & $\mathrm{S}$ & $\mathrm{S}$ & $\mathrm{S}$ & $\mathrm{P}$ & $\mathrm{P}$ \\
\hline Taubaté & $\mathrm{P}$ & $\mathrm{S}$ & $\mathrm{S}$ & NR & NR & NR & $\mathrm{S}$ & $\mathrm{S}$ & $\mathrm{S}$ & $\mathrm{S}$ & $\mathrm{P}$ & $\mathrm{P}$ \\
\hline Tejupã & $\mathrm{S}$ & $\mathrm{S}$ & NR & NR & NR & NR & NR & $\mathrm{S}$ & $\mathrm{S}$ & $\mathrm{S}$ & $\mathrm{S}$ & $\mathrm{S}$ \\
\hline Teodoro Sampaio & $\mathrm{N}$ & $\mathrm{P}$ & $\mathrm{S}$ & $\mathrm{S}$ & $\mathrm{S}$ & $\mathrm{S}$ & $\mathrm{S}$ & $\mathrm{S}$ & $\mathrm{P}$ & $\mathrm{P}$ & $\mathrm{N}$ & $\mathrm{N}$ \\
\hline Terra Roxa & $\mathrm{P}$ & $\mathrm{P}$ & $\mathrm{S}$ & $\mathrm{S}$ & $\mathrm{S}$ & $\mathrm{S}$ & $\mathrm{S}$ & $\mathrm{P}$ & $\mathrm{P}$ & $\mathrm{P}$ & $\mathrm{P}$ & $\mathrm{P}$ \\
\hline Tietê & $P$ & $\mathrm{~S}$ & $\mathrm{~S}$ & NR & NR & NR & $\mathrm{S}$ & $\mathrm{S}$ & $\mathrm{S}$ & $\mathrm{S}$ & $\mathrm{P}$ & $\mathrm{P}$ \\
\hline Timburi & $\mathrm{P}$ & $\mathrm{S}$ & $\mathrm{S}$ & NR & NR & NR & $\mathrm{S}$ & $\mathrm{S}$ & $\mathrm{S}$ & $\mathrm{P}$ & $\mathrm{P}$ & $\mathrm{P}$ \\
\hline Torre de Pedra & $\mathrm{S}$ & $\mathrm{S}$ & NR & NR & NR & NR & NR & $\mathrm{S}$ & $\mathrm{S}$ & $\mathrm{S}$ & $\mathrm{S}$ & $\mathrm{S}$ \\
\hline Torrinha & $\mathrm{S}$ & $\mathrm{S}$ & NR & NR & NR & NR & NR & $\mathrm{S}$ & $\mathrm{S}$ & $\mathrm{S}$ & $\mathrm{S}$ & $\mathrm{S}$ \\
\hline Trabiju & $\mathrm{P}$ & $\mathrm{P}$ & $\mathrm{S}$ & $\mathrm{S}$ & $\mathrm{S}$ & S & $\mathrm{S}$ & $\mathrm{S}$ & $\mathrm{P}$ & $\mathrm{P}$ & $\mathrm{P}$ & $\mathrm{N}$ \\
\hline Tremembé & $\mathrm{S}$ & NR & NR & NR & NR & NR & NR & NR & $\mathrm{S}$ & $\mathrm{S}$ & $\mathrm{S}$ & $\mathrm{S}$ \\
\hline Três Fronteiras & $\mathrm{N}$ & $\mathrm{N}$ & $\mathrm{P}$ & $\mathrm{S}$ & $\mathrm{S}$ & $\mathrm{S}$ & $\mathrm{P}$ & $\mathrm{N}$ & $\mathrm{N}$ & $\mathrm{N}$ & $\mathrm{N}$ & $\mathrm{N}$ \\
\hline Tuiuti & $\mathrm{P}$ & $\mathrm{S}$ & $\mathrm{S}$ & NR & NR & $\mathrm{S}$ & $\mathrm{S}$ & $\mathrm{S}$ & $\mathrm{S}$ & $\mathrm{S}$ & $\mathrm{P}$ & $\mathrm{P}$ \\
\hline Tupã & $\mathrm{P}$ & $\mathrm{S}$ & $\mathrm{S}$ & $\mathrm{S}$ & $\mathrm{S}$ & $\mathrm{S}$ & $\mathrm{S}$ & $\mathrm{S}$ & $\mathrm{P}$ & $\mathrm{P}$ & $\mathrm{P}$ & $\mathrm{P}$ \\
\hline Tupi Paulista & $\mathrm{N}$ & $\mathrm{P}$ & $\mathrm{P}$ & $\mathrm{S}$ & $\mathrm{S}$ & $\mathrm{S}$ & $\mathrm{S}$ & $\mathrm{P}$ & $\mathrm{N}$ & $\mathrm{N}$ & $\mathrm{N}$ & $\mathrm{N}$ \\
\hline Turiuba & $\mathrm{N}$ & $\mathrm{P}$ & $\mathrm{S}$ & $\mathrm{S}$ & $\mathrm{S}$ & $\mathrm{S}$ & $\mathrm{S}$ & $\mathrm{P}$ & $\mathrm{N}$ & $\mathrm{N}$ & $\mathrm{N}$ & $\mathrm{N}$ \\
\hline Turmalina & $\mathrm{N}$ & $\mathrm{P}$ & $\mathrm{P}$ & $\mathrm{S}$ & $\mathrm{S}$ & $\mathrm{S}$ & $\mathrm{P}$ & $\mathrm{N}$ & $\mathrm{N}$ & $\mathrm{N}$ & $\mathrm{N}$ & $\mathrm{N}$ \\
\hline Ubarana & $\mathrm{N}$ & $\mathrm{P}$ & $\mathrm{S}$ & $\mathrm{S}$ & $\mathrm{S}$ & $\mathrm{S}$ & $\mathrm{S}$ & $\mathrm{P}$ & $\mathrm{P}$ & $\mathrm{N}$ & $\mathrm{N}$ & $\mathrm{N}$ \\
\hline Ubatuba & $\mathrm{P}$ & $\mathrm{S}$ & $\mathrm{S}$ & NR & NR & $\mathrm{S}$ & $\mathrm{S}$ & $\mathrm{S}$ & $\mathrm{S}$ & S & $\mathrm{P}$ & $\mathrm{P}$ \\
\hline Ubirajara & $\mathrm{P}$ & $\mathrm{S}$ & $\mathrm{S}$ & NR & NR & $\mathrm{S}$ & $\mathrm{S}$ & $\mathrm{S}$ & $\mathrm{S}$ & $\mathrm{P}$ & $\mathrm{P}$ & $\mathrm{P}$ \\
\hline Uchôa & $\mathrm{P}$ & $\mathrm{P}$ & $\mathrm{S}$ & $\mathrm{S}$ & $\mathrm{S}$ & $\mathrm{S}$ & $\mathrm{S}$ & $\mathrm{P}$ & $\mathrm{P}$ & $\mathrm{P}$ & $\mathrm{P}$ & $\mathrm{P}$ \\
\hline União Paulista & $\mathrm{N}$ & $\mathrm{P}$ & $\mathrm{S}$ & $\mathrm{S}$ & $\mathrm{S}$ & $\mathrm{S}$ & $\mathrm{S}$ & $\mathrm{P}$ & $\mathrm{P}$ & $\mathrm{N}$ & $\mathrm{N}$ & $\mathrm{N}$ \\
\hline Urânia & $\mathrm{N}$ & $\mathrm{P}$ & $\mathrm{S}$ & $\mathrm{S}$ & $\mathrm{S}$ & $\mathrm{S}$ & $\mathrm{S}$ & $\mathrm{P}$ & $\mathrm{N}$ & $\mathrm{N}$ & $\mathrm{N}$ & $\mathrm{N}$ \\
\hline Uru & $\mathrm{P}$ & $\mathrm{P}$ & $\mathrm{S}$ & $\mathrm{S}$ & $\mathrm{S}$ & $\mathrm{S}$ & $\mathrm{S}$ & $\mathrm{P}$ & $\mathrm{P}$ & $\mathrm{P}$ & $\mathrm{N}$ & $\mathrm{N}$ \\
\hline Urupês & $\mathrm{P}$ & $\mathrm{S}$ & $\mathrm{S}$ & $\mathrm{S}$ & $\mathrm{S}$ & $\mathrm{S}$ & $\mathrm{S}$ & $\mathrm{S}$ & $\mathrm{S}$ & $\mathrm{P}$ & $\mathrm{P}$ & $\mathrm{P}$ \\
\hline Valentim Gentil & $\mathrm{P}$ & $\mathrm{P}$ & $\mathrm{S}$ & $\mathrm{S}$ & $\mathrm{S}$ & $\mathrm{S}$ & $\mathrm{S}$ & $\mathrm{P}$ & $\mathrm{P}$ & $\mathrm{P}$ & $\mathrm{P}$ & $\mathrm{N}$ \\
\hline Valinhos & $\mathrm{P}$ & $\mathrm{S}$ & $\mathrm{S}$ & NR & NR & NR & $\mathrm{S}$ & $\mathrm{S}$ & $\mathrm{S}$ & $\mathrm{S}$ & $\mathrm{P}$ & $\mathrm{P}$ \\
\hline Valparaíso & $\mathrm{N}$ & $\mathrm{P}$ & $\mathrm{S}$ & $\mathrm{S}$ & $\mathrm{S}$ & $\mathrm{S}$ & $\mathrm{S}$ & $\mathrm{P}$ & $\mathrm{N}$ & $\mathrm{N}$ & $\mathrm{N}$ & $\mathrm{N}$ \\
\hline Vargem & $\mathrm{S}$ & NR & NR & NR & NR & NR & NR & $\mathrm{S}$ & $\mathrm{S}$ & $\mathrm{S}$ & $\mathrm{S}$ & S \\
\hline Vargem Grande do Sul & $\mathrm{S}$ & $\mathrm{S}$ & $\mathrm{S}$ & NR & NR & $\mathrm{S}$ & $\mathrm{S}$ & $\mathrm{S}$ & $\mathrm{S}$ & $\mathrm{S}$ & $\mathrm{P}$ & $\mathrm{P}$ \\
\hline $\begin{array}{ll}\text { Vargem } & \text { Grande } \\
\text { Paulista } & \end{array}$ & $\mathrm{P}$ & $\mathrm{S}$ & $\mathrm{S}$ & NR & NR & NR & $\mathrm{S}$ & $\mathrm{S}$ & $\mathrm{S}$ & $\mathrm{S}$ & $\mathrm{P}$ & $\mathrm{P}$ \\
\hline Várzea Paulista & $\mathrm{S}$ & $\mathrm{S}$ & NR & NR & NR & NR & $\mathrm{S}$ & $\mathrm{S}$ & $\mathrm{S}$ & $\mathrm{S}$ & $\mathrm{S}$ & $\mathrm{P}$ \\
\hline Vera Cruz & $\mathrm{S}$ & $\mathrm{S}$ & $\mathrm{S}$ & NR & NR & NR & $\mathrm{S}$ & $\mathrm{S}$ & $\mathrm{S}$ & $\mathrm{S}$ & $\mathrm{P}$ & $\mathrm{P}$ \\
\hline Vinhedo & $\mathrm{S}$ & $\mathrm{S}$ & NR & NR & NR & NR & NR & $\mathrm{S}$ & $\mathrm{S}$ & $\mathrm{S}$ & $\mathrm{S}$ & $\mathrm{S}$ \\
\hline Viradouro & $\mathrm{P}$ & $\mathrm{S}$ & $\mathrm{S}$ & $\mathrm{S}$ & $\mathrm{S}$ & $\mathrm{S}$ & $\mathrm{S}$ & $\mathrm{P}$ & $\mathrm{P}$ & $\mathrm{P}$ & $\mathrm{P}$ & $\mathrm{P}$ \\
\hline Vista Alegre do Alto & $\mathrm{P}$ & $\mathrm{P}$ & $\mathrm{S}$ & $\mathrm{S}$ & $\mathrm{S}$ & $\mathrm{S}$ & $\mathrm{S}$ & $\mathrm{P}$ & $\mathrm{P}$ & $\mathrm{P}$ & $\mathrm{P}$ & $\mathrm{P}$ \\
\hline
\end{tabular}


Tabela 10. Genótipos de milho mais recomendados para cada início de ciclo nos municípios do Estado de São Paulo, durante os doze meses do ano. N: normal; P: precoce; S: superprecoce; NR: não recomendável

\begin{tabular}{lcccccccccccc}
\hline \multicolumn{1}{c}{ Município } & \multicolumn{1}{c}{ Jan } & Fev & Mar & Abr & Mai & Jun & Jul & Ago & Set & Out & Nov & Dez \\
\hline Vitória Brasil & $\mathrm{N}$ & $\mathrm{P}$ & $\mathrm{P}$ & $\mathrm{S}$ & $\mathrm{S}$ & $\mathrm{S}$ & $\mathrm{P}$ & $\mathrm{N}$ & $\mathrm{N}$ & $\mathrm{N}$ & $\mathrm{N}$ & $\mathrm{N}$ \\
Votorantim & $\mathrm{P}$ & $\mathrm{S}$ & $\mathrm{NR}$ & $\mathrm{NR}$ & $\mathrm{NR}$ & $\mathrm{NR}$ & $\mathrm{S}$ & $\mathrm{S}$ & $\mathrm{S}$ & $\mathrm{S}$ & $\mathrm{P}$ & $\mathrm{P}$ \\
Votuporanga & $\mathrm{P}$ & $\mathrm{P}$ & $\mathrm{S}$ & $\mathrm{S}$ & $\mathrm{S}$ & $\mathrm{S}$ & $\mathrm{S}$ & $\mathrm{P}$ & $\mathrm{P}$ & $\mathrm{P}$ & $\mathrm{P}$ & $\mathrm{N}$ \\
Zacarias & $\mathrm{N}$ & $\mathrm{P}$ & $\mathrm{S}$ & $\mathrm{S}$ & $\mathrm{S}$ & $\mathrm{S}$ & $\mathrm{S}$ & $\mathrm{P}$ & $\mathrm{P}$ & $\mathrm{N}$ & $\mathrm{N}$ & $\mathrm{N}$ \\
\hline
\end{tabular}

\subsection{Considerações finais}

Os resultados obtidos no presente trabalho devem ser utilizados apenas como um indicativo da variação espaço-temporal das variáveis envolvidas. A tomada de decisão deve ser orientada pela analise econômica de todos os fatores que interferem no processo produtivo, principalmente no que diz respeito à época de semeadura e a adoção ou não da prática de irrigação.

A definição do ciclo em função da época do ano, conforme adotado no presente trabalho (Tabela 10), parece ser válida como critério de recomendação ou não dos genótipos mais adequados em cada localidade, haja vista que os ciclos normais predominaram nas épocas mais quentes do ano, enquanto nas épocas e/ou locais mais frios houve predominância de ciclos superprecoces ou não se recomenda nenhum dos genótipos. Como exemplo pode-se mencionar o município de Campos do Jordão, que apresentou limitação para o cultivo de milho, não sendo recomendada a exploração desta cultura em nenhuma época do ano.

Com o aumento da competitividade nos diversos setores da economia, o desenvolvimento de cinturões de produção de uma determinada cultura, em regiões mais favoráveis, onde ela possa mais facilmente expressar o seu potencial produtivo, é extremamente importante. 


\section{CONCLUSÕES}

De acordo com os resultados obtidos pode-se concluir que: (i) o modelo proposto é utilizável para definir a ordem de grandeza da produtividade potencial de grãos de milho no Estado de São Paulo; (ii) o modelo proposto pode ser utilizado como ferramenta para o planejamento regional da implantação da cultura de milho, possibilitando a identificação dos locais e das épocas do ano mais indicados para o cultivo de milho; (iii) as épocas do ano com maiores valores de temperatura e radiação solar resultaram em aumento da produtividade potencial de grãos de milho; (iv) a deficiência hídrica ocorrida no período de junho a setembro causou redução considerável na produtividade de grãos de milho, especialmente na região Noroeste do Estado de São Paulo. 


\section{REFERÊNCIAS BIBLIOGRÁFICAS}

ADDISCOTT, T.M. Simulation modelling and soil behaviour. Geoderma, v.69, p.1540, 1993.

ALFONSI, R.R.; PEDRO JÚNIOR., M.J.; CAMARGO, M.B.P. et al. Zoneamento agroclimático e probabilidade de atendimento hídrico para as culturas de soja, milho, arroz de sequeiro e feijão no estado de São Paulo. Campinas: Instituto Agronômico, 1995. 8p. (Boletim Científico).

ALFONSI, R.R.; VICTORIA FILHO, R.; SENTELHAS, P.C. Épocas de semeadura para a cultura do milho no Estado de São Paulo, baseadas na probabilidade de atendimento hídrico. Revista Brasileira de Agrometeorologia, v.5, n.1, p.43-49, 1997.

ASSIS, F.N.; MENDEZ, M.E.G. Relação entre radiação fotossinteticamente ativa e radiação global. Pesquisa Agropecuária Brasileira, v.2, n.7, p.797-800, 1989.

BARROS, A.H.C. Análise de crescimento, do desenvolvimento e da produtividade da cultura do milho (Zea mays L.): Experimentos e modelos. Viçosa, 1998. 85p. Dissertação (Mestrado) - Universidade Federal de Viçosa.

BASANTA, M.D.V. Modelo para estimativa do volume máximo de calda visando a aplicação foliar de produtos químicos na cultura de milho (Zea mays L.). Piracicaba, 1999. 65 p. Dissertação (Mestrado) - Escola Superior de Agricultura "Luiz de Queiroz", Universidade de São Paulo.

CAMARGO, A.P. Contribuição para a determinação da evapotranspiração potencial no Estado de São Paulo. Bragantia, v.21, p.163-203, 1962. 
CAMARGO, A.P.; SENTELHAS, P.C. Avaliação de modelos para a estimativa de evapotranspiração potencial mensal em base diária, para Campinas e Ribeirão Preto (SP). In: CONGRESSO BRASILEIRO DE AGROMETEOROLOGIA. 9., Campina Grande, 1995. Anais Campina Grande: SBA, 1995. p.415-417.

CAMARGO, M.B.P.; ARRUDA, H.V.; PEDRO JR., M.J. et al. Melhores épocas de plantio do trigo no Estado de São Paulo baseadas na probabilidade de atendimento hídrico. Bragantia, v.44, n.1, p.255-261, 1985.

CASTRO, P.T. Evapotranspiração atual e potencial de uma cultura de milho (Zea mays L.). Piracicaba, 1979. 61p. Dissertação (Mestrado) - Escola Superior de Agricultura “Luiz de Queiroz”, Universidade de São Paulo.

CERRI, C.E.P. Variabilidade espacial e temporal do carbono do solo na conversão de floresta em pastagens na Amazônia Ocidental (Rondônia). Piracicaba, 2003. 159p. Tese (Doutorado) - Escola Superior de Agricultura "Luiz de Queiroz", Universidade de São Paulo.

COELHO, A.M.; CRUZ, J.C.; PEREIRA FILHO, I.A. Rendimento de milho no Brasil: chegamos ao máximo? Piracicaba: Potafos, 2003. 12p. (Informações agronômicas, 101)

COMPANHIA NACIONAL DE ABASTECIMENTO. Avaliação da safra agrícola 2002/2003: sexto levantamento, agosto/2003. http://www.conab.gov.br (19 fev 2004a).

COMPANHIA NACIONAL DE ABASTECIMENTO. Comparativo da área, produção e produtividade safra 2001/2002 e safra 2002/2003. http://www.conab.gov.br (18 fev 2004b).

COSTA, A.F.S. Influência das condições climáticas no crescimento e desenvolvimento de plantas de milho (Zea mays L), avaliada em diferentes épocas de plantio. Viçosa, 1994. 109p. Dissertação (Doutorado) - Universidade Federal de Viçosa.

CRUZ, J.C.; CORREA, L.A.; SANS, L.M.A. et al. Avaliação de cultivares de milho safrinha na região Centro-Sul. In: SEMINARIO SOBRE A CULTURA DO MILHO SAFRINHA, 2., Assis. 1994. Resumos Campinas: Instituto Agronômico, 1994. p.35-40. 
DAKER, A. A água na agricultura: manual de hidráulica agrícola. 3.ed. Rio de Janeiro: Freitas Bastos, 1969. v.3. 453p.

De WIT, C.T. Photosynthesis of leaf canopies. Wageningen: Pudoc, 1965. 57p (Agriculture Research Report, 663)

De WIT, C.T. Simulation of living systems. In: PENNING DE VRIES, F.W.T.; LAAR, H.H.van (Ed.). Simulation of plant growth and crops production. Wageningem: Pudoc, 1982. p.3-8.

DEEGLE, P.J.; RIBEIRO JÚNIOR, P.J. Model based geostatistics. In: SIMPÓSIO NACIONAL DE PROBABILIDADE E ESTATÍSTICA, 14., Caxambu, 2000. Publicação especial. São Paulo: ABE, 2000. 129p.

DOORENBOS, J.; KASSAM, A.H. Efeito da água no rendimento das culturas. Trad. de GHEYI, H. Campina Grande: UFPB, 1994. 306p. (Estudos FAO: Irrigação e Drenagem, 33)

DOORENBOS, J.; PRUITT, W.O. Necessidades hídricas das culturas. Trad. de GHEYI, H. Campina Grande: UFPB, 1997. 204p. (Estudos FAO: Irrigação e Drenagem, 24)

DOURADO NETO, D. Modelos fitotécnicos referentes à cultura do milho. Piracicaba, 1999. 229p. Tese (Livre-Docência) - Escola Superior de Agricultura "Luiz de Queiroz”, Universidade de São Paulo.

DOURADO NETO, D.; SAAD, A.M.; JONG van LIER, Q. Curso de agricultura irrigada. Piracicaba: ESALQ, Depto. de Agricultura, 1991. 190p.

EMPRESA BRASILEIRA DE PESQUISA AGROPECUÁRIA. Cultivares de milho disponíveis no mercado de sementes do Brasil para a safra 2003/04. http://www.cnpms.embrapa.br/cultivares/index.html (28 jan. 2004).

FANCELLI, A.L.; LIMA, U.A. Milho: Produção, pré-processamento e transformação agroindustrial. São Paulo: FEALQ, 1982. 112p.

FANCELli, A.L.; DOURADO NETO, D. Produção de milho. Guaíba: Agropecuária, 2000. 360p.

FLOSS, E.L. Fotossíntese das plantas cultivadas. Passo Fundo: Universidade de Passo Fundo, 2002. 135p. (Cadernos de agronomia, 5) 
FORSTHOFER, E.L., SILVA, P.R.F. da, ARGENTA, G. et al. Fenologia, crescimento e desenvolvimento de híbridos de molho em três épocas de semeadura. Congresso Nacinal de Milho e Sorgo. 24. CD-ROM. Resumos expandidos. Florianópolis. ABMS/Embrapa Milho e Sorgo/Epagri. 2002.

FRIZZONE, J.A.; RETTORE, P.R.; PERERIRA, G.T. Análise de distribuição das precipitações em períodos de 5 a 10 dias na região de pereira Barreto (SP) utilizando a distribuição gama incompleta. Irrigação e Tecnologia Moderna, v.22, p.2-4, 1985.

FROGBROOK, Z.L.; OLIVER, M.A.; SALAHI, M.; ELLIS, R.H. Exploring the spatial relations between cereal yield and soil chemical properties and the implications for sampling. Soil Use and Management, v.18, p.1-9, 2002.

GADIOLI, J.L. Estimativa de rendimento de grãos e caracterização fitotécnica da cultura de milho (Zea mays L). Piracicaba, 1999. 86p. Dissertação (Mestrado.) Escola Superior de Agricultura “Luiz de Queiroz”, Universidade de São Paulo.

GASTON, L.A.; LOCKE, M.A.; ZABLOTOWICS, R.M.; REDDY, K.N. Spatial variability of soil properties and weed population in the Mississippi delta. Soil Science of America Jounal, v.65, p.449-459, 2001.

GOMES, F.P.; GARCIA, C.H. Estatística aplicada a experimentos agronômicos e florestais: exposição com exemplos e orientações para uso de aplicativos. Piracicaba: FEALQ, 2002. 309p.

GONÇALVES, A.C.A. Variabilidade espacial de propriedades físicas do solo para fins de manejo da irrigação. Piracicaba, 1997. 118p. Tese (Doutorado) - Escola Superior de Agricultura “Luiz de Queiroz", Universidade de São Paulo.

GOOVAERTS, P. Geostatistes in soil science: state-of-the-art and perspectives. Geoderma, v.89, n.1/2, p.1-45, 1999.

GOUDRIAAN, J.; LAAR, H.H. van. Modelling potential crop growth processes: the textbooh with exercises. Dordrecht: Kluwer, 1992. 239p. 
HEEMST, H.D.J. van. Physiological principles. In: KEULEN, H.van.; WOLF, J. Modeling of agricultural production: Weather, soils and crops. Wageningem: Pudoc, 1986. p.13-26.

HOOGENBOOM, G. Contribution of agrometeorology to the simulation of crop production and its applications. Agricultural and Forest Meteorology, n.103, 2000. p.137-157.

KEULEN, H.van.; WOLF, J. Modeling of agricultural production: Weather, soils and crops. Wageningem: Pudoc, 1986. 463p.

KEULEN, H.van.; PENNING DE VRIES, F.W.T.; DRESS, E.M. A summary model for crop growth. In: PENNING DE VRIES, F.W.T.; VAN LAAR, H. H. (Ed). Simulation of plant growth and crop production. Wageningem: Pudoc, 1982. p.87-97.

KUBINS, S. Measurement of radiant energy. In: SESTAK, Z.; CATSKY, J.; JARVIS, P.G. Plant photosynthetic production, manual of methods. Hague: W. Junk 1971. p.702-765.

LEAL, M.A.A.; DE-POLLI, H. Aplicações de modelos ao estudo da matéria orgânica do solo. In: SANTOS, G.A.; CAMARGO, F.A.O. (Ed.). Fundamentos da matéria orgânica do solo: ecossistemas tropicais e subtropicais. Porto Alegre: Gênesis, 1999. p.245-264.

LIMA, M.G. Calibração e validação do modelo ceres-maize em condições tropicais do BrasPiracicaba, 1995. 119p. Tese (Doutorado) - Escola Superior de Agricultura “Luiz de Queiroz”, Universidade de São Paulo.

LOZARDA, B.I.; ANGELOCI, L.R. Efeito da temperatura do ar e da disponibilidade hídrica do solo na duração de subperíodos e na produtividade de um híbrido de milho (Zea mays, L.). Revista Brasileira de Agrometeorologia, v.7, n.1, p.37-43, 1999.

MATHERON, G. The theory of regionalized variables and its applications. Fointanebleu: Cahiers du Centre de Morphologie Mathématique de Fointanebleu, 1971. $211 \mathrm{p}$. 
MATZENAUER, R.; BERGAMASCHI, H.; BERLATO, M.A.; RIBOLDI, J. Relações entre rendimento de milho e variáveis hídricas. Revista Brasileira de Agrometeorologia, v.3, p.85-92, 1995.

MEDEIROS, S.L.P.; WESTPHALEN, S.L.; MATZENAUER, R.; BERGAMASCHI, H. Relações entre evapotranspiração e rendimento de grãos de milho. Pesquisa Agropecuária Brasileira, v.26, n.1, p.1-10, 1991.

MONDRAGÓN, V.E.C. Estimativa da produtividade da cultura do milho em minas gerais, baseada em vaiáveis climáticas e em tendência tecnológica. Viçosa, 1990. 68p. Dissertação (Mestrado) - Universidade Federal de Viçosa.

MUCHOV, R.C.; HAMMER, G.L.; CARBERRY, P.S. Optimizing crop and cultivar selection in response to climatic risk. In: MUCHOV, R.C.; BELLAMY, J.A. (Ed.) Climatic risk in crop production models and management for the semiarid Tropics and Subtropics. Wallingford: CAB International, 1991. p.235-262.

OLIVER, M.A.; WEBSTER, R. How geostatistic can help you. Soil Use and Management, v.7, n.4, p.206-217, 1991.

OLIVEIRA, R.F. de. Fotossíntese. Piracicaba: Depto. de Botânica/ESALQ, 1990. 35p.

OMETTO, J.C. Bioclimatologia vegetal. São Paulo: Ceres, 1981. 440p.

PANDOLFO, C. Parâmetros básicos para uso na modelagem do rendimento de matéria seca em alfafa (Medicago sativa L.). Porto Alegre, 1995. 128p. Dissertação (Mestrado) - Faculdade de Agronomia, Universidade do Rio Grande do Sul.

PATERNIANI, E. Melhoramento e produção de milho no BrasSão Paulo: Fundação Cargill, 1978. 650p.

PAUSTIAN, K.; PARTON, W.J.; PERSSON, J. Modeling soil organic matter in organic-amended and nitrogen fertilized long-term plots. Soil Science Society of America Journal, v.56, p.476-488, 1992.

PEDRO JÚNIOR., M.J.; ALFONSI, R.R.; CAMARGO, M.B.P. et al. Disponibilidade de radiação solar global para o estado de São Paulo. Campinas, Instituto Agronômico, 1989. 13p. (Boletim Técnico, 123) 
PEREIRA, A.R.; MACHADO, E.C. Análise quantitativa do crescimento de comunidades vegetais. Campinas, Instituto Agronômico, 1987. 33p. (Boletim Técnico, 114)

PEREIRA, A.R.; ANGELOCCI, L.R.; SENTELHAS, P.C. Agrometeorologia: fundamentos e aplicações práticas. Guaíba: Agropecuária, 2002. 478p.

PIONEER. Resultados Milho: safra 2001/2002. Santa Cruz do Sul: Pioneer Sementes, 2002. 20p.

RAMALHO FILHO, A.; BEEK, K.J. Sistema de avaliação da aptidão agrícola das terras. Rio de Janeiro: EMBRAPA-CNPS, 1995. 65p.

REICHARDT, K. A água em sistemas agrícolas. São Paulo: Manole, 1987. 188p.

REICHARDT, K.; TIMM, L.C. Solo, planta e atmosfera: Conceitos, processos e aplicações. Barueri: Manole, 2004. 478p.

REYNOLDS, J.F. Some misconceptions of mathematical modeling. What's New Plant Physiology, v.10, n.11, p.41-44, 1979.

ROSENBERG, N.J.; BLAD, B.L.; VERMA, S.B. Microclimate: The biological environment. New York: Jonh Wiley, 1983. 495p.

SÁ, M. de. Aspectos morfológicos e fisiológicos de cultivares modernas e primitivas de milho. Lavras, 2001. 54p. Dissertação (Mestrado) - Universidade Federal de Lavras.

SALISBURY, F.B. Plant Physiology. Belmont: Wadsworth, 1992. 682p.

SAUNDERS, L.C.U.; CASTRO, P.T.; MATIAS FILHO, J.; BEZERRA, F.M.L. Uso consutivo da cultura do milho sob condições naturais de precipitação na microrregião homogênea de Quixeramobim. In: CONGRESSO BRASILEIRO DE ENGENHARIA AGRÍCOLA, 14., Fortaleza, 1984. Anais. Fortaleza: UFC/SBEA, 1984. p.142-151.

SOLER, C.T. Uso do modelo CERES-Maize para identificação de características genéticas desejáveis para milho "Safrinha" e determinação de práticas adequadas de manejo em condições de risco climático. 2000. 86p. Dissertação (Mestrado) Escola Superior de Agricultura “Luiz de Queiroz”, Universidade de São Paulo. 
SPITTERS, C.J.T.; TOUSSAINT, H.A.J.; GOUDRIAAN, J. Separating the diffuse and direct component of global radiation and its implications for modelling canopy photosynthesis. I: Components of incoming solar radation. Agricultural and Forest Meteorology, n.38, p.217-229, 1986a.

SPITTERS, C.J.T.; TOUSSAINT, H.A.J.; GOUDRIAAN, J. Separating the diffuse and direct component of global radiation and its implications for modelling canopy photosynthesis. II: Calculation of canopy photosynthesis. Agricultural and Forest Meteorology, n.38, p.231-242, 1986 b.

THOM, H.C.S. Some methods of climatological analysis. Roma: FAO, 1966. 50p. (FAO. Technical Notes 81)

THORNLEY, J.H.M. Mathematical models in plant phisiology: a quantitative approach to problems in plant crop phisiology. London: Academic Press, 1976. $318 \mathrm{p}$.

THORNTHWAITE, C.W. An approach toward a rational classification of climate. Geogr. Review, n.38, p. 55-94, 1948.

THORNTHWAITE, C.W.; MATHER, J.R. The water balance. Publications in Climatology. New Jersey: Drexel Institute of Technology, 1955. 104p.

VANCLOOSTER, M.; VIAENE, J.; DIELS, J.; CHRISTIAENS, K. Wave: a mathematical model for simulating water and agrochemicals in the soil and vadose environment. Leuven: Katholieke Universiteit Leuven Press, 1994. 1v.

VIANA DA MATA, J.D.; GONÇALVES, A.C.A.; VIEIRA, S.R.; FOLEGATTI, M.V. Relação entre produtividade e resistência à penetração em área irrigada por pivô central, sob dois sistemas de preparo. Acta Scientriarum, v.21, n.3, p.519-525, 1999.

VILLA NOVA, N.A.; SANTIAGO, A.V.; RESENDE, F.C. Energia solar: aspectos físicos e de captura pela biomassa. Piracicaba: ESALQ/USP, 2001. 20p.

VILLA NOVA, N.A.; PEDRO JÚNIOR, M.; PEREIRA, A.R.; OMETTO, J.C. Estimativa de graus-dia acumulados acima de qualquer temperatura base, em função das temperaturas máxima e mínima. São Paulo: Instituto de Geografia/USP, 1972. 8p.(Cadernos de Ciências da Terra, 30) 
VILLANUEVA, J.G. Estudo do coeficiente de transferência do vapor d'água sobre a cultura do feijão. Piracicaba, 1987. 58p. Dissertação (Mestrado) - Escola Superior de Agricultura "Luiz de Queiroz", Universidade de São Paulo.

VYN, T.J. Nutrient placement and high yield management in corn. In: CONFERENCE PROCEEDINGS, Indianapolis, p.7-9, 2001.

YAMADA, T. O nitrogênio e o potássio na adubação da cultura do milho. Piracicaba: Potafos, 1997. 4p. ( Informações Agronômicas, 78)

WEBSTER, R. Quantitative spatial analysis in the field. Advances in Soil Science, v.3, p.1-70, 1985. 